Luigi Ambrosio $\cdot$ Vicent Caselles · Simon Masnou $\cdot$ Jean-Michel Morel

\title{
Connected components of sets of finite perimeter and applications to image processing
}

Received July 27, 1999 / final version received June 8, 2000

Published online November 8, 2000 - (c) Springer-Verlag \& EMS 2000

\begin{abstract}
This paper contains a systematic analysis of a natural measure theoretic notion of connectedness for sets of finite perimeter in $\mathbb{R}^{\mathrm{N}}$, introduced by $\mathrm{H}$. Federer in the more general framework of the theory of currents. We provide a new and simpler proof of the existence and uniqueness of the decomposition into the so-called $M$-connected components. Moreover, we study carefully the structure of the essential boundary of these components and give in particular a reconstruction formula of a set of finite perimeter from the family of the boundaries of its components. In the two dimensional case we show that this notion of connectedness is comparable with the topological one, modulo the choice of a suitable representative in the equivalence class. Our strong motivation for this study is a mathematical justification of all those operations in image processing that involve connectedness and boundaries. As an application, we use this weak notion of connectedness to provide a rigorous mathematical basis to a large class of denoising filters acting on connected components of level sets. We introduce a natural domain for these filters, the space $\operatorname{WBV}(\Omega)$ of functions of weakly bounded variation in $\Omega$, and show that these filters are also well behaved in the classical Sobolev and BV spaces.
\end{abstract}

\section{Introduction}

Recently, and from different points of view, there has been a renewed interest in measure theoretic notions of connectedness [21,71] (see also [36]). For the case of BV functions and sets of finite perimeter, we shall present here a theory as much complete as possible, giving at the same time new and simpler proofs of some classical results. We are strongly motivated by the use of such objects as "connected components of level sets", "Jordan curves", etc. in digital image technology. One of our aims will be to give a well founded mathematical model

L. Ambrosio: Scuola Normale Superiore, Piazza dei Cavalieri 7, 56126 Pisa, Italy, e-mail: luigi@ambrosio.sns.it

V. Caselles: Departament de Tecnologia, Universitat Pompeu-Fabra, Passeig de Circumvalacio 8, 08003 Barcelona, Spain, e-mail: vicent.caselles@tecn.upf.es

S. Masnou: Analyse Numérique, BC 187, Université Pierre et Marie Curie, 75252 Paris Cedex 05, France, e-mail: masnou@ann.jussieu.fr

J.-M. Morel: CMLA, ENS Cachan, 61 Av. du Président Wilson, 94235 Cachan, France, e-mail: Jean-Michel.Morel@cmla.ens-cachan.fr 
for the well-spread use, in image processing and image analysis, of connectedness properties to create regions or "shapes" in an image. Also, the description of the regions boundaries in terms of "curves" and the existence of "level lines" in an image will be justified.

\section{The extraction of shapes from images}

Image analysis theory admits the existence of "shapes" in an image. There are many theories and algorithms for the extraction of such objects from a digital image. Some theories propose a segmentation of the image into connected regions by a variational principle [52,53]. Other theories assume that the discontinuity set of the image provides curves which, in some way or another, can be closed by an algorithm (see [8,50] and the discussion in [7]). Canny's filter [9], for instance, computes a set of discontinuity points in the image which must be thereafter connected by some variational principle. The obtained curves are supposed to be the boundaries of the "shapes" of the image. Many pattern recognition theories directly assume the existence of Jordan curves in the image (without explaining how such shapes should be extracted) and focus on subsequent recognition algorithms [33,40,41].

To summarize, most shape analysis methods deal with connected regions and their surrounding curves, and the curves surrounding their holes as well. Now, the ways such regions and curves are extracted are rather diverse and uncertain. Indeed, this extraction is often based on "edge detection theory", a wide galaxy of heuristic algorithms finding boundaries in an image. See [14] for a survey of these techniques and also the book [51] for an attempt of mathematical classification. We shall see, however, that in most practical cases shapes can and should be extracted as connected components of level sets of the image, and Jordan curves as their boundaries.

\section{Why scalar images and not vector (colour) images?}

Let us first define the digital image as raw object. We shall then discuss what the alternatives for the extraction of shapes are. An image can be realistically modelled as a real function $u(x)$ where $x$ represents an arbitrary point of $\mathbb{R}^{\mathrm{N}}(N=2$ for usual snapshots, 3 for medical images or movies, 4 for moving medical images) and $u(x)$ denotes the grey level, or colour, at $x$. In general, the image domain is finite (a hyperrectangle) but there will be no loss of generality in assuming that it is defined on the whole euclidean space. An image may be panchromatic; in that case $u(x)$ represents the photonic flux over a wide band of wavelengths and we have a proper grey level image. Now, $u(x)$ may also represent a colour intensity, when the photonic flux is subjected to a colour selective filter. In the following, we always consider scalar images, that is, images with a single channel, be it colour or grey level. When several channels have been captured simultaneously, we obtain naturally vector images, with e.g. three channels (Red, Green, Blue). It may appear at first as a restriction not to consider vector images, but only scalar ones. Indeed, the use of colour images is well-spread in human communication 
and most image processing and analysis operators must therefore be defined on vector images. Now, the redundancy of the colour images (from the perceptual viewpoint) is high. It is well admitted that the essential geometric features of any natural image are contained in its panchromatic (grey level) representation. Given a colour image, this panchromatic version is simply given as a linear positive combination of the three colour channels. As a consequence of this empiric observation, most image processing operators are defined separately for each channel and most image analysis operators are expected to give essentially the same result no matter whether applied to each one of the colour channel or to the panchromatic (grey level) version of the image. This fact, that geometric information essentially be contained in the grey level representation, can be checked by numerical experimental procedures [11]. These procedures involve discrete implementations of operators computing connected components of level sets, so that they are part of our motivations for investigating connectedness.

\section{Image formation}

From now on, and for the reasons just developed, we shall limit ourselves to the problem of connectedness in scalar images. We sketch in the following some aspects of image formation which will be relevant to our discussion. The process of image formation is, in a first approximation, given by the following formula [70]:

$$
u=Q\{g(k * O) \Pi+n\} \cdot d,
$$

where $O$ represents the photonic flux (in a given wavelength band), $k$ is the point spread function of the optical-captor joint apparatus, $*$ denotes the convolution operator, $\Pi$ is a sampling operator, i.e. a Dirac comb supported by the centers of the matrix of digital sensors, $g$ is a nonlinear contrast change characterizing the nonlinear response of the sensors, $n$ represents a random perturbation due to photonic or electronic noise, $Q$ is a uniform quantization operator mapping $\mathbb{R}$ to a discrete interval of values, typically $[0,255]$, and $d$ represents an impulse noise due to transmission. Each one of the operations involved in (1) is at the basis of one of the main theories of signal processing. For instance, Shannon theory fixes the conditions under which we can recover $k * O$ from the sampled signal $(k * O) \Pi$, assuming that $k * O$ is a bandlimited function, i.e., its frequency range has compact support.

\section{Nonlinear contrast changes and level sets}

Let us focus on the consequences of the nonlinear contrast change $g$ for image processing. In human communication, none of the camera parameters is known to the observer; in most cases this information is lost when the image $u$ is used. This loss is rather the rule for the contrast change $g$. The informations about $g$ are inasmuch neglected as they are generally irrelevant: indeed, the contrast of an image widely depends on the sensor's properties but also on the lighting conditions 
and finally on the objects' temporary reflection properties: these conditions are anyway unknown! This led the physicist and gestaltist M. Wertheimer [68] to state as a principle that the grey level is not an observable. Images are observed up to an arbitrary and unknown contrast change.

An image analysis doctrine, the so called Mathematical Morphology, has recognized contrast invariance as a basic invariance requirement and proposed that image analysis operations should take into account this invariance principle [60]. With this principle, an image $u$ is a representative of an equivalence class of images $v$ obtained from $u$ via a contrast change, i.e., $v=g(u)$ where $g$, for simplicity, will be a continuous strictly increasing function. Under this assumption, an image is characterized by its upper (or lower) level sets $X_{\lambda}=\{x: u(x) \geq \lambda\}$ (resp. $\left.X_{\lambda}^{\prime}=\{x: u(x) \geq \lambda\}\right)$. Moreover, the image can be recovered from its level sets by the reconstruction formula

$$
u(x)=\sup \left\{\lambda: x \in X_{\lambda}\right\} .
$$

As it is easily seen, the family of the level sets (upper or lower) of $u$ is invariant under continuous strictly increasing contrast changes. An image operator $T$ is contrast invariant if

$$
T(g(u))=g(T(u))
$$

for any continuous strictly increasing contrast change $g$ and any image $u$. In particular, many efficient denoising operators respect this principle. See a classification of contrast invariant image multiscale smoothing operators in [2].

\section{Connected components of level sets}

Level sets are therefore basic objects for image processing and analysis. They have been acknowledged as such in several shape analysis theories, where thresholding is the basic image analysis operator [34]. Very early in image processing, authors noticed that to find a single and the right threshold in an image was enough to deliver a binary image with most of the relevant shape information. Theories of the "optimal threshold" were even developed [69]. In order to have a more local description of the basic objects of an image, several authors $([12,60])$ proposed to consider the connected components of (upper or lower) level sets as the basic objects of the image. They argue that contrast changes are local and depend upon the reflectance properties of objects. Thus, not only global contrast, but also local contrast is irrelevant. In [12], a notion of local contrast change is defined and it is proved that only connected components of level sets are invariant under such contrast changes. This approach was generalized in [6] where the authors compare different satellite images of the same landscape, taken at different times or in different channels. They show that these images have many connected components of bilevel sets in common (we call bilevel set any set $\{x, a \leq u(x) \leq b\}$ ). This same technique has been recently extended in [48] to image registration, one of the most basic tools in multiimage processing. Image registration based on connected components of level sets is shown to work efficiently where classical 
correlation techniques fail: when both registered images do not correspond to almost simultaneous snapshots. If $u$ belongs to a function space such that each connected component of a level set is bounded by a countable or finite number of oriented Jordan curves, we call topographic map the family of these Jordan curves [12]. In [44], a disocclusion method is developed, which restores images with spots or missing parts. This method computes Jordan curves in the image as boundaries of level sets and interpolates them in the missing parts.

\section{A nested Jordan curves representation}

Following [12], P. Monasse and F. Guichard [49] proposed, in a discrete framework, a fast and consistent discrete algorithm to compute a topographic map: they consider connected components of level sets, then they define a tree, ordered by inclusion, in the following way: they construct (in a discrete framework) a uniquely defined Jordan curve surrounding each connected component of each upper level set. In the same way, they consider all external Jordan curves of all connected components of lower level sets of the same image. Provided connectedness is adequately defined in the discrete grid (this definition is different for the upper level sets and the lower level sets!) they show that both systems of Jordan curves fuse into one, such that no pair of Jordan curves crosses. In this way, they obtain a topographic map, i.e. a system of Jordan lines organized by inclusion as a tree. They call this digital representation "fast level set transform" and it provides a fast numerical access to any connected region of the image and any "shape", understood as a Jordan curve surrounding a region. They let notice by some examples, however, that the inclusion trees of $u$ and $-u$ are not necessarily identical.

\section{WBV: Functions whose level sets have finite perimeter}

One of the main purposes of this paper is to justify the assumptions underlying the above mentioned methods. We shall define a functional model for $u$ where it is possible to define a notion of connected components for the level sets of $u$. Boundary of these connected components must consist of a countable or finite number of oriented Jordan curves from which we can recover the set by the obvious filling algorithm. This functional model, called WBV, is a variant of the space of functions of bounded variation. Indeed, WBV functions are BV functions modulo a change of contrast, i.e. for any $u \in \mathrm{WBV}$ there exists a bounded, continuous and strictly increasing contrast change $g$ such that $g(u)$ is a function of bounded variation. The space of functions of bounded variation is a sound model for images which have discontinuities and it has been frequently used as a functional model for the purposes of image denoising, edge detection, etc. [56]. L. Rudin [55] proposed that images should be handled as functions with bounded variation. He used the classical result of geometric measure theory [29] that the essential discontinuity set of a BV function is rectifiable and argued that the "edge set" sought in edge detection theory $[42,43]$, was nothing but this discontinuity set. An indirect confirmation of this thesis is given by the variational image segmentation 
theory. Indeed, a paradigmatic variational model proposed by Mumford-Shah [52] finds naturally its minima in a class of functions with bounded variation, SBV [3, $4,17,18]$. A full account can be found in the book [5].

As a consequence of the results discussed in this paper, we shall show that all of the mentioned approaches, Mathematical Morphology, BV model, shapes described by Jordan curves or by connected regions, fast level set transform are compatible with a single underlying functional model, WBV. We shall introduce the " $M$-connectedness" as the right notion of connectedness for sets of finite perimeter. We shall develop this formalism in full generality for sets of finite perimeter in $\mathbb{R}^{\mathrm{N}}$. For sets of finite perimeter in $\mathbb{R}^{2}$ a more precise description is possible, since in this case, the essential boundary of each $M$-connected component can be described as a countable or finite union of rectifiable Jordan curves. Since almost all level sets of functions in WBV are sets of finite perimeter, then level sets of WBV functions can be described in terms of rectifiable Jordan curves and we get a description of the shapes in an image which is both complete and well-founded.

\section{Image denoising or segmentation operators based on connected components}

The use of connected components of level sets has become recently very relevant in a series of image filters introduced in Mathematical Morphology. Motivated by the study of a family of filters by reconstruction [37,38,57,64,65], J. Serra and $\mathrm{Ph}$. Salembier $[58,62]$ introduced the notion of connected operators. To be precise, Serra and Salembier call connected an operator $\psi$ on sets if, for each family of sets $A$, the partition of the image domain associated to $\psi(A)$ (i.e., the partition of the image domain made of the connected components of $\psi(A)$ and the connected components of its complement) is less fine than the partition associated to $A$ (i.e., the partition of the image domain made of the connected components of $A$ and the connected components of its complement). Such operators simplify the topographic map of the image. These filters have become very popular because, on an experimental basis, they have been claimed to simplify the image while preserving contours. This property has made them very attractive for a large number of applications such as noise cancellation [64,65] or segmentation [47,66]. More recently, they have become the basis of a morphological approach to image and video compression (see [59] and references therein, and more recently [27]).

\section{Application to connected operators}

As an application of the theory of $M$-connected components for sets of finite perimeter developed here, we study the L. Vincent filters (filters which, when defined on sets, remove the connected components of small measure). We show that these filters can be defined on functions of bounded variation and, more generally, in WBV. We prove that they define contrast invariant filtering operators which are well behaved also in the classical Sobolev and BV spaces and simplify the connected components of the upper and/or lower level sets of the image (see also [44]). 


\section{An objection to the $\mathrm{BV}$ model}

Before closing with this introduction, it may be useful to answer to an obvious objection: according to the classical model given by (1), the raw image $O$ may be $\mathrm{BV}$, but the digital image $g(k * O)$ is more regular, at least, say, $\mathrm{C}^{1}$ if $g$ is and if the image formation follows Shannon conditions. Thus, we might as well have worked in a space of continuous functions. In this framework, connected components can be defined in the classical way and Jordan curves obtained in the image by Sard Lemma and the Implicit Functions theorem. To take this assumption would save all of the effort spent here. The answer to this objection comes from technology. There is no evidence in all of the works dedicated to image processing in favour of any advantage taken of a regularity assumption for the images. Because of the three noises present in image caption (transmission impulse noise, gaussian quantum noise of sensors, quantization noise), the image cannot be considered as a continuous function. In many cases, Shannon conditions are imperfectly satisfied. In addition, the BV model makes sense for the subjacent "real" image $O$, which presents rectifiable discontinuity lines along all apparent contours of objects. Thus, $O$ is at least as discontinuous as a BV function, and probably more. In fact, an experimental procedure can be defined [1] to check whether the subjacent image is in BV or not: the results seem to indicate that most images are too oscillating to belong to $\mathrm{BV}$. We mentioned that both restoration and segmentation models try with success to project back in some more or less nonlinear way the image onto BV [56]. This is also true for the recent "wavelet shrinkage" method for image denoising [19] or image deconvolution [20]. Last but not least, the discrete representations used in Mathematical Morphology [60] are not more regular than BV and the recent image compression standards aim at the delivery of a BV compressed image. To summarize, the BV model is probably too smooth for the "real" subjacent image (i.e. the photonic flux), but seems to be on the way to be acknowledged as the right model to describe the digital images handled in technology. We may add the results of the present work as one more argument in favour of the BV model (and the variant $\mathrm{WBV}$ we propose) as a common denominator to image analysis and restoration.

\section{Plan of this paper}

This paper is organized as follows. Sections 2 and 3 introduce some basic facts about Caccioppoli sets and BV functions. In Sect. 4 we study in detail a definition of $M$-connectedness for sets with finite perimeter, first proposed by H. Federer in the more general framework of the theory of currents. We compare this concept with the conventional topological one and give a new proof, based on a simple variational argument, of the existence and uniqueness of the decomposition into $M$-connected components. Section 5 explains how to "fill the holes", or to "saturate", an indecomposable set. Section 6 defines Jordan boundaries (which correspond in dimension 2 to Jordan curves) and gives a unique decomposition theorem (Theorem 4) of the essential boundary into Jordan boundaries, with their structure. Theorem 5 gives a converse statement and a reconstruction formula of 
a Caccioppoli set from its set of Jordan boundaries. In Sect. 7 we construct for any Caccioppoli set $E$ a "topographic function", an integer valued BV function whose boundaries of upper level sets yield all Jordan boundaries of $E$. In this way, the Jordan boundaries of $E$ benefit of the obvious inclusion structure of the upper level sets of $u$ and are numbered in odd and even levels of $u$, following their level of inclusion and their classification into set, versus hole, boundaries. In Sect. 8, we give the two dimensional interpretation of these results and show that in this case the link with conventional topology is much stronger: indeed, we show that the essential boundary of any simple set $E$ (i.e. such that both $E$ and $\mathbb{R}^{2} \backslash E$ are indecomposable) is equivalent, modulo $\mathcal{H}^{1}$-negligible sets, to a Jordan curve (this result was first proved by W.H. Fleming in [25]) and also that for any indecomposable set $E$ there exists a canonical set $F$ equivalent to $E$ which is connected by rectifiable arcs. Section 9 is devoted, as an illustration, to a case study in image denoising. We show the good definition and properties of the above mentioned Vincent-Serra "connected operators" in WBV and in the classical Sobolev and BV spaces. In particular, we prove that these operators, notwithstanding their nonlocal nature, map $\mathrm{W}^{1, p}$ in $\mathrm{W}^{1, p}$ for any $p \in[1, \infty]$ and do not increase a.e. the modulus of the gradient. In this respect, quite surprisingly, they behave as the usual local truncation operators.

\section{Notation and main facts about sets of finite perimeter}

We consider a $N$-dimensional euclidean space $\mathbb{R}^{\mathrm{N}}$, with $N \geq 2$. The Lebesgue measure of a Lebesgue measurable set $E \subseteq \mathbb{R}^{\mathrm{N}}$ will be denoted by $|E|$. For a Lebesgue measurable subset $E \subseteq \mathbb{R}^{\mathrm{N}}$ and a point $x \in \mathbb{R}^{\mathrm{N}}$, the upper and lower densities of $E$ at $x$ are respectively defined by

$$
\bar{D}(E, x):=\limsup _{r \rightarrow 0^{+}} \frac{|E \cap B(x, r)|}{|B(x, r)|}, \quad \underline{D}(E, x):=\liminf _{r \rightarrow 0^{+}} \frac{|E \cap B(x, r)|}{|B(x, r)|} .
$$

If the upper and lower densities are equal, their common value will be called the density of $E$ at $x$ and it will be denoted by $D(E, x)$. We shall use the word measurable to mean Lebesgue measurable.

Using densities we can define the essential interior $\stackrel{\circ}{E}^{\mathrm{M}}$, the essential closure $\bar{E}^{\mathrm{M}}$ and the essential boundary $\partial^{\mathrm{M}} E$ of a measurable set $E$ as follows:

$$
\begin{aligned}
\stackrel{\circ}{E}^{\mathrm{M}} & :=\{x: D(E, x)=1\}, \quad \bar{E}^{\mathrm{M}}:=\{x: \bar{D}(E, x)>0\} \\
\partial^{\mathrm{M}} E & :=\bar{E}^{\mathrm{M}} \cap{\overline{\mathbb{R}^{\mathrm{N}} \backslash E}}^{\mathrm{M}}=\left\{x: \bar{D}(E, x)>0, \bar{D}\left(\mathbb{R}^{\mathrm{N}} \backslash E, x\right)>0\right\} .
\end{aligned}
$$

Notice also that by the Lebesgue differentiation theorem the symmetric difference $\stackrel{\circ}{E}^{\mathrm{M}} \Delta E$ is Lebesgue negligible, hence the measure theoretic interior of $\stackrel{\circ}{E}^{\mathrm{M}}$ is $\stackrel{\circ}{E}^{\mathrm{M}}$ (in this sense $\stackrel{\circ}{\mathrm{M}}^{\mathrm{M}}$ is essentially open), and also that

$$
\partial^{\mathrm{M}} E=\mathbb{R}^{\mathrm{N}} \backslash\left(\stackrel{\circ}{E}^{\mathrm{M}} \cup{{\mathbb{\mathbb { R } ^ { \mathrm { N } } \backslash E}}^{\mathrm{M}}}^{\circ}\right. \text {. }
$$

We also use the notation $E^{1 / 2}$ to indicate the set of points where the density of $E$ is $1 / 2$. 
Here and in what follows we shall denote by $\mathcal{H}^{\alpha}$ the Hausdorff measure of dimension $\alpha$ in $\mathbb{R}^{\mathrm{N}}$. In particular, $\mathcal{H}^{N-1}$ denotes the $(N-1)$-dimensional Hausdorff measure and $\mathcal{H}^{N}$, the $N$-dimensional Hausdorff measure, coincides with the (outer) Lebesgue measure in $\mathbb{R}^{\mathrm{N}}$. Given any Borel set $B \subseteq \mathbb{R}^{\mathrm{N}}$ with $\mathcal{H}^{\alpha}(B)<\infty$, we denote by $\mathcal{H}^{\alpha}\left\llcorner B\right.$ the finite Borel measure $\chi_{B} \mathcal{H}^{\alpha}$, i.e. $\mathcal{H}^{\alpha}\left\llcorner B(C)=\mathcal{H}^{\alpha}(B \cap C)\right.$ for any Borel set $C \subseteq \mathbb{R}^{\mathrm{N}}$. We recall that

$$
\lim _{r \rightarrow 0^{+}} \frac{\mathcal{H}^{k}(B \cap B(x, r))}{r^{k}}=0 \quad \text { for } \mathcal{H}^{k} \text {-a.e. } x \in \mathbb{R}^{\mathrm{N}} \backslash B
$$

holds whenever $B \subseteq \mathbb{R}^{\mathrm{N}}$ is a Borel set with finite $k$-dimensional Hausdorff measure (see for instance $\$ 2.3$ of [22]).

Given $A, B \subseteq \mathbb{R}^{\mathrm{N}}$, we shall write $E_{1}=E_{2}\left(\bmod \mathcal{H}^{\alpha}\right)$ if $H^{\alpha}\left(E_{1} \Delta E_{2}\right)=0$, where $E_{1} \Delta E_{2}=\left(E_{1} \backslash E_{2}\right) \cup\left(E_{2} \backslash E_{1}\right)$ is the symmetric difference of $E_{1}$ and $E_{2}$. We will use an analogous notation for the inclusion and in some cases, in order to simplify the notation, the equivalence or inclusion $\left(\bmod \mathcal{H}^{N}\right)$ will be tacitly understood.

We say that a measurable set $E \subseteq \mathbb{R}^{\mathrm{N}}$ has finite perimeter in $\mathbb{R}^{\mathrm{N}}$ if there exist a positive finite measure $\mu$ in $\mathbb{R}^{\mathrm{N}}$ and a Borel function $v_{E}: \mathbb{R}^{\mathrm{N}} \rightarrow \mathbf{S}^{N-1}$ (called generalized inner normal to $E$ ) such that the following generalized Gauss-Green formula holds

$$
\int_{E} \operatorname{div} \phi d x=-\int_{\mathbb{R}^{\mathrm{N}}}\left\langle v_{E}, \phi\right\rangle d \mu \quad \forall \phi \in C_{c}^{1}\left(\mathbb{R}^{\mathrm{N}}, \mathbb{R}^{\mathrm{N}}\right) .
$$

Hence the measure $v_{E} \mu$ is the distributional derivative of $\chi_{E}$, which will be denoted by $D \chi_{E}$, while $\mu=\left|D \chi_{E}\right|$ is its total variation; the perimeter $P(E, B)$ of $E$ in a Borel set $B \subseteq \mathbb{R}^{\mathrm{N}}$ is defined by $\left|D \chi_{E}\right|(B)$, and we use the notation $P(E)$ in the case $B=\mathbb{R}^{\mathrm{N}}$.

The main facts concerning sets of finite perimeter that we will use in the following are listed below, for the reader's convenience (see for instance [5, 22, 24, $29,72])$.

- Criteria for the finiteness of perimeter

By Riesz theorem, a measurable set $E \subseteq \mathbb{R}^{\mathrm{N}}$ has finite perimeter if and only if

$$
\sup \left\{\int_{E} \operatorname{div} \phi d x: \phi \in C_{c}^{1}\left(\mathbb{R}^{\mathrm{N}}, \mathbb{R}^{\mathrm{N}}\right),|\phi| \leq 1\right\}<\infty
$$

and in this case the supremum equals the perimeter. A much deeper criterion is due to Federer: $E$ has finite perimeter in $\mathbb{R}^{\mathrm{N}}$ if and only if $\mathcal{H}^{N-1}\left(\partial^{\mathrm{M}} E\right)<\infty$ (if $\mathcal{H}^{N-1}(\partial E)<\infty$ the proof is much simpler, see for instance Proposition 3.62 of [5]). 
- Structure of $D \chi_{E}$

According to the De Giorgi and Federer theorems, for any set with finite perimeter the sets $E^{1 / 2}$ and $\partial^{\mathrm{M}} E$ have the same $\mathcal{H}^{N-1}$ measure, so that $\mathcal{H}^{N-1}\left(\partial^{\mathrm{M}} E \backslash E^{1 / 2}\right)=0$ and

$$
\mathcal{H}^{N-1}\left(\mathbb{R}^{\mathrm{N}} \backslash\left(\stackrel{\circ}{E}^{\mathrm{M}} \cup E^{1 / 2} \cup{\stackrel{\circ}{\mathbb{R}^{\mathrm{N}} \backslash E}}^{\mathrm{M}}\right)\right)=0 .
$$

So, at $\mathcal{H}^{N-1}$-a.e. point of $\mathbb{R}^{N}$ the density exists and belongs to $\{0,1 / 2,1\}$. Moreover

$$
\left|D \chi_{E}\right|=\mathcal{H}^{N-1}\left\llcorner\partial^{\mathrm{M}} E=\mathcal{H}^{N-1}\left\llcorner E^{1 / 2} .\right.\right.
$$

- Lower semicontinuity, approximation and compactness

The functional $E \mapsto P(E)$ (defined by (5), so that $P(E)=\infty$ if $E$ has not finite perimeter) is lower semicontinuous with respect to local convergence in measure in $\mathbb{R}^{\mathrm{N}}$ (i.e. $L_{\text {loc }}^{1}$ convergence of the characteristic functions); moreover, for any set $E$ with $P(E)<\infty$ there exists a sequence of sets $E_{h}$ with smooth boundary locally converging in measure to $E$ and such that $P(E)=\lim _{h} P\left(E_{h}\right)$. Any sequence of sets with equibounded perimeters admits subsequences locally converging in measure.

- Isoperimetric inequalities

If $E \subseteq \mathbb{R}^{\mathrm{N}}$ has finite perimeter, then either $E$ or $\mathbb{R}^{\mathrm{N}} \backslash E$ have finite measure and the isoperimetric inequality holds:

$$
\min \left\{|E|^{\frac{N-1}{N}},\left|\mathbb{R}^{\mathrm{N}} \backslash E\right|^{\frac{N-1}{N}}\right\} \leq \gamma_{N} P(E) .
$$

Denoting by $\omega_{N}$ the measure of the unit ball $B(0,1)$, the optimal isoperimetric constant is $\omega_{N}^{-1 / N} / N$ (see [16]). A local counterpart of this inequality is the relative isoperimetric inequality:

$$
\min \{|B(x, r) \cap E|,|B(x, r) \backslash E|\} \leq \eta_{N} r \mathcal{H}^{N-1}\left(\partial^{\mathrm{M}} E \cap B(x, r)\right) .
$$

\section{BV functions and related spaces}

In this section we recall some definitions and properties related to the space of functions with bounded variation in $\Omega$, denoted by $\mathrm{BV}(\Omega)$.

Given a Borel function $u: \Omega \rightarrow[-\infty,+\infty]$, the approximate lower and upper limits $u^{-}, u^{+}: \Omega \rightarrow[-\infty,+\infty]$ are Borel functions defined at every point $x \in \Omega$ as follows: $u^{-}(x)$ is the supremum of all those $t \in[-\infty,+\infty]$ such that $x \in \overbrace{\{u \geq t\}}^{\mathrm{M}}$ whereas $u^{+}(x)$ is the infimum of all those $t \in[-\infty,+\infty]$ such that $x \in{\overbrace{\{u t t}}^{\mathrm{M}}$. The set

$$
S_{u}:=\left\{x \in \Omega: u^{-}(x)<u^{+}(x)\right\}
$$


is called the approximate discontinuity set of $u$ and is negligible with respect to the Lebesgue measure. The function $u$ is said to be approximatively continuous at any point $x \in \Omega \backslash S_{u}$ and we shall denote

$$
\text { ap } \lim _{y \rightarrow x} u(y)=u^{-}(x)=u^{+}(x) \quad \forall x \in \Omega \backslash S_{u}
$$

Let $x \in \Omega \backslash S_{u}$ such that $\operatorname{ap} \lim u(x) \in \mathbb{R}$. We say that $u$ is approximatively differentiable at $x$ if there exists a vector $\nabla u(x)$ such that the sets

$$
\left\{y \in \Omega \backslash\{x\}: \frac{|u(y)-\operatorname{ap} \lim u(x)-\langle\nabla u(x), y-x\rangle|}{|y-x|}>\epsilon\right\}
$$

have 0 density in $x$ for every $\epsilon>0$.

We define $\mathrm{BV}(\Omega)$ as the space of all those functions $u \in \mathrm{L}^{1}(\Omega)$ whose distributional derivative is representable as a $\mathbb{R}^{\mathrm{N}}$-valued measure $D u=\left(D_{1} u, \ldots, D_{N} u\right)$ with finite total variation in $\Omega$, i.e.

$$
\int_{\Omega} u \operatorname{div} \phi d x=-\sum_{i=1}^{N} \int_{\Omega} \phi_{i} d D_{i} u \quad \forall \phi \in\left[\mathrm{C}_{c}^{1}(\Omega)\right]^{N} .
$$

The total variation $|D u|$ of a BV function $u$ is defined as the total variation of the vector measure $D u$. The space $\mathrm{BV}(\Omega)$ is endowed with the norm $\|u\|_{\mathrm{BV}}=$ $\|u\|_{\mathrm{L}^{1}}+|D u|(\Omega)$. We shall denote by $\mathrm{BV}_{\text {loc }}(\Omega)$ the space of all those functions that belong to $\operatorname{BV}(\tilde{\Omega})$ for every open set $\tilde{\Omega} \subset \subset \Omega$. In view of Sect. 2 , it is easily seen that a subset $E \subset \mathbb{R}^{\mathrm{N}}$ has finite perimeter in $\Omega$ if and only if $u=\chi_{E} \in \mathrm{BV}_{\text {loc }}(\Omega)$ and $|D u|(\Omega)<\infty$. Main properties of BV functions are the following (see for instance $[5,22,24,29,72])$ :

- Lower semicontinuity of the variation measure Suppose $\left\{u_{n}\right\}_{n \in \mathbb{N}} \subset \mathrm{BV}(\Omega)$ and $u_{n} \rightarrow u$ in $\mathrm{L}_{\text {loc }}^{1}(\Omega)$ then

$$
|D u|(\Omega) \leq \liminf _{n \rightarrow \infty}\left|D u_{n}\right|(\Omega)
$$

- Approximation by smooth functions

Assume $u \in \mathrm{BV}(\Omega)$. There exist functions $u_{n} \in \mathrm{BV}(\Omega) \cap \mathrm{C}^{\infty}(\Omega)$ such that

$$
u_{n} \rightarrow u \text { in } \mathrm{L}^{1}(\Omega) \quad \text { and } \quad\left|D u_{n}\right|(\Omega) \rightarrow|D u|(\Omega) \text { as } n \rightarrow \infty
$$

- Compactness

If $\left\{u_{n}\right\}$ is a sequence in $\mathrm{BV}(\Omega)$ satisfying $\sup _{n}\left\|u_{n}\right\|_{\mathrm{BV}}<\infty$, then there exist a subsequence $\left\{u_{n_{k}}\right\}$ and a function $u \in \operatorname{BV}(\Omega)$ such that

$$
u_{n_{k}} \rightarrow u \text { in } \mathrm{L}_{\text {loc }}^{1}(\Omega)
$$




\section{- Poincaré inequality}

If $\Omega$ is bounded, connected and with Lipschitz boundary, then there exists a constant $C$ such that

$\int_{\Omega \cap B(x, r)}|u-\bar{u}| \leq C|D u|(B(x, r) \cap \Omega)$ for all balls $B(x, r) \subset \mathbb{R}^{\mathrm{N}}$ and $u \in \mathrm{BV}(\Omega)$

where $\bar{u}(x)=f_{\Omega \cap B(x, r)} u(y) d y$.

- Coarea formula

Let $u \in \operatorname{BV}(\Omega)$. Then $\{u>t\}$ has finite perimeter in $\Omega$ for $\mathrm{L}^{1}$-a.e. $t \in \mathbb{R}$ and

$$
|D u|(\Omega)=\int_{-\infty}^{+\infty} P(\{u>t\}, \Omega) d t
$$

Conversely, if $u \in \mathrm{L}^{1}(\Omega)$ and $\int_{-\infty}^{+\infty} P(\{u>t\}, \Omega) d t<\infty$ then $u \in \operatorname{BV}(\Omega)$. In addition, notice that $P(\{u>t\}, \Omega)=P(\{u<t\}, \Omega)$ since the fact that $u$ is measurable is enough to ensure that $|\{u=t\}|>0$ for at most countably many $t \in \mathbb{R}$.

- Rectifiability of $S_{u}$ and approximate jump set $J_{u}$

Let $u \in \operatorname{BV}(\Omega)$. Then $S_{u}$ is countably $(N-1)$-rectifiable and $-\infty<u^{-}(x) \leq$ $u^{+}(x)<+\infty$ for $\mathcal{H}^{\mathrm{N}-1}$-almost every $x \in \Omega$. In addition, for $\mathcal{H}^{N-1}$-a.e. $x \in S_{u}$ there exists a unique unit vector $v_{u} \in S^{N-1}$ such that, setting $B_{r}^{+}\left(x, v_{u}\right):=\{y \in$ $\left.B_{r}(x):\left\langle y-x, v_{u}\right\rangle>0\right\}$ and $B_{r}^{-}(x, v):=\left\{y \in B_{r}(x):\left\langle y-x, v_{u}\right\rangle<0\right\}$,

$$
\lim _{r \downarrow 0}\left[f_{B_{r}^{+}\left(x, v_{u}\right)}\left|u(y)-u^{+}(x)\right| d y+f_{B_{r}^{-}(x, v)}\left|u(y)-u^{-}(x)\right| d y\right]=0 .
$$

The set of points where this equality occurs is called the approximate jump set and denoted as $J_{u}$. Hence, $\mathcal{H}^{N-1}\left(S_{u} \backslash J_{u}\right)=0$ and $D u$ vanishes on $S_{u} \backslash J_{u}$.

- Decomposition of the derivative

Let $u \in \mathrm{BV}(\Omega)$. Then $D u$ can be decomposed into three parts:

$$
D u=D^{a} u+D^{j} u+D^{c} u
$$

where $D^{a} u$ is the absolutely continuous part of $D u$ with respect to $\mathcal{L}^{N}$ and, denoting by $D^{s} u$ the singular part of $D u$ with respect to $\mathcal{L}^{N}, D^{j} u:=D^{s}\left\llcorner J_{u}\right.$ and $D^{c} u:=$ $D^{s} u\left\llcorner\left(\Omega \backslash S_{u}\right) . D^{j} u\right.$ is called the jump part of the derivative and $D^{c} u$ the Cantor part of the derivative. Then $D^{a} u=\nabla u \mathcal{L}^{n}, D^{j} u=D u\left\llcorner J_{u}=\left(u^{+}-u^{-}\right) v_{u} \mathcal{H}^{N-1}\left\llcorner J_{u}\right.\right.$ and $D^{c} u$ vanishes on sets which are $\sigma$-finite with respect to $\mathcal{H}^{N-1}$.

Several functional spaces were introduced in [3] (see also [54]) to offer a reliable framework for some minimization problems issuing from image processing and the mathematical theory of liquid crystals. We shall concentrate on the space of generalized functions of bounded variation $\operatorname{GBV}(\Omega)$, which can be defined as 
follows: we say that $u: \Omega \rightarrow[-\infty,+\infty]$ is a generalized function of bounded variation if

$$
u^{N}:=(-N) \vee u \wedge N \in \mathrm{BV}(\Omega) \quad \forall N \in \mathbb{N},
$$

which means that all truncations of $u$ have bounded variation. For the sake of simplicity, we have chosen to work with $\mathrm{BV}(\Omega)$ rather than $\mathrm{BV}_{\text {loc }}(\Omega)$, which is the definition adopted in [3]. Remark that GBV functions are not summable in general. Let us now define the function $m_{u}: \mathbb{R} \rightarrow[0, \infty]$ as

$$
m_{u}(t):=P(\{u>t\}, \Omega) .
$$

Lemma 1. Let $u: \Omega \rightarrow[-\infty,+\infty]$ be a Borel function such that $u \not \equiv+\infty$ and $u \not \equiv-\infty$ up to Lebesgue negligible sets. Then the following propositions hold:

(i) if $\Omega$ is bounded then $m_{u} \in \mathrm{L}_{\mathrm{loc}}^{1}(\mathbb{R})$ if and only if $u \in \operatorname{GBV}(\Omega)$.

(ii) if $\Omega$ is bounded, connected and with Lipschitz boundary then $m_{u} \in \mathrm{L}^{1}(\mathbb{R})$ if and only if $u \in \mathrm{BV}(\Omega)$.

Proof. (i) $(\Leftarrow)$ By definition, $u^{N} \in \mathrm{BV}(\Omega)$ for every $N \in \mathbb{N}$. Since, for any $N \in \mathbb{N},\{u>t\}=\left\{u^{N}>t\right\}$ for every $t \in(-N, N)$ we get by the coarea formula applied to the truncated function

$$
\int_{-N}^{N} P(\{u>t\}, \Omega) d t=\int_{-N}^{N} P\left(\left\{u^{N}>t\right\}, \Omega\right) d t \leq\left|D u^{N}\right|(\Omega)<+\infty
$$

for every $N \in \mathbb{N}$.

Therefore, $m_{u} \in \mathrm{L}_{\text {loc }}^{1}(\mathbb{R})$.

$(\Rightarrow)$ First recall the well-known equality for Borel functions

$$
u(x)=\int_{0}^{+\infty} \chi_{\{u>t\}}(x) d t-\int_{-\infty}^{0}\left(1-\chi_{\{u>t\}}\right)(x) d t \quad \forall x \in \Omega .
$$

Given $\phi \in \mathrm{C}_{\mathrm{c}}^{1}\left(\Omega ; \mathbb{R}^{\mathrm{N}}\right)$ with $\|\phi\|_{\infty} \leq 1$, we use Riesz Theorem applied to the upper level sets, Fubini's Theorem and the fact that the integral of $\operatorname{div} \phi$ is zero to get for every $N \in \mathbb{N}$

$$
\begin{aligned}
\int_{\Omega} u^{N} \operatorname{div} \phi d x & =\int_{\Omega} \int_{-N}^{+N} \chi_{\left\{u^{N}>t\right\}} \operatorname{div} \phi d x d t \\
& =\int_{-N}^{+N} \int_{\Omega} \chi_{\{u>t\}} \operatorname{div} \phi d x d t \\
& \leq \int_{-N}^{+N} P\left(\chi_{\{u>t\}}, \Omega\right) d t<+\infty
\end{aligned}
$$

By Riesz Theorem, this implies that $u^{N} \in \mathrm{BV}(\Omega)$ for every $N \in \mathbb{N}$.

(ii) $(\Leftarrow)$ is a straightforward consequence of the coarea formula.

$(\Rightarrow)$ It follows from (i) that $u \in \operatorname{GBV}(\Omega)$. Using Poincaré inequality we get that for every $N \in \mathbb{N}$

$$
\int_{\Omega}\left|u^{N}-\overline{u^{N}}\right| d x \leq C\left|D u^{N}\right|(\Omega) \leq C \int_{-\infty}^{+\infty} P(\{u>t\}, \Omega) d t=C_{1}
$$


with $\overline{u^{N}}=f_{\Omega} u^{N}(y) d y$. Now, let us prove that the sequence $\overline{\left|u^{N}\right|}$ is bounded. Assume that for some sequence $N_{i} \in \mathbb{N}, \overline{u^{N_{i}}} \rightarrow+\infty$ (the argument is analogous if $\left.\overline{u^{N_{i}}} \rightarrow-\infty\right)$ and let $\Omega_{M}=\{u \in[-\infty, M]\}$. Then, for $i$ large enough

$$
\left.\overline{\left(u^{N_{i}}\right.}-M\right)\left|\Omega_{M}\right| \leq \int_{\Omega}\left|u^{N}-\overline{u^{N}}\right| d x \leq C_{1}
$$

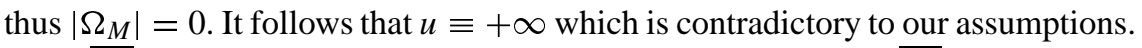
Thus $\left|\overline{u^{N}}\right|$ is bounded and, possibly by extracting a subsequence, $\overline{u^{N}} \rightarrow z$. Finally, letting $N \rightarrow \infty$, we get that

$$
\int_{\Omega}|u-z| d x \leq C_{1}
$$

which implies that $u \in \mathrm{L}^{1}(\Omega)$ and $u$ is real-valued. Then $u \in \mathrm{BV}(\Omega)$ by a simple application of the coarea formula.

\section{Decomposability of a set with finite perimeter}

Let $E \subseteq \mathbb{R}^{\mathrm{N}}$ be a set with finite perimeter. We say that $E$ is decomposable if there exists a partition $(A, B)$ of $E$ such that $P(E)=P(A)+P(B)$ and both $|A|$ and $|B|$ are strictly positive. We say that $E$ is indecomposable if it is not decomposable; notice that the properties of being decomposable or indecomposable are invariant $\left(\bmod \mathcal{H}^{N}\right)$ and that, according to our definition, any Lebesgue negligible set is indecomposable.

It is natural to compare this definition with the topological one of connectedness: no implication is trivial in general, since on one hand in the definition of indecomposability the sets $A, B$ are not required to be relatively open, but on the other hand they are required to be sets of finite perimeter. We will see that in some cases a comparison is possible, especially in the case of subsets of the plane, and that in any case all formal properties satisfied by connected sets are fulfilled in this slightly different setting.

We start our investigation by analyzing the situations in which the equality $P(A \cup B)=P(A)+P(B)$ occurs.

Proposition 1. Let A, B be sets of finite perimeter. Then

$$
P(A \cup B)+P(A \cap B) \leq P(A)+P(B)
$$

and

$$
P(A)+P(B)=P(A \cup B)+2 \mathcal{H}^{N-1}\left(\partial^{\mathrm{M}} A \cap \partial^{\mathrm{M}} B\right) \quad \text { whenever }|A \cap B|=0 .
$$

Proof. The following inclusions are a straightforward consequence of the definition of $\partial^{\mathrm{M}}$ :

$\partial^{\mathrm{M}}(A \cup B) \cup \partial^{\mathrm{M}}(A \cap B) \subset \partial^{\mathrm{M}} A \cup \partial^{\mathrm{M}} B, \quad \partial^{\mathrm{M}}(A \cup B) \cap \partial^{\mathrm{M}}(A \cap B) \subset \partial^{\mathrm{M}} A \cap \partial^{\mathrm{M}} B$. 
Taking into account that $P(E)=\mathcal{H}^{N-1}\left(\partial^{\mathrm{M}} E\right)$ for any set of finite perimeter $E$, the first inequality follows. If $|A \cap B|=0$ we denote by $L$ the $\mathcal{H}^{N-1}$-negligible set $\left(\partial^{\mathrm{M}} A \backslash A^{1 / 2}\right) \cup\left(\partial^{\mathrm{M}} B \backslash B^{1 / 2}\right)$ and notice that

$$
\partial^{\mathrm{M}}(A \cup B) \backslash L \subset \partial^{\mathrm{M}} A \Delta \partial^{\mathrm{M}} B, \quad \partial^{\mathrm{M}} A \Delta \partial^{\mathrm{M}} B \subset \partial^{\mathrm{M}}(A \cup B)
$$

hence $P(A \cup B)=\mathcal{H}^{N-1}\left(\partial^{\mathrm{M}} A \Delta \partial^{\mathrm{M}} B\right)$. From this fact the second identity easily follows.

As an application of Proposition 1 we can prove that any open connected set with finite perimeter is indecomposable. We will obtain a converse property in Theorem 2 (see also Theorem 8, for domains in the plane).

Proposition 2 (Connectedness and indecomposability). Any connected open set $\Omega \subseteq \mathbb{R}^{\mathrm{N}}$ satisfying $\mathcal{H}^{N-1}\left(\partial^{\mathrm{M}} \Omega\right)<\infty$ is indecomposable.

Proof. By Federer's theorem, we know that $\Omega$ has finite perimeter. Let $(A, B)$ be a partition of $\Omega$ such that $P(\Omega)=P(A)+P(B)$. Then, since

$$
\partial^{\mathrm{M}} A \subset \partial^{\mathrm{M}} B \cup \partial^{\mathrm{M}} \Omega
$$

and, by Proposition $1, \partial^{M} A \cap \partial^{M} B=\emptyset\left(\bmod \mathcal{H}^{N-1}\right)$, we have

$$
\mathcal{H}^{N-1}\left(\Omega \cap \partial^{\mathrm{M}} A\right) \leq \mathcal{H}^{N-1}\left(\Omega \cap \partial^{\mathrm{M}} \Omega\right)=0
$$

hence $D \chi_{A}=0$ in $\Omega$. This proves that $\chi_{A}$ is locally equivalent to a constant in $\Omega$, and, being $\Omega$ connected, this is true globally.

Another simple consequence of Proposition 1 is the subadditivity of perimeter

$$
P\left(\bigcup_{i \in I} A_{i}\right) \leq \sum_{i \in I} P\left(A_{i}\right)
$$

for finite or countable families. For finite families the proof is achieved by induction and for countable ones one can use the lower semicontinuity of the perimeter with respect to the local convergence in measure.

Now we extend our analysis to finite or countable families of sets; this extension is necessary in view of the treatment of the family of indecomposable components of a set. A more comprehensive treatment of the properties of partitions in finitely or countably many sets of finite perimeter (the so-called Caccioppoli partitions) is given in the paper [13] by G. Congedo and I. Tamanini (see also Chapter 4 of [5] and [39]); here we only prove the properties that will be needed in the following.

Proposition 3. Let I be a finite or countable set, let $\left\{A_{i}\right\}_{i \in I}$ be a family of sets of finite perimeter and let $A$ be their union. Then, assuming that $A_{i} \neq \mathbb{R}^{\mathrm{N}}$ for any $i \in I$ and $\sum_{i} P\left(A_{i}\right)<\infty$, the following conditions are equivalent:

(i) $P(A) \geq \sum_{i} P\left(A_{i}\right)$;

(ii) $P(A)=\sum_{i} P\left(A_{i}\right)$;

(iii) for any $i \neq j$ we have $\left|A_{i} \cap A_{j}\right|=0$ and $\mathcal{H}^{N-1}\left(\partial^{\mathrm{M}} A_{i} \cap \partial^{\mathrm{M}} A_{j}\right)=0$;

(iv) for any $i \neq j$ we have $\left|A_{i} \cap A_{j}\right|=0$ and $\cup_{i} \partial^{\mathrm{M}} A_{i} \subset \partial^{\mathrm{M}} A\left(\bmod \mathcal{H}^{N-1}\right)$. 
If these conditions are fulfilled we have also $\partial^{\mathrm{M}} A=\cup_{i} \partial^{\mathrm{M}} A_{i}\left(\bmod \mathcal{H}^{N-1}\right)$ and

$$
\mathcal{H}^{N-1}\left(\AA^{\mathrm{M}} \backslash \bigcup_{i \in I} \AA_{i}^{\mathrm{M}}\right)=0 .
$$

Proof. The equivalence between (i) and (ii) follows by the subadditivity of perimeter.

(ii) $\Longrightarrow$ (iii) For any pair of indexes $i, j \in I, i \neq j$, we have

$$
\begin{aligned}
P(A) & \leq P\left(A_{i} \cup A_{j}\right)+P\left(\bigcup_{k \in I \backslash\{i, j\}} A_{k}\right) \leq P\left(A_{i}\right)+P\left(A_{j}\right)+P\left(\bigcup_{k \in I \backslash\{i, j\}} A_{k}\right) \\
& \leq \sum_{k \in I} P\left(A_{k}\right)=P(A) .
\end{aligned}
$$

Thus $P\left(A_{i} \cup A_{j}\right)=P\left(A_{i}\right)+P\left(A_{j}\right)$. From Proposition 1 we get $\left|A_{i} \cap A_{j}\right|=0$ and $\partial^{\mathrm{M}} A_{i} \cap \partial^{\mathrm{M}} A_{j}=\emptyset\left(\bmod \mathcal{H}^{N-1}\right)$.

(iii) $\Longrightarrow$ (iv) We know that $\mathcal{H}^{N-1}$-a.e. $x \in \partial^{\mathrm{M}} A_{i}$ belongs to $A_{i}^{1 / 2}$ and to $\cap_{j \neq i} \mathbb{R}^{\mathrm{N}} \backslash$ $\partial^{\mathrm{M}} A_{j}$, hence is a point of density 0 for all sets $A_{j}$ with $j \neq i$. Let us fix a point $x$ with these properties and assume, in addition, that

$$
\lim _{r \rightarrow 0^{+}} \frac{\mathcal{H}^{N-1}\left(\cup_{j \neq i} \partial^{\mathrm{M}} A_{j} \cap B(x, r)\right)}{r^{N-1}}=0 .
$$

By (4) with $B=\cup_{j \neq i} \partial^{\mathrm{M}} A_{j}$ we know that also this additional condition is fulfilled $\mathcal{H}^{N-1}$-a.e. in $\partial^{\mathrm{M}} A_{i}$. The relative isoperimetric inequality (7) easily implies the existence of a constant $c$ such that

$|E \cap B(x, r)| \leq c r \mathcal{H}^{N-1}\left(\partial^{\mathrm{M}} E \cap B(x, r)\right) \quad$ whenever $\quad|B(x, r) \backslash E| \geq \frac{|B(x, r)|}{4}$

Hence

$$
\left|A_{j} \cap B(x, r)\right| \leq c r \mathcal{H}^{N-1}\left(\partial^{\mathrm{M}} A_{j} \cap B(x, r)\right) \quad \forall j \neq i
$$

for any $r>0$ sufficiently small, such that $\left|A_{i} \cap B(x, r)\right| \geq|B(x, r)| / 4$. Adding with respect to $j$ we obtain

$$
\lim _{r \rightarrow 0^{+}} \frac{\left|\left(A \backslash A_{i}\right) \cap B(x, r)\right|}{r^{N}} \leq c \lim _{r \rightarrow 0^{+}} \frac{\mathcal{H}^{N-1}\left(\cup_{j \neq i} \partial^{\mathrm{M}} A_{j} \cap B(x, r)\right)}{r^{N-1}}=0 .
$$

Hence $x \in A^{1 / 2} \subset \partial^{\mathrm{M}} A$.

(iv) $\Longrightarrow$ (i) Since $\left(A_{i}\right)^{1 / 2} \cap\left(A_{j}\right)^{1 / 2} \subset \AA^{\mathrm{M}}$ whenever $i \neq j$ (because the sets $A_{i}$ are pairwise disjoint), we obtain that

$$
\mathcal{H}^{N-1}\left(\partial^{\mathrm{M}} A_{i} \cap \partial^{\mathrm{M}} A_{j}\right)=\mathcal{H}^{N-1}\left(\partial^{\mathrm{M}} A \cap\left(A_{i}\right)^{1 / 2} \cap\left(A_{j}\right)^{1 / 2}\right)=0
$$

hence $\sum_{i} P\left(A_{i}\right)=\sum_{i} \mathcal{H}^{N-1}\left(\partial^{\mathrm{M}} A_{i}\right) \leq \mathcal{H}^{N-1}\left(\partial^{\mathrm{M}} A\right)=P(A)$. 
The identity $\partial^{\mathrm{M}} A=\cup_{i} \partial^{\mathrm{M}} A_{i}\left(\bmod \mathcal{H}^{N-1}\right)$ follows by (ii). Since $\AA^{\mathrm{M}} \cap \partial^{\mathrm{M}} A=\emptyset$, (4) again with $B=\partial^{\mathrm{M}} A$ gives that $\mathcal{H}^{N-1}\left(B(x, r) \cap \partial^{\mathrm{M}} A\right) / r^{N-1}$ tends to 0 as $r \rightarrow 0^{+}$for $\mathcal{H}^{N-1}$-a.e. $x \in \AA^{\mathrm{M}}$, Thus, in order to prove (8) we prove the inclusion

$$
\AA^{\mathrm{M}} \backslash \bigcup_{i \in I} \AA_{i}^{\mathrm{M}} \subset\left\{x \in \mathbb{R}^{\mathrm{N}}: \limsup _{r \rightarrow 0^{+}} \frac{\mathcal{H}^{N-1}\left(B(x, r) \cap \partial^{\mathrm{M}} A\right)}{r^{N-1}}>0\right\} .
$$

Let $x \in \AA^{\mathrm{M}}$ be such that $\mathcal{H}^{N-1}\left(B(x, r) \cap \partial^{\mathrm{M}} A\right) / r^{N-1}$ tends to 0 as $r \rightarrow 0^{+}$. Let $r_{0}>0$ and $\sigma \in(0,1 / 2)$ such that $\mathcal{H}^{N-1}\left(B(x, r) \cap \partial^{\mathrm{M}} A\right) \leq \sigma \omega_{N} r^{N-1} / \eta_{N}$ for any $r \in\left(0, r_{0}\right]$. By the relative isoperimetric inequality (7) we infer

$$
\min \left\{\left|B(x, r) \cap A_{i}\right|,\left|B(x, r) \backslash A_{i}\right|\right\} \leq \sigma|B(x, r)| \quad \forall i \in I, r \in\left(0, r_{0}\right] .
$$

Since the sets $A_{i}$ are pairwise disjoint, the family

$$
\begin{aligned}
R_{i} & :=\left\{r \in\left(0, r_{0}\right]:\left|B(x, r) \cap A_{i}\right| \geq(1-\sigma)|B(x, r)|\right\}, \\
R_{\infty} & :=\left\{r \in\left(0, r_{0}\right]:\left|B(x, r) \cap A_{i}\right| \leq \sigma|B(x, r)| \forall i \in I\right\}
\end{aligned}
$$

is a partition of $\left(0, r_{0}\right]$ in relatively closed sets. Being $\left(0, r_{0}\right]$ connected, one of these sets coincides with $\left(0, r_{0}\right]$. If $\left(0, r_{0}\right]=R_{\infty}$ the relative isoperimetric inequality (7) gives

$$
|B(x, r) \cap A|=\sum_{i \in I}\left|B(x, r) \cap A_{i}\right| \leq r \eta_{N} \sum_{i \in I} \mathcal{H}^{N-1}\left(B(x, r) \cap \partial^{\mathrm{M}} A_{i}\right) \leq \sigma|B(x, r)|
$$

for any $r \in\left(0, r_{0}\right]$, which is a contradiction. If $\left(0, r_{0}\right]=R_{i}$ for some $i \in I$, then we have that $\underline{D}\left(A_{i}, x\right) \geq 1-\sigma$. Choose a sequence $\sigma_{n} \rightarrow 0+$ and $i_{n} \in \mathbb{N}$ such that $\underline{D}\left(A_{i_{n}}, x\right) \geq 1-\sigma_{n}$. Then, $i_{n}$ is constant for $n$ large enough, say $i_{n}=i$ for $n$ large enough. Thus we conclude that $D\left(A_{i}, x\right)=1$, i.e, $x \in \AA_{i}^{\mathrm{M}}$.

Remark 1 (Additional properties of partitions). Under the assumptions of the previous proposition, we remark that if $|A|=\infty$, due to the fact that the series of perimeters is convergent, there is exactly one set $A_{i}$ with infinite measure; indeed, if all of them have finite measure, from the isoperimetric inequality we get

$$
\sum_{i:\left|A_{i}\right| \leq 1}\left|A_{i}\right|^{\frac{N-1}{N}}+\sum_{i:\left|A_{i}\right| \geq 1}\left|A_{i}\right|^{\frac{N-1}{N}} \leq \gamma_{N} \sum_{i \in I} P\left(A_{i}\right)<\infty
$$

and we obtain that $\left|A_{i}\right| \geq 1$ only for finitely many $i$. Thus

$$
\infty=\sum_{i:\left|A_{i}\right| \leq 1}\left|A_{i}\right| \leq \sum_{i:\left|A_{i}\right| \leq 1}\left|A_{i}\right|^{\frac{N-1}{N}} \leq \gamma_{N} \sum_{i \in I} P\left(A_{i}\right)<\infty
$$

This contradiction proves that at least one set has infinite measure. Suppose that at least two of them, say $A_{i_{0}}, A_{i_{1}}$, have infinite measure. Again by the isoperimetric inequality we would get

$$
\min \left\{\left|A_{i_{0}}\right|^{\frac{N-1}{N}},\left|\mathbb{R}^{\mathrm{N}} \backslash A_{i_{0}}\right|^{\frac{N-1}{N}}\right\} \leq P\left(A_{i_{0}}\right) \leq \sum_{j \neq i_{0}} P\left(A_{j}\right)<\infty .
$$

However, the quantity on the left hand side is infinite since $A_{i_{1}} \subseteq \mathbb{R}^{\mathrm{N}} \backslash A_{i_{0}}$. 
We notice also that the argument used in the proof of (ii) $\Longrightarrow$ (iii) gives

$$
P\left(\bigcup_{i \in I_{1} \cup I_{2}} A_{i}\right)=P\left(\bigcup_{i \in I_{1}} A_{i}\right)+P\left(\bigcup_{i \in I_{2}} A_{i}\right)
$$

whenever $I_{1}, I_{2} \subseteq I$ are disjoint.

As a consequence of Proposition 3 with $A=E, A_{1}=F$ and $A_{2}=E \backslash F$, we obtain that characteristic functions of sets of finite perimeter $F$ are constant inside an indecomposable set $E$, provided $\chi_{F}$ has no "derivative" in $E$. This is expressed by saying that $\partial^{\mathrm{M}}(E \cap F) \subset \partial^{\mathrm{M}} E$, or equivalently that $\partial^{\mathrm{M}}(E \cap F) \cap \dot{E}^{\mathrm{M}}=\varnothing$ $\left(\bmod \mathcal{H}^{N-1}\right)$. A more general statement is presented in Remark 2.

Proposition 4. Let $E$ be an indecomposable set and let $F \subseteq E$ be a set with finite perimeter, such that $\partial^{\mathrm{M}} F \subseteq \partial^{\mathrm{M}} E\left(\bmod \mathcal{H}^{N-1}\right)$. Then either $|F|=0$ or $|E \backslash F|=0$.

Remark 2 (Constancy theorem). Since $F \subseteq E$, the assumption $\partial^{\mathrm{M}} F \subseteq \partial^{\mathrm{M}} E$ $\left(\bmod \mathcal{H}^{N-1}\right)$ in Proposition 4 is equivalent to $\mathcal{H}^{N-1}\left(\partial^{\mathrm{M}} F \cap \stackrel{\circ}{E}^{\mathrm{M}}\right)=0$. Proposition 4 is a particular case of the following result, proved by G. Dolzmann and $\mathrm{S}$. Müller in [21]: if $u \in \mathrm{BV}_{\text {loc }}\left(\mathbb{R}^{\mathrm{N}}\right)$ satisfies $|D u|\left(\mathbb{R}^{\mathrm{N}}\right)<\infty$ and $E$ is indecomposable, then

$$
|D u|\left(\stackrel{\circ}{E}^{\mathrm{M}}\right)=0 \quad \Longrightarrow \quad \exists c \in \mathbb{R}: u(x)=c \text { for a.e. } x \in E .
$$

The proof follows by the coarea formula

$$
|D u|\left(\stackrel{\circ}{E}^{\mathrm{M}}\right)=\int_{-\infty}^{\infty} \mathcal{H}^{N-1}\left(\partial^{\mathrm{M}}\{u>\lambda\} \cap \stackrel{\circ}{E}^{\mathrm{M}}\right) d \lambda
$$

noticing that Proposition 4 applies to a.e. level set $F_{\lambda}=\{u>\lambda\}$.

The main result of this section is the following decomposition theorem; a similar (see Remark 4) decomposition result for integer currents is stated in 4.2.25 of [24]. This result has also been used in G. Dolzmann and S. Müller [21] and B. Kirchheim [36] to prove Liouville type theorems for a class of partial differential inclusions; the second paper contains also an explicit proof of the decomposition theorem, based on Lyapunov convexity theorem (see also Theorem 1 in $\$ 3.4$ of Chap. 4 of [28]). The proof that we present here is new and based on a simple variational argument.

Theorem 1 (Decomposition theorem). Let $E$ be a set with finite perimeter in $\mathbb{R}^{\mathrm{N}}$. Then there exists a unique finite or countable family of pairwise disjoint indecomposable sets $\left\{E_{i}\right\}_{i \in I}$ such that $\left|E_{i}\right|>0$ and $P(E)=\sum_{i} P\left(E_{i}\right)$. Moreover

$$
\mathcal{H}^{N-1}\left(\stackrel{\circ}{E}^{\mathrm{M}} \backslash \bigcup_{i \in I} \stackrel{\circ}{E}_{i}^{\mathrm{M}}\right)=0
$$

and the $E_{i}$ 's are maximal indecomposable sets, i.e. any indecomposable set $F \subseteq E$ is contained $\left(\bmod \mathcal{H}^{N}\right)$ in some set $E_{i}$. 
Proof. (Existence) Let $\alpha \in(1, N /(N-1))$, let us define

$$
\mu(E):=\left(\int_{E} \exp \left(-|x|^{2}\right) d x\right)^{1 / \alpha}
$$

for any measurable set $E \subseteq \mathbb{R}^{\mathrm{N}}$ and let $\mathcal{P}$ be the collection of all partitions $\left\{E_{i}\right\}_{i \in \mathbb{N}}$ of $E$ such that $\left|E_{i}\right| \geq\left|E_{j}\right|$ for $i \leq j$ and $\sum_{i} P\left(E_{i}\right) \leq P(E)$. Recall that the condition $\sum_{i} P\left(E_{i}\right)<\infty$ implies that at most one set $E_{i}$ (namely $E_{0}$ ) has infinite measure (see Remark 1 ). The class $\mathcal{P}$ is not empty, since it contains $\{E, \emptyset, \emptyset, \ldots\}$.

We will prove that the problem

$$
\max \left\{\sum_{i \in \mathbb{N}} \mu\left(E_{i}\right):\left\{E_{i}\right\}_{i \in \mathbb{N}} \in \mathcal{P}\right\}
$$

has a (essentially unique) solution. Indeed, let $\left\{E_{i}^{n}\right\}_{i \in \mathbb{N}}$ be a maximizing sequence indexed by $n$; since $P\left(E_{i}^{n}\right) \leq P(E)$ by the compactness properties of sets of finite perimeter (see Sect. 2) we can assume, possibly extracting a subsequence, that $E_{i}^{n}$ locally converge in measure in $\mathbb{R}^{\mathrm{N}}$ to suitable sets $E_{i}$ as $n \rightarrow \infty$. The sets $E_{i}$ are pairwise disjoint $\left(\bmod \mathcal{H}^{N}\right)$, and the lower semicontinuity of perimeter with respect to local convergence in measure gives $\sum_{i} P\left(E_{i}\right) \leq P(E)$. In order to show that $\left\{E_{i}\right\}_{i \in \mathbb{N}} \in \mathcal{P}$ we have to prove that $\left|E \backslash \cup_{i} E_{i}\right|=0$. To this aim, we first prove that

$$
\lim _{p \rightarrow \infty} \limsup _{n \rightarrow \infty} \sum_{i=p}^{\infty} \mu\left(E_{i}^{n}\right)=0 .
$$

First, we notice that the isoperimetric inequality and the subadditivity of perimeter give

$$
p^{\frac{N-1}{N}}\left|E_{p}^{n}\right|^{\frac{N-1}{N}} \leq\left|\bigcup_{i=1}^{p} E_{i}^{n}\right|^{\frac{N-1}{N}} \leq \gamma_{N} \sum_{i=1}^{p} P\left(E_{i}^{n}\right) \leq \gamma_{N} P(E)
$$

for any $p \geq 1$ because $i \mapsto\left|E_{i}^{n}\right|$ is decreasing. Therefore

$$
\begin{aligned}
\sum_{i=p}^{\infty} \mu\left(E_{i}^{n}\right) & \leq \sum_{i=p}^{\infty}\left|E_{i}^{n}\right|^{1 / \alpha} \leq \frac{\left[\gamma_{N} P(E)\right]^{\frac{N}{\alpha(N-1)}}-1}{p^{\frac{1}{\alpha}-\frac{(N-1)}{N}}} \sum_{i=p}^{\infty}\left|E_{i}^{n}\right|^{\frac{N-1}{N}} \\
& \leq \frac{\left[\gamma_{N} P(E)\right]^{\frac{N}{\alpha(N-1)}}}{p^{\frac{1}{\alpha}-\frac{(N-1)}{N}}}
\end{aligned}
$$

proving (12).

Since $\alpha>1$, (12) also holds with $\left[\mu\left(E_{i}\right)\right]^{\alpha}$ in place of $\mu\left(E_{i}\right)$, and since $\mu\left(E_{i}^{n}\right) \rightarrow \mu\left(E_{i}\right)$ as $n \rightarrow \infty$ for any $i \in \mathbb{N}$, this implies

$$
\sum_{i \in \mathbb{N}}\left[\mu\left(E_{i}\right)\right]^{\alpha}=\lim _{n \rightarrow \infty} \sum_{i \in \mathbb{N}}\left[\mu\left(E_{i}^{n}\right)\right]^{\alpha}=[\mu(E)]^{\alpha} .
$$


By the definition of $\mu$, this proves that

$$
\int_{E \backslash \cup_{i} E_{i}} \exp \left(-|x|^{2}\right) d x=0
$$

and hence that $\left|E \backslash \cup_{i} E_{i}\right|=0$. Moreover, using (12) again we obtain

$$
\lim _{n \rightarrow \infty} \sum_{i \in \mathbb{N}} \mu\left(E_{i}^{n}\right)=\sum_{i \in \mathbb{N}} \mu\left(E_{i}\right)
$$

hence $\left\{E_{i}\right\}_{i \in \mathbb{N}}$ is maximizing. If $\left\{E_{i}\right\}_{i \in \mathbb{N}}$ is any maximizing partition, then any $E_{i}$ is clearly indecomposable, because $\mu(A)+\mu(B)>\mu(A \cup B)$ whenever $\mu(A)$ and $\mu(B)$ are strictly positive.

(Uniqueness) Let $\left(E_{i}\right)$ be a maximizing partition and let $F$ be an indecomposable set with $|F|>0$. Since $F \subseteq E$ there exists $i \in I$ such that $\left|F \cap E_{i}\right|>0$. We will prove that $F \subseteq E_{i}\left(\bmod \overline{\mathcal{H}}^{N}\right)$. Since $F$ is indecomposable, to this aim it suffices to prove that $P\left(F \backslash E_{i}\right)+P\left(F \cap E_{i}\right)=P(F)$, or equivalently that

$$
\left(F \cap E_{i}\right)^{1 / 2} \cap\left(F \backslash E_{i}\right)^{1 / 2}=\emptyset \quad\left(\bmod \mathcal{H}^{N-1}\right) .
$$

Using Proposition 3 we obtain that $\partial^{\mathrm{M}} E_{i} \subset \partial^{\mathrm{M}} E\left(\bmod \mathcal{H}^{N-1}\right)$ and $P(E)=$ $P\left(E \backslash E_{i}\right)+P\left(E_{i}\right)$. In turn, by Proposition 1 , this gives $\partial^{\mathrm{M}} E_{i} \cap \partial^{\mathrm{M}}\left(E \backslash E_{i}\right)=\emptyset$ $\left(\bmod \mathcal{H}^{N-1}\right)$. Hence, $(13)$ would be proved by the inclusion

$$
\left(F \cap E_{i}\right)^{1 / 2} \cap\left(F \backslash E_{i}\right)^{1 / 2} \subset E_{i}^{1 / 2} \cap\left(E \backslash E_{i}\right)^{1 / 2} .
$$

Any point $x$ in the set on the left side clearly belongs to $\stackrel{\circ}{F}^{\mathrm{M}}$ and hence to $\stackrel{\circ}{E}^{\mathrm{M}}$; taking this fact into account, it suffices to prove that $x \in E_{i}^{1 / 2}$, and since $x \in\left(F \cap E_{i}\right)^{1 / 2}$ this easily follows by the fact that $E_{i} \backslash\left(F \cap E_{i}\right)$ is contained in the complement of $F$. This proves the maximal character of $E_{i}$.

Finally, if $\left\{E_{i}\right\}_{i \in I}$ and $\left\{F_{j}\right\}_{j \in J}$ are two maximizing partitions, we know that any $E_{i}$ is contained in one (and only one) $F_{j}$ and any $F_{j}$ is contained in one (and only one) $E_{i}$. Equation (11) follows by (8).

Definition 1 ( $M$-connected components). In view of the previous theorem, we call the sets $E_{i}$ the $M$-connected components of $E$ and denote this family by $\mathcal{C C}^{\mathrm{M}}(E)$; we always choose the index set I as an interval of $\mathbb{N}$, with $0 \in I$.

Notice that $\mathcal{C C}^{\mathrm{M}}(E)=\emptyset$ whenever $E$ is Lebesgue negligible and that Proposition 3 gives

$$
\partial^{\mathrm{M}} F \subset \partial^{\mathrm{M}} E \quad\left(\bmod \mathcal{H}^{N-1}\right) \quad \text { for any } F \in \mathcal{C C}^{\mathrm{M}}(E) .
$$

By (8), for $\mathcal{H}^{N-1}$-a.e. $x \in \stackrel{\circ}{E}^{\mathrm{M}}$ it also makes sense to talk about the $M$-connected component of $E$ containing $x$, namely the unique set $F \in \mathcal{C C}^{\mathrm{M}}(E)$ such that $x \in \stackrel{\circ}{F}^{\mathrm{M}}$. The necessity to exclude an exceptional $\mathcal{H}^{N-1}$-negligible set is shown by the following example. 
Example 1. Let $K \subseteq\left\{x_{2}=0\right\} \subseteq \mathbb{R}^{2}$ be a compact and $\mathcal{H}^{1}$-negligible set and let $\phi\left(x_{1}\right)=\operatorname{dist}^{2}\left(x_{1}, K\right)$. Then, the set

$$
E:=\left\{x=\left(x_{1}, x_{2}\right) \in \mathbb{R}^{2}: x_{2}<0 \text { or } x_{2}>\phi\left(x_{1}\right)\right\}
$$

has two $M$-connected components $E_{1}, E_{2}$ and it is easy to check that $K \subset \stackrel{\circ}{E}^{\mathrm{M}} \backslash$ $\left({\stackrel{\circ}{E_{1}}}^{\mathrm{M}} \cup{\dot{E_{2}}}^{\mathrm{M}}\right)$.

In the following theorem we prove that $\mathcal{C C}^{\mathrm{M}}(A)$ coincides with the family of connected components of $A$ for any sufficiently regular open set $A$; we prove in Remark 3 that for any Lipschitz function $u: \mathbb{R}^{\mathrm{N}} \rightarrow \mathbb{R}$ almost every upper level set $\{u>\lambda\}$ has this (weak) regularity property. In general an open indecomposable set needs not be connected: for instance a disk without a diameter is disconnected but indecomposable. Example 2 shows in addition that an indecomposable set need not be equivalent $\left(\bmod \mathcal{H}^{N}\right)$ to an open connected set.

Theorem 2. Let $A \subseteq \mathbb{R}^{\mathrm{N}}$ be an open set such that $\mathcal{H}^{N-1}(\partial A)=\mathcal{H}^{N-1}\left(\partial^{\mathrm{M}} A\right)$. Then $\mathcal{C C}^{\mathrm{M}}(A)$ coincides with the family of connected components of $A$.

Proof. The connected components $\left\{A_{i}\right\}_{i \in I}$ of $A$ are pairwise disjoint, indecomposable by Proposition 2 and satisfy

$$
\partial^{\mathrm{M}} A_{i} \subseteq \partial A \subseteq \partial^{\mathrm{M}} A \quad\left(\bmod \mathcal{H}^{N-1}\right) \quad \forall i \in I
$$

By Proposition 3 we obtain that $\sum_{i} P\left(A_{i}\right) \leq P(A)$. Hence, Theorem 1 implies that $A_{i}$ are the $M$-connected components of $A$.

Example 2. Let $K \subset(0,1)$ be a compact set with empty interior and strictly positive measure and let $I_{i}=\left(a_{i}, b_{i}\right)$ be the connected components of $(0,1) \backslash K$, indexed by $i \in I$, and let $c_{i}$ be the central point of $I_{i}$. We define

$$
A=(0,1) \times\left(-\frac{1}{2}, \frac{1}{2}\right) \backslash \overline{\bigcup_{i \in I} B_{i}} \subseteq \mathbb{R}^{2},
$$

where $B_{i}$ are closed balls centered at $\left(c_{i}, 0\right)$ with radius $b_{i}-c_{i}$ (see Fig. 1 ). Then, since $K$ has empty interior it is easy to check that

$$
\overline{\bigcup_{i \in I} B_{i}}=\bigcup_{i \in I} B_{i} \cup[0,1] \times\{0\},
$$

hence $A$ is disconnected by the two open sets $A_{1}=A \cap\left\{x_{2}>0\right\}$ and $A_{2}=$ $A \cap\left\{x_{2}<0\right\}$. On the other hand, we claim that $A$ is indecomposable: indeed, since $A_{i}$ are connected open sets, they are also indecomposable and hence are contained in $M$-connected components of $A$. Thus, if $A$ were decomposable we would get $\mathcal{C C}^{\mathrm{M}}(A)=\left\{A_{1}, A_{2}\right\}$, and this contradicts the fact that $\partial^{\mathrm{M}} A_{1}$ and $\partial^{\mathrm{M}} A_{2}$ intersect on $K \times\{0\}$, a set with strictly positive $\mathcal{H}^{1}$ measure. 


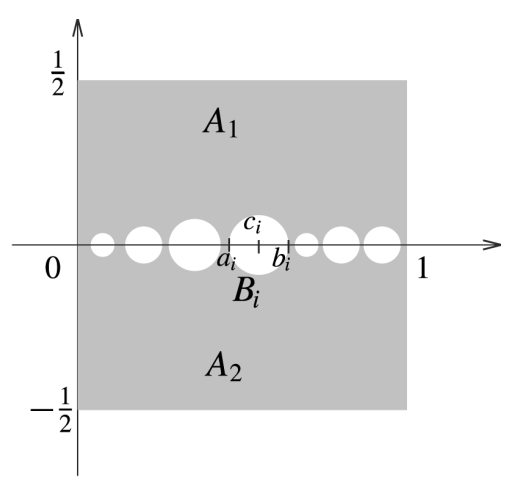

Fig. 1. An example illustrating the fact that the $M$-connected components of an open set do not coincide in general with the classical connected components

Remark 3. For any Lipschitz function $u: \mathbb{R}^{\mathrm{N}} \rightarrow \mathbb{R}$ the set $\{u>\lambda\}$ satisfies the assumption of Theorem 2 for a.e. $\lambda \in \mathbb{R}$. Indeed, let $\Omega \subseteq \mathbb{R}^{\mathrm{N}}$ be a bounded open set; by applying both the coarea formula for BV functions (see Sect. 3) and the coarea formula for Lipschitz functions (see for instance [22]) we get

$$
\begin{aligned}
\int_{-\infty}^{+\infty} \mathcal{H}^{N-1}(\Omega \cap\{u=\lambda\}) d \lambda & =\int_{\Omega}|\nabla u| d x \\
& =\int_{-\infty}^{+\infty} \mathcal{H}^{N-1}\left(\Omega \cap \partial^{\mathrm{M}}\{u>\lambda\}\right) d \lambda<\infty
\end{aligned}
$$

Since $\partial\{u>\lambda\} \subset\{u=\lambda\}$ for any $\lambda \in \mathbb{R}$, this proves that

$$
\mathcal{H}^{N-1}\left(\Omega \cap \partial^{\mathrm{M}}\{u>\lambda\}\right)=\mathcal{H}^{N-1}(\Omega \cap \partial\{u>\lambda\}) \quad \text { for a.e. } \lambda \in \mathbb{R} .
$$

Taking a countable family of open sets $\Omega_{h}$ whose union is $\mathbb{R}^{\mathrm{N}}$ our statement follows.

Using the decomposition theorem we can easily prove that indecomposable sets have the same stability properties of connected sets.

\section{Proposition 5 (Stability of indecomposable sets).}

(i) If $E_{1}, E_{2}$ are indecomposable and either $\left|E_{1} \cap E_{2}\right|>0$ or $\mathcal{H}^{N-1}\left(\partial^{\mathrm{M}} E_{1} \cap\right.$ $\left.\partial^{\mathrm{M}} E_{2}\right)>0$, then $E_{1} \cup E_{2}$ is indecomposable.

(ii) If $\left(E_{h}\right)$ is an increasing sequence of indecomposable sets with equibounded perimeters, then $\cup_{h} E_{h}$ is indecomposable.

Proof. (i) Let $\left\{G_{i}\right\}_{i \in I}$ be the components of $E_{1} \cup E_{2}$ and let $j_{1}, j_{2} \in I$ such that $E_{i} \subseteq G_{j_{i}}$. If $\left|E_{1} \cap E_{2}\right|>0$, since the $G_{i}$ 's are pairwise disjoint, we conclude that $j_{1}=j_{2}$, hence $\mathcal{C} \mathcal{C}^{\mathrm{M}}\left(E_{1} \cup E_{2}\right)=\left\{G_{j_{1}}\right\}$. Otherwise we conclude that $\mathcal{C C}^{\mathrm{M}}\left(E_{1} \cup E_{2}\right)$ $=\left\{E_{1}, E_{2}\right\}$, hence Proposition 1 gives $\mathcal{H}^{N-1}\left(\partial^{\mathrm{M}} E_{1} \cap \partial^{\mathrm{M}} E_{2}\right)=0$. The proof of (ii) is analogous. 
We conclude this section with the analysis of the relation between indecomposability of a set and the indecomposability of its boundary, in the sense of $\mathrm{H}$. Federer. To do this, we will adopt the notations of [24]; since this comparison is not really needed in the following, the reader unfamiliar with the theory of currents can simply skip this part.

Remark 4 (Indecomposability in the sense of Federer). Let us consider the collection of all $k$-dimensional normal integer currents $T$, denoted by $\mathbf{I}^{k}\left(\mathbb{R}^{\mathrm{N}}\right)$. A current $T \in \mathbf{I}^{k}\left(\mathbb{R}^{\mathrm{N}}\right)$ is said to be indecomposable if $T=T_{1}+T_{2}, M(T)=M\left(T_{1}\right)+M\left(T_{2}\right)$ and $M(\partial T)=M\left(\partial T_{1}\right)+M\left(\partial T_{2}\right)$ with $T_{i} \in \mathbf{I}^{k}\left(\mathbb{R}^{\mathrm{N}}\right)$ implies that either $T_{1}$ or $T_{2}$ are zero (here $M$ denotes the mass, i.e. the area with multiplicities). Using Proposition 1 , it is easy to show that the canonical $N$-current $\llbracket E \rrbracket \in \mathbf{I}^{N}\left(\mathbb{R}^{N}\right)$ associated to a set of finite perimeter $E$ is indecomposable if and only if $E$ is indecomposable; however, notice that the indecomposability of $E$ is not equivalent to the indecomposability of its boundary (it suffices to consider as $E$ an annulus).

In 4.2.25 of [24] it is stated that any $T \in \mathbf{I}^{k}\left(\mathbb{R}^{\mathrm{N}}\right)$ admits a decomposition in finitely or countably many indecomposable components; the proof (suggested and not explicitly given) again relies on the isoperimetric inequality and could be obtained mimicking our one, i.e. maximizing $\sum_{i}\left[M\left(T_{i}\right)\right]^{1 / \alpha}$, with $\alpha \in(1, k /(k-1))$, among all possible decompositions $T_{i}$. However, no uniqueness theorem for the decomposition holds for $k<N$.

\section{Holes, saturation, simple sets}

In this section we see how the decomposition theorem leads to reasonably good definitions of "hole" and "saturation" for a set of finite perimeter. These concepts will be used in the next section to recover a canonical decomposition of the measure theoretic boundary.

Definition 2 (Holes, saturation). Let $E$ be an indecomposable set. We call hole of $E$ any $M$-connected component of $\mathbb{R}^{\mathrm{N}} \backslash E$ with finite measure. We define the saturation of $E$, denoted by $\operatorname{sat}(E)$, as the union of $E$ and its holes. In the general case when $E$ has finite perimeter, we define

$$
\operatorname{sat}(E):=\bigcup_{i \in I} \operatorname{sat}\left(E_{i}\right) \quad \text { where } \quad \mathcal{C C}^{\mathrm{M}}(E)=\left\{E_{i}\right\}_{i \in I}
$$

We call $E$ saturated if $\operatorname{sat}(E)=E$.

We first investigate the saturation operator on indecomposable sets and later we extend this analysis to any set of finite perimeter.

Proposition 6. Let $E \subseteq \mathbb{R}^{\mathrm{N}}$ be an indecomposable set.

(i) Any hole of $E$ is saturated.

(ii) $\operatorname{sat}(E)$ is indecomposable, saturated, $\partial^{\mathrm{M}} \operatorname{sat}(E) \subset \partial^{\mathrm{M}} E\left(\bmod \mathcal{H}^{N-1}\right)$ and $\operatorname{sat}(E)$ has finite measure if $|E|<\infty$. In particular $P(\operatorname{sat}(E)) \leq P(E)$.

(iii) If $E \subset \operatorname{sat}(F)$ then $\operatorname{sat}(E) \subset \operatorname{sat}(F)$. 
(iv) If $F$ is indecomposable and $|F \cap E|=0$, then the sets $\operatorname{sat}(E)$, $\operatorname{sat}(F)$ are either one a subset of the other, or are disjoint.

Proof. (i) Let $Y$ be an hole of $E$ and let $\mathcal{C} \mathcal{C}^{\mathrm{M}}\left(\mathbb{R}^{\mathrm{N}} \backslash E\right)=\{Y\} \cup\left\{Y_{j}\right\}_{j \in J}$. Then

$$
\mathbb{R}^{\mathrm{N}} \backslash Y=E \cup \bigcup_{j \in J} Y_{j}
$$

Since by (15) $\partial^{\mathrm{M}} Y_{j} \subset \partial^{\mathrm{M}} E\left(\bmod \mathcal{H}^{N-1}\right)$, Proposition 5(i) gives that $E \cup \cup_{j \in J^{\prime}} Y_{j^{\prime}}$ is indecomposable for any finite set $J^{\prime} \subseteq J$. By Proposition 5(ii) we conclude that $\mathbb{R}^{\mathrm{N}} \backslash Y$ is indecomposable, i.e. $Y$ has no hole.

(ii) We can assume with no loss of generality that $|E|<\infty$ (otherwise $\operatorname{sat}(E)=$ $\mathbb{R}^{\mathrm{N}}$ ) and denote by $Y_{0}$ the $M$-connected component of $\mathbb{R}^{\mathrm{N}} \backslash E$ with infinite measure. The proof that $\operatorname{sat}(E)$ is indecomposable relies, as the one of (i), on Proposition 5. Since $\operatorname{sat}(E)=\mathbb{R}^{\mathrm{N}} \backslash Y_{0}$, sat $(E)$ is saturated. Finally, the inclusion $\partial^{\mathrm{M}} \operatorname{sat}(E) \subset \partial^{\mathrm{M}} E$ $\left(\bmod \mathcal{H}^{N-1}\right)$ follows by $(15)$.

(iii) Without loss of generality we can assume that $|F|<\infty$. Then $\mathbb{R}^{\mathrm{N}} \backslash \operatorname{sat}(F)$, being indecomposable, is contained in a $M$-connected component of $\mathbb{R}^{\mathrm{N}} \backslash E$; since $\left|\mathbb{R}^{\mathrm{N}} \backslash \operatorname{sat}(F)\right|=\infty$ we conclude that $\mathbb{R}^{\mathrm{N}} \backslash \operatorname{sat}(F) \subseteq \mathbb{R}^{\mathrm{N}} \backslash \operatorname{sat}(E)$.

(iv) We may assume that both sets are nontrivial and that their saturations are not $\mathbb{R}^{\mathrm{N}}$; we denote by $E_{0}, F_{0}$ the $M$-connected components with infinite measure of $\mathbb{R}^{\mathrm{N}} \backslash E, \mathbb{R}^{\mathrm{N}} \backslash F$ respectively. Since $|E \cap F|=0$, we know that $E$ is contained either in a hole of $F$ or in $F_{0}$. If $E$ is contained in a hole of $F$, then $E \subseteq \operatorname{sat}(F)$ and therefore $\operatorname{sat}(E) \subseteq \operatorname{sat}(F)$. Analogously, if $E \subseteq F_{0}$ and $F$ is contained in a hole of $E$, then $\operatorname{sat}(F) \subseteq \operatorname{sat}(E)$. Thus we may assume that $E \subseteq F_{0}$ and $F \subseteq E_{0}$, hence

$$
|E \cap \operatorname{sat}(F)|=0 \quad \text { and } \quad|F \cap \operatorname{sat}(E)|=0 .
$$

Under this assumption, let us prove that $|\operatorname{sat}(E) \cap \operatorname{sat}(F)|=0$. To this aim, by (16), it suffices to show that $|Y \cap \operatorname{sat}(F)|=0$ for any hole $Y$ of $E$. Since, by (16) again, $Y \subset \mathbb{R}^{\mathrm{N}} \backslash F, Y$ is contained in a $M$-connected component of $\mathbb{R}^{\mathrm{N}} \backslash F$. If $Y \subseteq F_{0}$ the proof is finished, otherwise $Y \subseteq Y^{\prime}$ for some hole $Y^{\prime}$ of $F$ which, in turn, is contained in some $M$-connected component $Y^{\prime \prime}$ of $\mathbb{R}^{\mathrm{N}} \backslash E$. But then $Y^{\prime \prime}=Y$ and therefore $Y^{\prime}=Y$. Since by $(15) \partial^{\mathrm{M}} Y \subset\left(\partial^{\mathrm{M}} E \cap \partial^{\mathrm{M}} F\right)\left(\bmod \mathcal{H}^{N-1}\right)$, if we choose $x \in Y^{1 / 2} \cap E^{1 / 2} \cap F^{1 / 2}$ we find that $|E \cap F \cap B(x, r)|>0$ for $r>0$ sufficiently small; this contradiction proves that $Y \subseteq F_{0}$.

Definition 3 (Simple sets). Any indecomposable and saturated subset of $\mathbb{R}^{\mathrm{N}}$ will be called simple.

Notice that the only simple set with infinite measure is $\mathbb{R}^{\mathrm{N}}$ and that, according to Proposition 6, the saturation of any indecomposable set $E$ is simple (actually, the smallest simple set containing $E$ ). In order to show coincidence with simple sets we will often use the following proposition.

Proposition 7. Let $E$ be a simple set and let $F \subseteq \mathbb{R}^{\mathrm{N}}$ be a set with finite perimeter, such that $\partial^{\mathrm{M}} F \subseteq \partial^{\mathrm{M}} E\left(\bmod \mathcal{H}^{N-1}\right)$ and $|F| \in(0, \infty)$. Then $F=E$. 
Proof. It suffices to apply Proposition 4 to $E$ and $F \cap E$ and to $\mathbb{R}^{\mathrm{N}} \backslash E$ and $F \backslash E$.

The property stated in Proposition 7 actually characterizes simple sets with finite measure; we also give another nice characterization of these sets due to W.H. Fleming.

Proposition 8 (Characterizations of simple sets). Let $E \subseteq \mathbb{R}^{\mathrm{N}}$ be a set with finite perimeter such that $|E| \in(0, \infty)$. Then, the following conditions are equivalent:

(i) E is simple;

(ii) E satisfies the property stated in Proposition 7;

(iii) $\chi_{E} / P(E)$ is an extreme point of the convex set

$$
\left\{u \in \mathrm{BV}\left(\mathbb{R}^{\mathrm{N}}\right):|D u|\left(\mathbb{R}^{\mathrm{N}}\right) \leq 1\right\} .
$$

Proof. The implication (i) $\Longrightarrow$ (ii) is Proposition 7. The converse implication can be proved by noticing that any hole $Y$ of $E$ satisfies $\partial^{\mathrm{M}} Y \subset \partial^{\mathrm{M}} E\left(\bmod \mathcal{H}^{N-1}\right)$ and hence coincides with $E$. This contradiction proves that $E$ has no hole, i.e. $\operatorname{sat}(E)=E$. The equivalence of (ii) and (iii) is proved (in a slightly different setting, since a bound on the supports of the functions is required) in [25].

We close this section with the following result, showing that the $M$-connected components of $\operatorname{sat}(E)$ are contained in the family of saturations of $M$-connected components of $E$.

Theorem 3 (M-connected components and saturation). Let $E \subseteq \mathbb{R}^{\mathrm{N}}$ be a set of finite perimeter. Then

$$
\mathcal{C C}^{\mathrm{M}}(\operatorname{sat}(E)) \subset\left\{\operatorname{sat}\left(E_{i}\right)\right\}_{i \in I} \quad \text { where } \quad \mathcal{C C}^{\mathrm{M}}(E)=\left\{E_{i}\right\}_{i \in I}
$$

In particular $\partial^{\mathrm{M}} \operatorname{sat}(E) \subset \partial^{\mathrm{M}} E\left(\bmod \mathcal{H}^{N-1}\right)$ and the operator sat is idempotent, i.e. $\operatorname{sat}(\operatorname{sat}(E))=\operatorname{sat}(E)$.

Proof. Let $\mathcal{C C}^{\mathrm{M}}(E)=\left\{E_{i}\right\}_{i \in I}$ and assume with no loss of generality that $|E|<\infty$; we know by Proposition 6 and the isoperimetric inequality that $\operatorname{sat}\left(E_{i}\right)$ are indecomposable sets satisfying the conditions of Lemma 2 below. Hence, $\left\{\operatorname{sat}\left(E_{j}\right)\right\}_{j \in J}$ provides a disjoint partition of $\operatorname{sat}(E)$ in indecomposable sets.

Finally, (15) and Proposition 6(ii) give

$$
\partial^{\mathrm{M}} \operatorname{sat}(E) \subset \bigcup_{j \in J} \partial^{\mathrm{M}} \operatorname{sat}\left(E_{j}\right) \subset \bigcup_{i \in I} \partial^{\mathrm{M}} E_{i} \subset \partial^{\mathrm{M}} E
$$

where all inclusions are understood $\left(\bmod \mathcal{H}^{N-1}\right)$.

Lemma 2. Let $I \subset \mathbb{N}$ and let $\left\{F_{i}\right\}_{i \in I}$ be a family of sets such that for any $i, j \in I$ either $F_{i} \subseteq F_{j}$ or $F_{j} \subseteq F_{i}$ or $F_{i} \cap F_{j}=\emptyset\left(\bmod \mathcal{H}^{N}\right)$. Then, assuming that $\left|F_{i}\right| \rightarrow 0$ as $i \rightarrow \infty$ if $I$ is countable, there exists $J \subseteq I$ such that $\left\{F_{j}\right\}_{j \in J}$ are pairwise disjoint $\left(\bmod \mathcal{H}^{N}\right)$ and $\left|\cup_{i} F_{i} \backslash \cup_{j} F_{j}\right|=0$.

Proof. It suffices to consider the partial order $i \preceq j$ if $\left|F_{j} \backslash F_{i}\right|=0$ and to take its maximal elements. If $I$ is countable, the existence of maximal elements follows easily by the assumption that $\left|F_{i}\right| \rightarrow 0$ as $i \rightarrow \infty$. 


\section{Description of sets of finite perimeter in terms of their boundary}

In general a decomposition in $M$-connected components does not lead directly to a canonical decomposition of the boundary. The aim of this section is to show that this goal can be achieved by looking to the saturations and to the holes of all $M$-connected components of $E$.

Definition 4 (Exterior). If $E \subseteq \mathbb{R}^{\mathrm{N}}$ has finite perimeter and $|E|<\infty$, we call exterior of $E$ the unique $\left(\bmod \overline{\mathcal{H}}^{N}\right) M$-component of $\mathbb{R}^{\mathrm{N}} \backslash E$ with infinite measure. The exterior of $E$ will be denoted by $\operatorname{ext}(E)$.

Notice that the notion of exterior makes sense only if $|E|<\infty$, due to the fact that $\mathbb{R}^{\mathrm{N}} \backslash E$ has finite measure if $P(E)<\infty$ and $|E|=\infty$.

Definition 5 (Jordan boundary). We say that a set $J$ is a Jordan boundary if there is a simple set $E$ such that $J=\partial^{\mathrm{M}} E\left(\bmod \mathcal{H}^{N-1}\right)$.

By Proposition 7, the simple set $E$ associated to a Jordan boundary $J$ is unique. In this sense, $J$ can also be thought as an oriented set, with the orientation induced by the generalized inner normal to $E$. Our terminology is motivated by the results of the following section concerning sets in the plane, see in particular Theorem 7. We shall write $\operatorname{int}(J)=E$ and $\operatorname{ext}(J)=\mathbb{R}^{\mathrm{N}} \backslash E$; notice that $\operatorname{ext}(J)=\operatorname{ext}(E)$.

Proposition 9. Let $E$ be indecomposable and let $\left\{Y_{i}\right\}_{i \in I}$ be its holes. Then

$$
E=\operatorname{sat}(E) \backslash \bigcup_{i \in I} Y_{i}=\operatorname{sat}(E) \cap \bigcap_{i \in I} \operatorname{ext}\left(Y_{i}\right)
$$

and

$$
P(E)=P(\operatorname{sat}(E))+\sum_{i \in I} P\left(Y_{i}\right)
$$

Conversely, let $F$ be simple and let $\left\{G_{i}\right\}_{i \in I}$ be indecomposable sets such that

$$
E=F \backslash \bigcup_{i \in I} G_{i}
$$

and

$$
P(E)=P(F)+\sum_{i \in I} P\left(G_{i}\right)
$$

Then $F=\operatorname{sat}(E)$ and $\left\{G_{i}\right\}_{i \in I}$ are the holes of $E$.

Proof. The first equality in (17) is a consequence of Definition 2. The second identity is a consequence of Proposition 6(i). In order to prove (18) we recall that the perimeter and the measure theoretic boundary are invariant under complement and notice that

$$
\mathbb{R}^{\mathrm{N}} \backslash E=\left(\mathbb{R}^{\mathrm{N}} \backslash \operatorname{sat}(E)\right) \cup \bigcup_{i \in I} Y_{i}
$$


Since both $\partial^{\mathrm{M}} \operatorname{sat}(E)$ and $\partial^{\mathrm{M}} Y_{i}$ are contained in $\partial^{\mathrm{M}} E$ up to $\mathcal{H}^{N-1}$-negligible sets, by Proposition 3 we infer (18).

Let us now prove the uniqueness of the decomposition given in (17). For that, let $F$ be simple and let $\left\{G_{i}\right\}_{i \in I}$ be indecomposable sets satisfying (19) and (20). Assume first that $|E|<\infty$, set $G_{\infty}=\mathbb{R}^{\mathrm{N}} \backslash F$ and observe that

$$
\mathbb{R}^{\mathrm{N}} \backslash E=\bigcup_{i \in I^{\prime}} G_{i}
$$

with $I^{\prime}=I \cup\{\infty\}$. Then, Proposition 3 gives that $\left\{G_{i}\right\}_{i \in I^{\prime}}$ are pairwise disjoint and $\partial^{\mathrm{M}} G_{i} \cap \partial^{\mathrm{M}} G_{j}=\varnothing\left(\bmod \mathcal{H}^{N-1}\right)$ whenever $i \neq j$.

Note that $G_{\infty}$ is indecomposable, since $F$ is a simple set. Thus $\left\{G_{i}\right\}_{i \in I^{\prime}}$ is a partition of $\mathbb{R}^{\mathrm{N}} \backslash E$ into indecomposable sets satisfying (20). By the uniqueness of the decomposition of $\mathbb{R}^{\mathrm{N}} \backslash E$ in $M$-connected components we conclude that $G_{\infty}=\mathbb{R}^{\mathrm{N}} \backslash \operatorname{sat}(E)$ (i.e. $\left.F=\operatorname{sat}(E)\right)$ and $\left\{G_{i}\right\}_{i \in I}$ coincides with the family of holes of $E$. In case that $E$ has infinite measure, $\mathbb{R}^{\mathrm{N}}=\operatorname{sat}(E) \subseteq \operatorname{sat}(F)=F$, i.e. $F=\mathbb{R}^{\mathrm{N}}$ and the proof follows the same steps of the previous one.

In order to simplify the following statements we enlarge the class of Jordan boundaries by introducing a formal Jordan boundary $J_{\infty}$ whose interior is $\mathbb{R}^{\mathrm{N}}$ and a formal Jordan boundary $J_{o}$ whose interior is empty; we also set $\mathcal{H}^{N-1}\left(J_{\infty}\right)=$ $\mathcal{H}^{N-1}\left(J_{o}\right)=0$ and denote by $\mathcal{S}$ this extended class of Jordan boundaries. In this way we are able to consider at the same time sets with finite and infinite measure and we can always assume that the list of components (or holes of the components) is infinite, possibly adding to it infinitely many $\operatorname{int}\left(J_{o}\right)$.

In the following theorem we describe $\partial^{\mathrm{M}} E$ by a collection of "external Jordan boundaries" $J_{i}^{+}$and "internal Jordan boundaries" $J_{i}^{-}$satisfying some inclusion properties; these properties provide an axiomatic characterization of them. However, we emphasize (see Fig. 2 in Sect. 7) that in general this description is not invariant under complementation, i.e. the external (internal) boundaries of a set are not the internal (external) boundaries of the complement; for this reason we give a different definition of these concepts the next section.

Theorem 4 (Decomposition of $\partial^{\mathrm{M}} E$ in Jordan boundaries). Let $E \subseteq \mathbb{R}^{\mathrm{N}}$ be a set of finite perimeter. Then, there is a unique decomposition of $\partial^{\mathrm{M}} E$ into Jordan boundaries $\left\{J_{i}^{+}, J_{k}^{-}: i, k \in \mathbb{N}\right\} \subseteq \mathcal{S}$, such that

(i) Given $\operatorname{int}\left(J_{i}^{+}\right), \operatorname{int}\left(J_{k}^{+}\right), i \neq k$, they are either disjoint or one is contained in the other; given $\operatorname{int}\left(J_{i}^{-}\right), \operatorname{int}\left(J_{k}^{-}\right), i \neq k$, they are either disjoint or one is contained in the other. Each $\operatorname{int}\left(J_{i}^{-}\right)$is contained in one of the $\operatorname{int}\left(J_{k}^{+}\right)$.

(ii) $P(E)=\sum_{i} \mathcal{H}^{N-1}\left(J_{i}^{+}\right)+\sum_{k} \mathcal{H}^{N-1}\left(J_{k}^{-}\right)$.

(iii) If $\operatorname{int}\left(J_{i}^{+}\right) \subseteq \operatorname{int}\left(J_{j}^{+}\right), i \neq j$, then there is some Jordan boundary $J_{k}^{-}$such that $\operatorname{int}\left(J_{i}^{+}\right) \subseteq \operatorname{int}\left(J_{k}^{-}\right) \subseteq \operatorname{int}\left(J_{j}^{+}\right)$. Similarly, if $\operatorname{int}\left(J_{i}^{-}\right) \subseteq \operatorname{int}\left(J_{j}^{-}\right), i \neq j$, then there is some Jordan boundary $J_{k}^{+} \operatorname{such}$ that $\operatorname{int}\left(J_{i}^{-}\right) \subseteq \operatorname{int}\left(J_{k}^{+}\right) \subseteq \operatorname{int}\left(J_{j}^{-}\right)$.

(iv) Setting $L_{j}=\left\{i: \operatorname{int}\left(J_{i}^{-}\right) \subseteq \operatorname{int}\left(J_{j}^{+}\right)\right\}$, the sets $Y_{j}=\operatorname{int}\left(J_{j}^{+}\right) \backslash \cup_{i \in L_{j}} \operatorname{int}\left(J_{i}^{-}\right)$ are pairwise disjoint, indecomposable and $E=\cup_{j} Y_{j}$. 
Proof. (Existence) Let $Y_{i}$ be the $M$-connected components of $E$. According to Proposition 9, let $J_{i}^{+}=\partial^{\mathrm{M}} \operatorname{sat}\left(Y_{i}\right)$ be the external Jordan boundary of $Y_{i}$ and let $J_{i, n}^{-}, n=1,2, \ldots$, be the family of the internal Jordan boundaries of $Y_{i}$, given by the boundaries of the holes of $Y_{i}$. Taking into account Proposition 6 and the fact that holes are saturated, we obtain that (i) is satisfied.

Using (18) we immediately obtain (ii). To prove (iii), suppose that $\operatorname{int}\left(J_{i}^{+}\right) \subseteq$ $\operatorname{int}\left(J_{j}^{+}\right)$, with $i \neq j$. Since $\left|Y_{i} \cap Y_{j}\right|=0, Y_{i}$ is contained in a hole of $Y_{j}$. Then there is some Jordan boundary $J_{j, k}^{-}$such that $\operatorname{int}\left(J_{i}^{+}\right) \subseteq \operatorname{int}\left(J_{j, k}^{-}\right) \subseteq \operatorname{int}\left(J_{j}^{+}\right)$. The other statement included in (iii) follows from the observation that two different holes of the same $M$-connected component are disjoint. To prove (iv) we observe that

$$
\begin{aligned}
Y_{j} & =\operatorname{int}\left(J_{j}^{+}\right) \backslash\left\{\operatorname{int}\left(J_{j, n}^{-}\right): n \in \mathbb{N}\right\} \\
& =\operatorname{int}\left(J_{j}^{+}\right) \backslash\left\{\operatorname{int}\left(J_{i, n}^{-}\right): \operatorname{int}\left(J_{i, n}^{-}\right) \subseteq \operatorname{int}\left(J_{j}^{+}\right)\right\}
\end{aligned}
$$

because any hole $\operatorname{int}\left(J_{i, n}^{-}\right)$of $Y_{i}$ contained in $\operatorname{int}\left(J_{j}^{+}\right)$, being disjoint with $Y_{j}$, is contained in a hole of $Y_{j}$.

(Uniqueness) Let $C_{i}^{+}, C_{k}^{-}, i, k \in \mathbb{N}$, be a family of Jordan boundaries satisfying (i), (ii), (iii), (iv). Let $K_{j}=\operatorname{int}\left(C_{j}^{+}\right) \backslash \cup_{i \in L_{j}} \operatorname{int}\left(C_{i}^{-}\right), j \geq 0$. By assumption, the sets $K_{j}$ are indecomposable and $E=\cup_{j} K_{j}$. Let us prove that

$$
P(E)=\sum_{j=0}^{\infty} P\left(K_{j}\right) .
$$

We say that an index $i$ is $j$-maximal if $\operatorname{int}\left(C_{i}^{-}\right) \subseteq \operatorname{int}\left(C_{j}^{+}\right)$and there is no other $\operatorname{int}\left(C_{k}^{-}\right)$such that $\operatorname{int}\left(C_{i}^{-}\right) \subseteq \operatorname{int}\left(C_{k}^{-}\right) \subseteq \operatorname{int}\left(C_{j}^{+}\right)$. Analogously, we say that an index $j$ is $i$-minimal if $\operatorname{int}\left(C_{i}^{-}\right) \subseteq \operatorname{int}\left(C_{j}^{+}\right)$and there is no other $\operatorname{int}\left(C_{k}^{+}\right)$such that $\operatorname{int}\left(C_{i}^{-}\right) \subseteq \operatorname{int}\left(C_{k}^{+}\right) \subseteq \operatorname{int}\left(C_{j}^{+}\right)$.

Let $\Psi_{j}=\{i: i$ is $j$-maximal $\}$; we observe that $\operatorname{int}\left(C_{l}^{-}\right) \subseteq \operatorname{int}\left(C_{j}^{+}\right)$, then there exist a $j$-maximal index $i$ such that $\operatorname{int}\left(C_{l}^{-}\right) \subseteq \operatorname{int}\left(C_{i}^{-}\right)$and a $l$-minimal index $k$ such that $\operatorname{int}\left(C_{k}^{+}\right) \subseteq \operatorname{int}\left(C_{j}^{+}\right)$. Indeed, if there were an increasing chain of sets $\operatorname{int}\left(C_{i}^{-}\right)$, then, by the isoperimetric inequality we would get that the sum of their perimeters is infinite, a contradiction with (ii). Similarly, there is no decreasing sequence of sets $\operatorname{int}\left(C_{k}^{+}\right)$containing $\operatorname{int}\left(C_{l}^{-}\right)$. As a consequence, we obtain

$$
K_{j}=\operatorname{int}\left(C_{j}^{+}\right) \backslash \bigcup_{i \in \Psi_{j}} \operatorname{int}\left(C_{i}^{-}\right) .
$$

Now, observe that the sets $\Psi_{j}$ are a partition of $\mathbb{N}$. First we observe that they are disjoint. Indeed, let $i \in \Psi_{j} \cap \Psi_{k}, j \neq k$. Then $\operatorname{int}\left(C_{i}^{-}\right) \subseteq \operatorname{int}\left(C_{j}^{+}\right)$and $\operatorname{int}\left(C_{i}^{-}\right) \subseteq \operatorname{int}\left(C_{k}^{+}\right)$. Thus, either $\operatorname{int}\left(C_{j}^{+}\right) \subseteq \operatorname{int}\left(C_{k}^{+}\right)$, or $\operatorname{int}\left(C_{k}^{+}\right) \subseteq \operatorname{int}\left(C_{j}^{+}\right)$. If we are in the first case, then (iii) proves that the index $i$ cannot be $k$-maximal. If we are in the second case, then (iii) proves that the index $i$ cannot be $j$-maximal. Next, let $i \in \mathbb{N}$ and let $j$ such that $j$ is $i$-minimal. Then, using (iii), we have that $i$ is $j$-maximal, i.e. $i \in \Psi_{j}$. 
By Theorem 5 below we know that

$$
P\left(K_{j}\right)=\mathcal{H}^{N-1}\left(C_{j}^{+}\right)+\sum_{i \in \Psi_{j}} \mathcal{H}^{N-1}\left(C_{i}^{-}\right)
$$

Adding both sides with respect to $j$ we obtain that $P(E)=\sum_{j} P\left(K_{j}\right)$.

By the uniqueness of the decomposition of $E$ into $M$-connected components we obtain that, up to a permutation of indexes, $K_{j}=Y_{j}$ for all $j \in \mathbb{N}$. Now, the uniqueness result of Proposition 9 proves that $\operatorname{int}\left(C_{j}^{+}\right)=\operatorname{int}\left(J_{j}^{+}\right)$and that $\operatorname{int}\left(C_{i}^{-}\right)$, $i \in \Psi_{j}$, coincide with the system of holes of $Y_{j}$.

Theorem 5. Let $\left\{J_{i}^{+}, J_{k}^{-}: i, k \in \mathbb{N}\right\} \subset \mathcal{S}$ be satisfying the conditions (i), (iii) of Theorem 4 and

(ii') Each two different Jordan boundaries of the system $\left\{J_{i}^{+}, J_{k}^{-}: i, k \geq 0\right\}$ are disjoint $\left(\bmod \mathcal{H}^{N-1}\right)$.

(iv') $\sum_{i} P\left(J_{i}^{+}\right)+\sum_{k} P\left(J_{k}^{-}\right)<\infty$.

Let $E=\cup_{j} Y_{j}$, where

$$
Y_{j}:=\operatorname{int}\left(J_{j}^{+}\right) \backslash \bigcup_{i \in L_{j}} \operatorname{int}\left(J_{i}^{-}\right) .
$$

Then $E$ is a set of finite perimeter and $\partial^{\mathrm{M}} E=\cup_{i} J_{i}^{+} \cup \cup_{k} J_{k}^{-}\left(\bmod \mathcal{H}^{N-1}\right)$.

Proof. Let

$$
\Phi_{j}:=\left\{i: \operatorname{int}\left(J_{j}^{+}\right) \text {is the minimal set int }\left(J_{k}^{+}\right) \text {containing int }\left(J_{i}^{-}\right)\right\} .
$$

By definition the sets $\Phi_{j}$ are pairwise disjoint and the axiom (i) provides for any $i$ a minimal set $\operatorname{int}\left(J_{j}^{+}\right)$containing $\operatorname{int}\left(J_{i}^{-}\right)$, so that $\cup_{j} \Phi_{j}=\mathbb{N}$. We also notice that

$$
Y_{j}=\operatorname{int}\left(J_{j}^{+}\right) \backslash \bigcup_{i \in \Phi_{j}} \operatorname{int}\left(J_{i}^{-}\right) .
$$

because, whenever $\operatorname{int}\left(J_{i}^{-}\right) \subseteq \operatorname{int}\left(J_{j}^{+}\right)$, the maximal set $\operatorname{int}\left(J_{k}^{-}\right) \operatorname{containing} \operatorname{int}\left(J_{i}^{-}\right)$ and contained in $\operatorname{int}\left(J_{j}^{+}\right)$satisfies $k \in \Phi_{j}$, by the axiom (iii).

Finally, the sets $Y_{j}$ are pairwise disjoint because if $\operatorname{int}\left(J_{j}^{+}\right)$and $\operatorname{int}\left(J_{k}^{+}\right)$have a nonempty intersection, then one (say the first) is contained in the other; since there exists $i \in L_{k}$ such that $\operatorname{int}\left(J_{j}^{+}\right) \subseteq \operatorname{int}\left(J_{i}^{-}\right)$we obtain that $Y_{j} \subset \operatorname{int}\left(J_{i}^{-}\right) \subset \mathbb{R}^{\mathrm{N}} \backslash Y_{k}$, a contradiction.

In view of Proposition 3 and (ii'), (iv'), the proof will be complete if we show that

$$
\partial^{\mathrm{M}} Y_{j}=J_{j}^{+} \cup \bigcup_{i \in \Phi_{j}} J_{i}^{-} \quad\left(\bmod \mathcal{H}^{N-1}\right)
$$

for any $j \in \mathbb{N}$. To this aim, we notice that $\mathbb{R}^{\mathrm{N}} \backslash Y_{j}$ is the disjoint union of $\operatorname{ext}\left(J_{j}^{+}\right)$ and $\operatorname{int}\left(J_{i}^{-}\right), i \in \Phi_{j}$; in fact, if $\left|\operatorname{int}\left(J_{i}^{-}\right) \cap \operatorname{int}\left(J_{l}^{-}\right)\right|>0$ for $i, l \in \Phi_{j}, i \neq j$, then one set (say the first) is contained in the other, hence there is a set $\operatorname{int}\left(J_{k}^{+}\right)$ contained $\operatorname{in} \operatorname{int}\left(J_{l}^{-}\right)$and containing $\operatorname{int}\left(J_{i}^{-}\right)$, contradicting the fact that $i \in \Phi_{j}$.

By applying Proposition 3 and (ii') again the identity above follows. 


\section{Topographic function and internal/external boundaries of sets}

The representation of the boundary of a set of finite perimeter by a family of nested Jordan boundaries $J_{i}^{ \pm}$has the advantage of being easily obtained by the family of saturations and holes of the $M$-connected components of $E$, but has the drawback of being not invariant under complementation, as Fig. 2 shows. Another drawback of the $J_{i}^{ \pm}$representation is the absence of a natural order structure on them, despite conditions (i) and (iii) in Theorem 4.
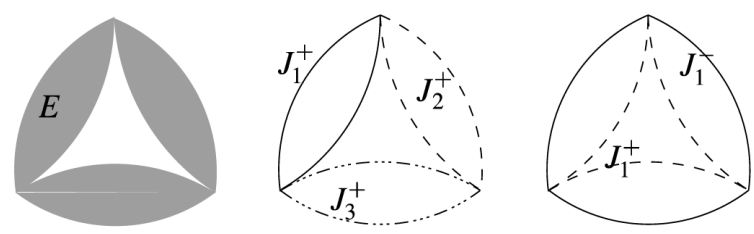

Fig. 2. The set $E$ (in grey), its boundaries $J^{ \pm}$and the boundaries of its complement. The last figure illustrates as well the internal and external boundaries obtained by the topographic function

In this section we prove the existence of a family of nested boundaries which is invariant under complementation; the family is given by $\partial^{\mathrm{M}}\{u \leq k\}$ ( $k$ even for the external boundaries, $k$ odd for the internal ones), where $u: \mathbb{R}^{\mathrm{N}} \rightarrow \mathbb{N}$ is the $\mathrm{BV}_{\text {loc }}$ function characterized by the following theorem. Heuristically, $u(x)$ measures how "deep" is $x$ inside $E$, i.e., it counts how many boundaries must be crossed to reach the exterior of $E$. This is illustrated in Fig. 3 where $E$ is the gray set.

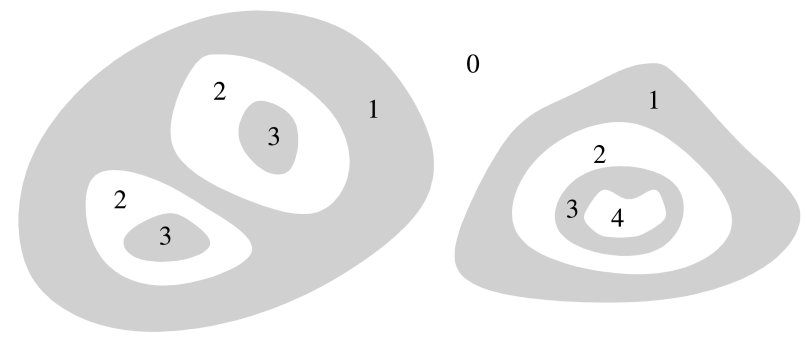

Fig. 3. The topographic function associated with the gray set $E$ counts how many boundaries must be crossed to reach the exterior of $E$

Theorem 6. Let $E \subseteq \mathbb{R}^{\mathrm{N}}$ be a set of finite perimeter. Then there exists a unique map $u \in \mathrm{BV}_{\text {loc }}\left(\mathbb{R}^{\mathrm{N}}, \mathbb{N}\right)$ such that

(i) $u=\chi_{E} \bmod 2$ and all sets $\{u \leq k\}$ are indecomposable;

(ii) $|D u|=\mathcal{H}^{N-1}\left\llcorner\partial^{\mathrm{M}} E\right.$;

(iii) $u=\chi_{E}$ in the $M$-connected component of $E$ or $\mathbb{R}^{\mathrm{N}} \backslash E$ with infinite measure.

Proof. We denote by $\left\{E_{i}\right\}_{i \in I}$ the $M$-connected components of $E$ and by $\left\{F_{j}\right\}_{j \in J}$ the $M$-connected components of $\mathbb{R}^{\mathrm{N}} \backslash E$. Being the statement invariant under 
complementation we can assume that $|E|<\infty$ and denote by $j_{0}$ the index in $J$ such that $\left|F_{j_{0}}\right|=\infty$. Recall that Proposition 3 gives

$$
\partial^{\mathrm{M}}\left(\bigcup_{i \in I_{1}} E_{i}\right)=\bigcup_{i \in I_{1}} \partial^{\mathrm{M}} E_{i}, \quad \partial^{\mathrm{M}}\left(\bigcup_{j \in J_{1}} F_{j}\right)=\bigcup_{j \in J_{1}} \partial^{\mathrm{M}} F_{j} \quad\left(\bmod \mathcal{H}^{N-1}\right)
$$

whenever $I_{1} \subseteq I, J_{1} \subseteq J$.

(Existence) We define recursively sets $U_{k} \subseteq \mathbb{R}^{\mathrm{N}}$ and subsets $\Phi_{k} \subset I$, for $k$ odd, and $\Phi_{k} \subset J$ for $k$ even as follows: first we set $U_{0}=F_{j_{0}}$ and $\Phi_{0}=\left\{j_{0}\right\}$ and then, assuming that all sets $U_{l}$ and $\Phi_{l}$ have been defined for $l<k$, we define:

$$
\Phi_{k}:=\left\{i \in I: \mathcal{H}^{N-1}\left(\partial^{\mathrm{M}} U_{k-1} \cap \partial^{\mathrm{M}} E_{i}\right)>0\right\}, \quad U_{k}:=U_{k-1} \cup \bigcup_{i \in \Phi_{k}} E_{i} \text { if } k \text { is odd }
$$

and

$\Phi_{k}:=\left\{j \in J: \mathcal{H}^{N-1}\left(\partial^{\mathrm{M}} U_{k-1} \cap \partial^{\mathrm{M}} F_{j}\right)>0\right\}, \quad U_{k}:=U_{k-1} \cup \bigcup_{j \in \Phi_{k}} F_{j}$ if $k$ is even.

Let us prove by induction that all sets $U_{k}$ are indecomposable. This property is clearly satisfied for $k=0$, so let us assume it true for $k-1 \geq 0$ and let us prove it for $k$. Assuming, to fix the ideas, that $k$ is odd, for any finite set $R \subset \Phi_{k}$ and any $i \in \Phi_{k} \backslash R$ we have

$$
\partial^{\mathrm{M}} E_{i} \cap \partial^{\mathrm{M}}\left(U_{k-1} \cup \bigcup_{i \in R} E_{i}\right) \supseteq \partial^{\mathrm{M}} E_{i} \cap \partial^{\mathrm{M}} U_{k-1} \neq \emptyset \quad\left(\bmod \mathcal{H}^{N-1}\right)
$$

because $\partial^{\mathrm{M}} E_{i}$ are pairwise disjoint $\left(\bmod \mathcal{H}^{N-1}\right)$. Hence, by applying inductively Proposition 5(i), we obtain that $U_{k-1} \cup \bigcup_{i \in R} E_{i}$ is indecomposable for any finite set $R \subseteq \Phi_{k}$. By Proposition 5(ii) we obtain that $U_{k}$ is indecomposable. An analogous argument also proves that

$$
\partial^{\mathrm{M}} U_{k} \subseteq \partial^{\mathrm{M}} E \quad\left(\bmod \mathcal{H}^{N-1}\right) \quad \forall k \in \mathbb{N} .
$$

Denoting by $I^{\prime}$ (respectively $J^{\prime}$ ) the subset of $I$ (resp. of $J$ ) obtained by taking the union of all sets $\Phi_{2 k+1}$ (resp. $\Phi_{2 k}$ ), let us prove the following two upper and lower bounds on $\partial^{\mathrm{M}} U_{k}$, which both will be useful in the following:

$$
\begin{aligned}
\partial^{\mathrm{M}} U_{2 k} \cup \partial^{\mathrm{M}} U_{2 k+1} & \subseteq \bigcup_{i \in \Phi_{2 k+1}} \partial^{\mathrm{M}} E_{i}, \quad \partial^{\mathrm{M}} U_{2 k-1} \cup \partial^{\mathrm{M}} U_{2 k} \\
& \subseteq \bigcup_{j \in \Phi_{2 k}} \partial^{\mathrm{M}} F_{j}\left(\bmod \mathcal{H}^{N-1}\right)
\end{aligned}
$$

and

$$
\begin{aligned}
\bigcup_{i \in \Phi_{2 k+1}} \partial^{\mathrm{M}} E_{i} \backslash \partial^{\mathrm{M}} U_{2 k+1} & \subseteq \bigcup_{j \in J^{\prime}} \partial^{\mathrm{M}} F_{j}, \bigcup_{j \in \Phi_{2 k}} \partial^{\mathrm{M}} F_{j} \backslash \partial^{\mathrm{M}} U_{2 k} \\
& \subseteq \bigcup_{i \in I^{\prime}} \partial^{\mathrm{M}} E_{i}\left(\bmod \mathcal{H}^{N-1}\right) .
\end{aligned}
$$


The first inclusion in (23) follows by

$$
\partial^{\mathrm{M}} U_{2 k+1} \subset \partial^{\mathrm{M}} U_{2 k} \cup \partial^{\mathrm{M}}\left(\bigcup_{i \in \Phi_{2 k+1}} E_{i}\right)
$$

using (22) and the definition of $\Phi_{2 k+1}$; the second inclusion can be proved in a similar way. The first inclusion in (24) follows by the second one in (23), noticing that

$$
\partial^{\mathrm{M}}\left(\bigcup_{i \in \Phi_{2 k+1}} E_{i}\right) \subset \partial^{\mathrm{M}} U_{2 k+1} \cup \partial^{\mathrm{M}} U_{2 k}
$$

The proof of the second inclusions in (24) is analogous.

Now we prove that $\Omega=\cup_{k} U_{k}$ is $\mathbb{R}^{\mathrm{N}}\left(\bmod \mathcal{H}^{N}\right)\left(\right.$ hence $I^{\prime}=I$ and $\left.J^{\prime}=J\right)$. To this aim, we argue by contradiction: since

$$
\partial^{\mathrm{M}} \Omega \subset \partial^{\mathrm{M}}\left(\bigcup_{i \in I^{\prime}} E_{i}\right) \cup \partial^{\mathrm{M}}\left(\bigcup_{j \in J^{\prime}} F_{j}\right)=\bigcup_{i \in I^{\prime}} \partial^{\mathrm{M}} E_{i} \cup \bigcup_{j \in J^{\prime}} \partial^{\mathrm{M}} F_{j} \quad\left(\bmod \mathcal{H}^{N-1}\right)
$$

and an analogous property holds for $\mathbb{R}^{\mathrm{N}} \backslash \Omega$ and $I \backslash I^{\prime}, J \backslash J^{\prime}$, taking into account that $\forall i \in I \backslash I^{\prime}, \forall j \in J \backslash J^{\prime}, \partial^{\mathrm{M}} E_{i}$ and $\partial^{\mathrm{M}} F_{j}$ are pairwise disjoint $\left(\bmod \mathcal{H}^{N-1}\right)$, assuming that $P(\Omega)>0$ we can find either $i \in I \backslash I^{\prime}$ and $j \in J^{\prime}$ or $i \in I^{\prime}$ and $j \in J \backslash J^{\prime}$ such that $\partial^{\mathrm{M}} E_{i} \cap \partial^{\mathrm{M}} F_{j} \neq \varnothing\left(\bmod \mathcal{H}^{N-1}\right)$. Assume, to fix the ideas, that $i \in I \backslash I^{\prime}$ and $j \in J^{\prime}$ and let $k$ such that $j \in \Phi_{2 k}$. Then, by (23) and (24) we obtain that

$$
\partial^{\mathrm{M}} U_{2 k} \cap \partial^{\mathrm{M}} E_{i} \neq \emptyset \quad\left(\bmod \mathcal{H}^{N-1}\right) .
$$

This proves that $i \in \Phi_{2 k+1} \subseteq I^{\prime}$ and gives a contradiction.

Finally, we define $u$ equal to $k$ on $U_{k} \backslash U_{k-1}$ (with $U_{-1}=\emptyset$ ). By construction $\{u \leq k\}=U_{k}$ is indecomposable and $u=\chi_{E} \bmod 2$. Let us prove that condition (ii) holds; to this aim, we first prove that all sets $\Phi_{2 k+1}$ are pairwise disjoint. Assume by contradiction that $i \in \Phi_{2 l+1} \cap \Phi_{2 k+1}$ with $l<k$; then $E_{i} \subseteq U_{2 l+1} \subseteq U_{2 k}$ and the inclusions

$$
\partial^{\mathrm{M}} U_{2 k} \cap \partial^{\mathrm{M}} E_{i} \neq \emptyset, \quad \partial^{\mathrm{M}} U_{2 k} \subseteq \bigcup_{j \in \Phi_{2 k}} \partial^{\mathrm{M}} F_{j} \quad\left(\bmod \mathcal{H}^{N-1}\right)
$$

imply the existence of $j \in \Phi_{2 k}$ and $x \in\left(E_{i}\right)^{1 / 2} \cap \partial^{\mathrm{M}} U_{2 k} \cap\left(F_{j}\right)^{1 / 2}$. Since $U_{2 k}$ contains both $E_{i}$ and $F_{j}$ we obtain that $x \in \stackrel{\circ}{U}_{2 k}^{\mathrm{M}}$ and this is a contradiction.

Now, since the sets $\Phi_{2 k+1}$ are pairwise disjoint, the first inclusion in (23) implies that $\mathcal{H}^{N-1}\left(\partial^{\mathrm{M}} U_{k} \cap \partial^{\mathrm{M}} U_{l}\right)=0$ whenever $k \neq l$. Moreover, (22) and (25) imply that $\cup_{k} \partial^{\mathrm{M}} U_{k}=\partial^{\mathrm{M}} E\left(\bmod \mathcal{H}^{N-1}\right)$. Since $u=\sum_{k} \chi_{\mathbb{R}^{\mathrm{N}} \backslash U_{k}}$ we obtain

$$
|D u|=\left|\sum_{k} D \chi_{\mathbb{R}^{\mathrm{N}} \backslash U_{k}}\right|=\left|\sum_{k} D \chi_{U_{k}}\right|=\mathcal{H}^{N-1}\left\llcorner\cup_{k} \partial^{\mathrm{M}} U_{k}=\mathcal{H}^{N-1}\left\llcorner\partial^{\mathrm{M}} E .\right.\right.
$$


(Uniqueness) Let $v$ be satisfying (i), (ii), (iii) and let us prove that $v$ coincides with the function $u$ constructed above. First of all, notice that condition (ii) implies that $v$ is (equivalent to) a constant in any $M$-connected component of $E$ or $\mathbb{R}^{\mathrm{N}} \backslash E$, by the constancy theorem (see Remark 2$)$. Moreover, $\partial^{\mathrm{M}} E$ coincides $\left(\bmod \mathcal{H}^{N-1}\right)$ with the jump set of $v$ and $\left|v^{+}-v^{-}\right|$(i.e., the width of the jump) is $1 \mathcal{H}^{N-1}$-a.e. in $\mathbb{R}^{\mathrm{N}}$ (see Sect. 3).

By condition (iii) the two functions are both 0 on $F_{j_{0}}$. Let $i \in \Phi_{1}$; since

$$
\partial^{\mathrm{M}} E \supseteq \partial^{\mathrm{M}} E_{i} \cap \partial^{\mathrm{M}} F_{j_{0}} \neq \varnothing \quad\left(\bmod \mathcal{H}^{N-1}\right)
$$

we obtain that $v$ must be equal to 1 on $E_{i}$. Being $i$ arbitrary, this proves that $v$ coincides with $u$ on $U_{1}$. Consider now $j \in \Phi_{2}$; the same argument exploited before proves that either $v$ is a.e. equal to 2 or $v$ is a.e. equal to 0 in $F_{j}$. The second possibility can be excluded noticing that in this case the set $\{v \leq 0\}$ would be decomposable: indeed, by (23) we get

$$
\partial^{\mathrm{M}} U_{0} \subseteq \bigcup_{i \in \Phi_{1}} \partial^{\mathrm{M}} E_{i} \cap \partial^{\mathrm{M}} U_{0} \subseteq \partial^{\mathrm{M}}\{v \leq 0\} \quad\left(\bmod \mathcal{H}^{N-1}\right)
$$

and, passing to the complementary sets $\partial^{\mathrm{M}}\left(\{v \leq 0\} \backslash U_{0}\right) \subset \partial^{\mathrm{M}}\{v \leq 0\}\left(\bmod \mathcal{H}^{N-1}\right)$, so that Proposition 3(iv) gives

$$
P(\{v \leq 0\})=P\left(U_{0}\right)+P\left(\{v \leq 0\} \backslash U_{0}\right) .
$$

Continuing by induction in this way and using the inclusions $\left(\bmod \mathcal{H}^{N-1}\right)$ (the first for $k$ even, the second for $k$ odd, coming from (23) and the inductive assumption)

$$
\begin{aligned}
& \partial^{\mathrm{M}} U_{k-2} \subseteq \bigcup_{i \in \Phi_{k-1}} \partial^{\mathrm{M}} E_{i} \cap \partial^{\mathrm{M}} U_{k-2} \subseteq \partial^{\mathrm{M}}\{v \leq k-2\}, \\
& \partial^{\mathrm{M}} U_{k-2} \subseteq \bigcup_{j \in \Phi_{k-1}} \partial^{\mathrm{M}} F_{j} \cap \partial^{\mathrm{M}} U_{k-2} \subseteq \partial^{\mathrm{M}}\{v \leq k-2\}
\end{aligned}
$$

we obtain that $v$ coincides with $u$ on $U_{k}$. Since $k$ is arbitrary, this proves that $v=u$.

Definition 6 (Topographic function). We call the function given by the previous theorem the topographic function of $E$, and denote it by $u_{E}$. We also call the sets

$$
\partial^{\mathrm{M}}\left\{u_{E} \leq 2 k\right\}, \quad \partial^{\mathrm{M}}\left\{u_{E} \leq 2 k+1\right\} \quad k \in \mathbb{N}
$$

respectively the external and the internal boundaries of $E$.

Notice that

$$
u_{E}+1=u_{\mathbb{R}^{\mathrm{N}} \backslash E} \quad \text { whenever }|E|<\infty
$$

because it is easy to check that $u_{E}+1$ fulfils (i), (ii), (iii) with $\mathbb{R}^{\mathrm{N}} \backslash E$ in place of $E$. As a consequence, complementation maps internal (external) boundaries into external (internal) boundaries. Passing to the complementary sets, the identity above can also be written as $u_{E}=u_{\mathbb{R}^{\mathrm{N}} \backslash E}+1$ whenever $|E|=\infty$. In particular, in this case the topographic function achieves its minimum, equal to 1 , on the component of $E$ with infinite measure (if $|E|<\infty$ the minimum is 0 , by condition (iii)). 


\section{Indecomposability and Jordan curves in the plane}

The aim of this section is a closer characterization of the $M$-connected components and of the essential boundary for plane sets of finite perimeter. In particular we prove that $\partial^{\mathrm{M}} E$ can be represented $\left(\bmod \mathcal{H}^{1}\right)$ as a disjoint union of rectifiable Jordan curves; this result has been proved first for simple sets by W.H. Fleming in [25] (see also [26]) and later extended to the general case by H. Federer (see [24], 4.2.25). We also prove that membership to the same $M$-connected component can be characterized in terms of existence of arcs joining the points and not touching (in a suitable sense) the boundary.

We say that $\Gamma \subseteq \mathbb{R}^{2}$ is a Jordan curve if $\Gamma=\gamma([a, b])$ for some $a, b \in \mathbb{R}$ (with $a<b$ ) and some continuous map $\gamma$, one-to-one on $[a, b)$ and such that $\gamma(a)=\gamma(b)$. In a more geometric language, $\Gamma$ can be viewed as the image of a continuous and one-to-one map defined on the unit circle $\mathbf{S}^{1}$. According to the celebrated Jordan curve theorem (see for instance [35]), any Jordan curve $\Gamma$ splits $\mathbb{R}^{2} \backslash \Gamma$ in exactly two connected components, a bounded one and an unbounded one, whose common boundary is $\Gamma$. As for Jordan boundaries, these components will be respectively denoted by $\operatorname{int}(\Gamma)$ and $\operatorname{ext}(\Gamma)$. We will also use the signed distance function $\operatorname{sdist}(x, \Gamma)$, defined by

$$
\operatorname{sdist}(x, \Gamma):=\left\{\begin{array}{r}
-\operatorname{dist}(x, \Gamma) \text { if } x \in \operatorname{int}(\Gamma) \cup \Gamma ; \\
\operatorname{dist}(x, \Gamma) \text { if } x \in \operatorname{ext}(\Gamma) \cup \Gamma .
\end{array}\right.
$$

In our context, we are more interested in Lipschitz parameterizations rather than continuous ones; the main tool for providing them is the following well known lemma.

Lemma 3 (Connectedness by arcs). Let $C \subset \mathbb{R}^{\mathrm{N}}$ be a compact connected set with $\mathcal{H}^{1}(C)<\infty$. Then for any pair of distinct points $x, y \in C$ there exists a Lipschitz one-to-one map $\gamma:[0,1] \rightarrow C$ such that $\gamma(0)=x$ and $\gamma(1)=y$.

Proof. The existence of a Lipschitz map (not necessarily one-to-one) joining $x$ to $y$ is proved in [23]. In order to obtain a one-to-one map it suffices to look for solutions of the problem

$$
\min \left\{\int_{a}^{b}\left|\gamma^{\prime}(t)\right| d t:[a, b] \subseteq \mathbb{R}, \quad \gamma \in \operatorname{Lip}([a, b], C), \gamma(a)=x, \gamma(b)=y\right\} .
$$

Existence of minimizers is a straightforward consequence of Ascoli-Arzelá theorem and of a classical reparameterization argument. Clearly any minimizer $\gamma_{0}$, when parameterized by arc length, is one-to-one. A final reparameterization gives $\gamma:[0,1] \rightarrow C$.

A first consequence of Lemma 3 is the fact that any Jordan curve $\Gamma$ with $\mathcal{H}^{1}(\Gamma)<\infty$ admits a Lipschitz reparameterization. In fact, let $x, y \in \Gamma$ with $x \neq y$, let $\gamma:[0,1] \rightarrow \Gamma$ be given by Lemma 3 and let $\tilde{\Gamma}=\Gamma \backslash \gamma((0,1))$. Since $\tilde{\Gamma}$ is homeomorphic to a closed segment, Lemma 3 again gives a Lipschitz 
homeomorphism $\tilde{\gamma}:[1,2] \rightarrow \tilde{\Gamma}$ with $\tilde{\gamma}(1)=y$ and $\tilde{\gamma}(2)=x$. Joining $\gamma$ and $\tilde{\gamma}$ we obtain the desired Lipschitz parameterization of $\Gamma$. In the following we call rectifiable the Jordan curves such that $\mathcal{H}^{1}(\Gamma)<\infty$. More generally, any $\Gamma=$ $\gamma([a, b])$ with $\gamma$ Lipschitz function in $[a, b]$ will be called rectifiable curve.

In the following lemma we point out some mild regularity properties of rectifiable Jordan curves which will be used in the following.

Lemma 4. Let $\Gamma \subset \mathbb{R}^{2}$ be a rectifiable Jordan curve. Then

$$
\begin{gathered}
\mathcal{H}^{1}(\Gamma \cap B(x, r / 2)) \geq r \quad \forall x \in \Gamma, r \in(0, \operatorname{diam}(\Gamma)), \\
\mathcal{H}^{1}(\Gamma)=P(\operatorname{int}(\Gamma))=P(\operatorname{ext}(\Gamma))
\end{gathered}
$$

and

$$
\liminf _{r \rightarrow 0^{ \pm}} \mathcal{H}^{1}\left(\left\{x \in \mathbb{R}^{2}: \operatorname{sdist}(x, \Gamma)=r\right\}\right)=\mathcal{H}^{1}(\Gamma) .
$$

Proof. The first property can be easily proved by a projection argument, see for instance Lemma 3.4 of [23], taking into account that $\Gamma$ intersects at least twice $\partial B(x, r / 2)$.

In order to prove the second one, let us represent $\Gamma$ as $\gamma([0,1])$ with $\gamma:[0,1] \rightarrow$ $\mathbb{R}^{2}$ satisfying $\left|\gamma^{\prime}(t)\right|=1$ for a.e. $t \in[0,1]$ and let $x_{0} \in \Gamma$ such that

$$
\limsup _{\rho \rightarrow 0^{+}} \frac{\mathcal{H}^{1}\left(\Gamma \cap B_{\rho}\left(x_{0}\right)\right)}{2 \rho} \leq 1
$$

and, for $t_{0}=\gamma^{-1}\left(x_{0}\right), \gamma$ is differentiable at $t_{0}$ and $\left|\gamma^{\prime}\left(t_{0}\right)\right|=1$; notice that $\mathcal{H}^{1}$-a.e. $x_{0} \in \Gamma$ has these properties. The coarea formula (see 3.2.3 of [24]) gives

$$
\int_{0}^{\rho} \operatorname{card}\left(\Gamma \cap \partial B_{r}\left(x_{0}\right)\right) d r \leq \mathcal{H}^{1}\left(\Gamma \cap B_{\rho}\left(x_{0}\right)\right) \quad \forall \rho>0
$$

and hence we can find arbitrarily small $r>0$ such that $\Gamma \cap \partial B_{r}\left(x_{0}\right)$ contains two points $x_{r}, y_{r}$; by the differentiability of $\gamma$ at $t_{0}$ we have also that $\left|x_{r}-y_{r}\right| / 2 r$ tends to 1 as $r \rightarrow 0^{+}$. Denoting by $J_{r}^{ \pm} \subset \partial B_{r}\left(x_{0}\right)$ the circular arcs joining $x_{r}$ and $y_{r}$, we obtain that $J_{r}^{ \pm} \cup\left(\Gamma \cap B_{r}\left(x_{0}\right)\right)$ are Jordan curves, whose interiors are the connected components of $B_{r}\left(x_{0}\right) \backslash \Gamma$. It follows that one of these components is contained in $\operatorname{int}(\Gamma)$ and the other one in $\operatorname{ext}(\Gamma)$, and since the angle between $x_{r}$ and $y_{r}$ tends to $\pi$ as $r \rightarrow 0^{+}$we obtain that $x_{0}$ is a point of density $1 / 2$ for $\operatorname{int}(\Gamma)$ and $\operatorname{ext}(\Gamma)$. This proves that

$\mathcal{H}^{1}(\Gamma) \leq \mathcal{H}^{1}\left(\partial^{\mathrm{M}} \operatorname{int}(\Gamma)\right)=P(\operatorname{int}(\Gamma)), \quad \mathcal{H}^{1}(\Gamma) \leq \mathcal{H}^{1}\left(\partial^{\mathrm{M}} \operatorname{ext}(\Gamma)\right)=P(\operatorname{ext}(\Gamma))$

The opposite inequalities follow by the inclusions $\partial \operatorname{int}(\Gamma) \subset \Gamma, \partial \operatorname{ext}(\Gamma) \subset \Gamma$.

In order to prove the third property we set $\phi(x)=\operatorname{sdist}(x, \Gamma)$ and recall (see for instance [24], 3.2.11, 3.2.34) that $|\nabla \phi|=1$ a.e. in $\mathbb{R}^{2}$, so that the coarea formula gives

$$
\left|\phi^{-1}(-r, r)\right|=\int_{\phi^{-1}(-r, r)}|\nabla \phi| d x=\int_{-r}^{r} \mathcal{H}^{1}\left(\left\{x \in \mathbb{R}^{2}: \phi(x)=t\right\}\right) d t \quad \forall r>0 .
$$


On the other hand, it can be proved (see 3.2.39 of [24] or Theorem 2.106 of [5]) that $\left|\phi^{-1}(-r, r)\right| /(2 r)$ tends to $\mathcal{H}^{1}(\Gamma)$ as $r \rightarrow 0^{+}$. Hence we can find an infinitesimal sequence of positive numbers $r_{i}$ such that

$$
\limsup _{i \rightarrow \infty} \mathcal{H}^{1}\left(\left\{x \in \mathbb{R}^{2}:|\phi(x)|=r_{i}\right\}\right) \leq 2 \mathcal{H}^{1}(\Gamma) .
$$

On the other hand, the lower semicontinuity of perimeter and (28) give

$$
\left.\liminf _{i \rightarrow \infty} \mathcal{H}^{1}\left(\left\{x \in \mathbb{R}^{2}: \phi(x)=r_{i}\right\}\right) \geq \liminf _{i \rightarrow \infty} P\left(\left\{\phi<r_{i}\right\}\right) \geq P(\phi<0\}\right)=\mathcal{H}^{1}(\Gamma)
$$

and, analogously, $\liminf \operatorname{in}_{i} \mathcal{H}^{1}\left(\left\{x \in \mathbb{R}^{2}: \phi(x)=-r_{i}\right\}\right) \geq \mathcal{H}^{1}(\Gamma)$. These inequalities imply that both $\mathcal{H}^{1}\left(\left\{\phi=r_{i}\right\}\right)$ and $\mathcal{H}^{1}\left(\left\{\phi=-r_{i}\right\}\right)$ converge to $\mathcal{H}^{1}(\Gamma)$ as $i \rightarrow \infty$.

In order to represent the essential boundary of a simple set by a rectifiable Jordan curve we need the following lemma.

Lemma 5. Let $\gamma:[0, L] \rightarrow \mathbb{R}^{2}$ be a Lipschitz map, let $C=\gamma([0, L])$ and assume that $\gamma(0)=\gamma(L)$ and $\int_{0}^{L}\left|\gamma^{\prime}\right| d t=\mathcal{H}^{1}(C)>0$. Then $C$ contains a rectifiable Jordan curve $\Gamma$.

Proof. After reparameterization we can assume with no loss of generality that $L=\mathcal{H}^{1}(C)$ and $\left|\gamma^{\prime}\right|=1$ a.e. in $[0, L]$. By the area formula (see for instance [22])

$\left|\gamma^{-1}(A)\right|=\int_{\gamma^{-1}(A)}\left|\gamma^{\prime}(t)\right| d t=\int_{A} \operatorname{card}\left(\gamma^{-1}(x)\right) d \mathcal{H}^{1}(x) \quad \forall A \subseteq C, A$ Borel with $A=C$ we obtain

$$
\int_{C}\left(\operatorname{card}\left(\gamma^{-1}(x)\right)-1\right) d \mathcal{H}^{1}(x)=\int_{0}^{1}\left|\gamma^{\prime}(t)\right| d t-\mathcal{H}^{1}(C)=0
$$

hence the set $B=\left\{x: \operatorname{card}\left(\gamma^{-1}(x)\right)>1\right\}$ is $\mathcal{H}^{1}$-negligible, and so is (again by the area formula with $A=B$ ) the set $S=\gamma^{-1}(B)$.

We now claim that $\bar{S}$ is still Lebesgue negligible. In fact, let $\left(t_{h}\right) \subseteq S$ be converging to $t$ and let $s_{h} \neq t_{h}$ such that $\gamma\left(t_{h}\right)=\gamma\left(s_{h}\right)$; assuming with no loss of generality that $s_{h}$ converge to $s$, if $s \neq t$ we conclude that $t \in S$, otherwise if $s=t$ we obtain that either $\gamma$ is not differentiable at $t$ or $\gamma^{\prime}(t)=0$. This proves that $\bar{S}$ is Lebesgue negligible.

Take now a connected component $(a, b)$ of $(0,1) \backslash \bar{S}$ and consider the simple arc $C^{\prime}=\gamma((a, b))$. Since $C \backslash C^{\prime}$ is connected (being $\gamma$ a closed curve), by Lemma 3 we can connect $\gamma(b)$ to $\gamma(a)$ by a simple path $\eta:[b, c] \rightarrow C \backslash C^{\prime}$. If $\gamma(a)=\gamma(b)$, then $C^{\prime}$ is a Jordan curve. If $\gamma(a) \neq \gamma(b)$, then a Jordan curve contained in $C$ can be obtained joining the paths $\left.\gamma\right|_{[a, b]}$ and $\left.\eta\right|_{[b, c]}$.

Theorem 7 (Boundary of simple plane sets). Let $E \subset \mathbb{R}^{2}$ be a simple set with $|E| \in(0, \infty)$. Then $E$ is (essentially) bounded and $\partial^{\mathrm{M}} E$ is equivalent $\left(\bmod \mathcal{H}^{1}\right)$ to a rectifiable Jordan curve. Conversely, $\operatorname{int}(\Gamma)$ is a simple set for any rectifiable Jordan curve $\Gamma$. 
Proof. By a rescaling argument we also assume that $P(E)<1$. Let $\left(E_{h}\right)$ be a sequence of bounded open sets with smooth boundary locally converging in measure to $E$ and such that $P\left(E_{h}\right) \rightarrow P(E)$ as $h \rightarrow \infty$. Since $\partial E_{h}$ is smooth and compact, we can represent it by a disjoint union of Jordan curves $\Gamma_{i, h}$, for $1 \leq i \leq$ $N(h)$, whose length decreases as $i$ increases; we parameterize $\Gamma_{i, h}=\gamma_{i, h}([0,1])$ for some 1-Lipschitz maps $\gamma_{i, h}$, one-to-one on $[0,1)$, and notice that

$$
\sum_{i=1}^{N(h)} \int_{0}^{1}\left|\gamma_{i, h}^{\prime}(t)\right| d t=\sum_{i=1}^{N(h)} \mathcal{H}^{1}\left(\Gamma_{i, h}\right)=P\left(E_{h}\right)<1
$$

for $h$ large enough. In the following we assume, to fix the ideas, that $N(h) \rightarrow \infty$ as $h \rightarrow \infty$, the proof being much simpler if $N(h) \leq C$ for infinitely many $h$. We assume, possibly extracting a subsequence, that for any $i \in \mathbb{N}$ either $\gamma_{i, h}$ uniformly converge in $[0,1]$ to $\gamma_{i}$ or $\max \left|\gamma_{i, h}\right| \rightarrow \infty$. In the latter case we set $\gamma_{i} \equiv 0$. Setting $\Gamma_{i}=\gamma_{i}([0,1])$ and $\Gamma_{\infty}=\cup_{i} \Gamma_{i}$, we will prove that there exists $i$ such that $\Gamma_{i}$ is a Jordan curve and $\Gamma_{j}$ are points for any $j \neq i$.

Step 1. We claim that $\partial^{\mathrm{M}} E \subset \Gamma_{\infty}\left(\bmod \mathcal{H}^{1}\right)$. Given an integer $p \geq 1$, we denote by $E_{h}^{p}$ the sets obtained from $E_{h}$ by removing from it the connected components with area smaller than $1 / p$ and adding to it all holes with area smaller than $1 / p$. By the isoperimetric inequality, the perimeter of any connected component of $E_{h}^{p}$ is at least $\sqrt{4 \pi / p}$, hence $\partial E_{h}^{p}$ is contained in the first $M_{p}=[\sqrt{p /(4 \pi)}]+1$ curves $\Gamma_{i, h}$. Moreover, we have

$$
\left|E_{h}^{p} \Delta E_{h}\right| \leq \sum_{j \in J}\left|Y_{j}\right| \leq \frac{1}{\sqrt{p}} \sum_{j \in J}\left|Y_{j}\right|^{1 / 2} \leq \frac{1}{\sqrt{4 \pi p}} \sum_{j \in J} P\left(Y_{j}\right) \leq \frac{1}{\sqrt{4 \pi p}}
$$

where $\left\{Y_{j}\right\}_{j \in J}$ are the components added or removed. We assume, without loss of generality, that $E_{h}^{p}$ locally converge in measure in $\mathbb{R}^{\mathrm{N}}$ to suitable sets $E^{p}$ as $h \rightarrow \infty$ such that $\left|E^{p} \Delta E\right| \leq 1 / \sqrt{4 \pi p}$. Since

$$
\partial E_{h}^{p} \subset \bigcup_{i=1}^{M_{p}} \Gamma_{i, h}
$$

and since $D \chi_{E_{h}^{p}}$ weakly converge as measures to $D \chi_{E^{p}}$, by the definition of $\Gamma_{i}$ we easily obtain that

$$
\left|D \chi_{E^{p}}\right| \leq \mathcal{H}^{1}\left\llcorner\bigcup_{i=1}^{M_{p}} \Gamma_{i}\right.
$$

because any closed ball disjoint from the set in the right side does not intersect $\Gamma_{i, h}$, $1 \leq i \leq M_{p}$, for $h$ large enough. Hence, $\left|D \chi_{E^{p}}\right| \leq \mathcal{H}^{1}\left\llcorner\Gamma_{\infty}\right.$ for any $p$. Letting $p \rightarrow \infty$ and using the weak convergence of $E_{p}$ to $E$ we get $\left|D \chi_{E}\right| \leq \mathcal{H}^{1}\left\llcorner\Gamma_{\infty}\right.$. The claim follows by evaluating both measures at $\partial^{\mathrm{M}} E \backslash \Gamma_{\infty}$. 
Step 2. Passing to the limit as $h \rightarrow \infty$ in (30) we get

$$
\sum_{i=1}^{\infty} \mathcal{H}^{1}\left(\Gamma_{i}\right) \leq \sum_{i=1}^{\infty} \int_{0}^{1}\left|\gamma_{i}^{\prime}\right| d t \leq P(E)=\mathcal{H}^{1}\left(\partial^{\mathrm{M}} E\right)
$$

On the other hand, Step 1 gives

$$
\mathcal{H}^{1}\left(\partial^{\mathrm{M}} E\right) \leq \mathcal{H}^{1}\left(\cup_{i} \Gamma_{i}\right) \leq \sum_{i=1}^{\infty} \mathcal{H}^{1}\left(\Gamma_{i}\right)
$$

Hence, we conclude that $\int_{0}^{1}\left|\gamma_{i}^{\prime}(t)\right| d t=\mathcal{H}^{1}\left(\Gamma_{i}\right)$ for any $i \geq 1$ and $\mathcal{H}^{1}\left(\Gamma_{i} \cap \Gamma_{j}\right)=0$ whenever $i \neq j$.

Step 3. Let $i \geq 1$ such that $\mathcal{H}^{1}\left(\Gamma_{i}\right)>0$ and let $\Gamma \subset \Gamma_{i}$ be a Jordan curve given by Lemma 5. Then, $F=$ int $\Gamma$ satisfies

$$
\partial^{\mathrm{M}} F \subset \Gamma \subset \Gamma_{i} \subset \partial^{\mathrm{M}} E \quad\left(\bmod \mathcal{H}^{1}\right)
$$

so that, being $E$ simple, we conclude from Proposition 7 that $E=F$ and $\partial^{\mathrm{M}} E=$ $\Gamma=\Gamma_{i}\left(\bmod \mathcal{H}^{1}\right)$. This also proves that $\mathcal{H}^{1}\left(\Gamma_{j}\right)=0$ for any $j \neq i$. Since diam $\Gamma \leq \mathcal{H}^{1}(\Gamma)$ for any rectifiable Jordan curve $\Gamma$ we obtain that $E$ is bounded.

Finally, the fact that any rectifiable Jordan curve induces a simple set follows by Proposition 2 and by the Jordan curve theorem.

By Theorem 4, since Jordan boundaries essentially coincide with rectifiable Jordan curves, we obtain the following decomposition result for the boundary of a set of finite perimeter in the plane. As in Theorem 4 we allow the Jordan curves to be also $J_{\infty}$ and $J_{o}$ to simplify the statement and to allow sets $E$ with infinite measure.

Corollary 1. Let $E$ be a subset of $\mathbb{R}^{2}$ of finite perimeter. Then, there is a unique decomposition of $\partial^{\mathrm{M}} E$ into rectifiable Jordan curves $\left\{C_{i}^{+}, C_{k}^{-}: i, k \in \mathbb{N}\right\} \subset \mathcal{S}$, such that

(i) Given $\operatorname{int}\left(C_{i}^{+}\right), \operatorname{int}\left(C_{k}^{+}\right), i \neq k$, they are either disjoint or one is contained in the other; given $\operatorname{int}\left(C_{i}^{-}\right), \operatorname{int}\left(C_{k}^{-}\right), i \neq k$, they are either disjoint or one is contained in the other. Each $\operatorname{int}\left(C_{i}^{-}\right)$is contained in one of the $\operatorname{int}\left(C_{k}^{+}\right)$.

(ii) $P(E)=\sum_{i} \mathcal{H}^{1}\left(C_{i}^{+}\right)+\sum_{k} \mathcal{H}^{1}\left(C_{k}^{-}\right)$.

(iii) If $\operatorname{int}\left(C_{i}^{+}\right) \subseteq \operatorname{int}\left(C_{j}^{+}\right), i \neq j$, then there is some rectifiable Jordan curve $C_{k}^{-}$ such that $\operatorname{int}\left(C_{i}^{+}\right) \subseteq \operatorname{int}\left(C_{k}^{-}\right) \subseteq \operatorname{int}\left(C_{j}^{+}\right)$. Similarly, if $\operatorname{int}\left(C_{i}^{-}\right) \subseteq \operatorname{int}\left(C_{j}^{-}\right)$, $i \neq j$, then there is some rectifiable Jordan curve $C_{k}^{+}$such that $\operatorname{int}\left(C_{i}^{-}\right) \subseteq$ $\operatorname{int}\left(C_{k}^{+}\right) \subseteq \operatorname{int}\left(C_{j}^{-}\right)$.

(iv) Setting $L_{j}=\left\{i: \operatorname{int}\left(C_{i}^{-}\right) \subseteq \operatorname{int}\left(C_{j}^{+}\right)\right\}$, the sets $Y_{j}=\operatorname{int}\left(C_{j}^{+}\right) \backslash \cup_{i \in L_{j}} \operatorname{int}\left(C_{i}^{-}\right)$ are pairwise disjoint, indecomposable and $E=\cup_{j} Y_{j}$. 
In the remaining part of this section we want to characterize the $M$-connected components (or, better, suitable representatives in the equivalence class $\left(\bmod \mathcal{H}^{2}\right)$ ), by the classical topological property of connectedness by arcs.

To this aim, we need another definition of boundary which, more than $\partial^{\mathrm{M}}$, is suitable for the analysis of connected components. For any set $E$ with finite perimeter in $\mathbb{R}^{\mathrm{N}}$ we define

$$
\partial^{S} E:=\left\{x \in \mathbb{R}^{\mathrm{N}}: \limsup _{r \rightarrow 0^{+}} \frac{\mathcal{H}^{N-1}\left(\partial^{\mathrm{M}} E \cap B(x, r)\right)}{r^{N-1}}>0\right\} .
$$

Notice that the relative isoperimetric inequality, together with a continuity argument, easily gives (see (9)) that $\partial^{\mathrm{M}} E \subset \partial^{\mathrm{S}} E$; however (4) guarantees that $\mathcal{H}^{N-1}\left(\partial^{\mathrm{S}} E \backslash \partial^{\mathrm{M}} E\right)=0$, hence $P(E)=\mathcal{H}^{N-1}\left(\partial^{\mathrm{S}} E\right)$ still holds.

With this notation we can prove the following result:

Theorem 8 (Indecomposability and connectedness by arcs). Let $E \subset \mathbb{R}^{2}$ be a set of finite perimeter and let $\left\{E_{i}\right\}_{i \in I}=\mathcal{C C}^{\mathrm{M}}(E)$. Then $\stackrel{\circ}{E}^{\mathrm{M}} \backslash \partial^{\mathrm{S}} E$ is the disjoint union of $\stackrel{\circ}{E}_{i}^{\mathrm{M}} \backslash \partial^{\mathrm{S}} E$ and $x, y \in \stackrel{\circ}{E}^{\mathrm{M}} \backslash \partial^{\mathrm{S}}$ E belong to the same $M$-connected component $E_{i}$ of $E$ if and only if there exists a rectifiable curve $\Gamma$ joining $x$ to $y$ contained in $\stackrel{\circ}{\mathrm{M}}^{\mathrm{M}} \backslash \partial^{\mathrm{S}}$ E. Moreover, for any $\delta>0, \Gamma$ can be chosen so that

$$
\mathcal{H}^{1}(\Gamma) \leq|x-y|+P\left(E_{i}\right)+\delta .
$$

In particular the sets $\stackrel{\circ}{E}_{i}^{\mathrm{M}} \backslash \partial^{\mathrm{S}} E$ are connected.

Our proof of this result actually gives a slightly stronger statement: the sets $\stackrel{\circ}{E}_{i}^{\mathrm{M}} \backslash\left(\partial^{\mathrm{S}} E_{i} \cup L\right)$ are connected by arcs for any $\mathcal{H}^{1}$-negligible set $L \subseteq \mathbb{R}^{2}$; Theorem 8 is a particular case with $L=\ddot{E}_{i}^{\mathrm{M}} \cap \partial^{\mathrm{S}} E$. In order to show this result, our first lemma proves that points in the same $M$-connected component can be joined by curves lying in $\stackrel{\circ}{E}^{\mathrm{M}} \cup \partial^{\mathrm{S}} E$.

Lemma 6. Let $E \subseteq \mathbb{R}^{2}$ be an indecomposable set and let $x, y \in \stackrel{\circ}{E}^{\mathrm{M}} \backslash \partial^{\mathrm{S}} E$. Then there exists a rectifiable curve $\Gamma$ joining $x$ to y contained in ${ }^{\mathrm{M}} \cup \partial^{\mathrm{S}}$ E. Moreover, the curve can be chosen so that $\Gamma \subset \partial^{\mathrm{S}} E \cup L$, where $L$ is the segment joining $x$ to $y$.

Proof. Let $J_{0}$ be the rectifiable Jordan curve corresponding to the simple set $\operatorname{sat}(E)$ and let $J_{i}, 1 \leq i<p$ with $p \in[2, \infty]$, be the rectifiable Jordan curves corresponding to the holes of $E$. Since $x, y \notin \partial^{\mathrm{S}} E$ and $\cup_{i} J_{i} \subset \partial^{\mathrm{M}} E\left(\bmod \mathcal{H}^{1}\right)$, by (27) we obtain that $x$ and $y$ belong to $\operatorname{int}\left(J_{0}\right)$, the topological interior of $J_{0}$, and to $\operatorname{ext}\left(J_{i}\right)$, the topological exterior of $J_{i}$, for $i \geq 1$. If $L$ crosses an hole $\operatorname{int}\left(J_{i}\right)$ we can replace, using Lemma $3, L \cap \overline{\operatorname{int}\left(J_{i}\right)}$ by a curve contained in $J_{i}$, and similarly we can argue if $L$ crosses $\operatorname{ext}\left(J_{0}\right)$. In this way we obtain a rectifiable curve $\Gamma$ fully contained in $\stackrel{\circ}{E}^{\mathrm{M}} \cup \cup_{i \geq 1} J_{i} \subset \stackrel{\circ}{E}^{\mathrm{M}} \cup \partial^{\mathrm{S}} E$.

In order to improve Lemma 6, proving existence of curves contained in ${E^{\mathrm{M}}}^{\mathrm{M}} \backslash$ $\partial^{\mathrm{S}} E$, the natural idea is to enlarge a little bit the holes of $E$ and to shrink a little bit the boundary of $\operatorname{sat}(E)$, to produce a new set whose boundary is "inside" $E$. 
However, this perturbation could not preserve the property that $x$ and $y$ are in the same $M$-connected component, unless we assume that small balls centered at $x$ and $y$ are contained in $E$.

Lemma 7. Let $E \subseteq \mathbb{R}^{2}$ be an indecomposable set, let $x, y \in \mathbb{R}^{2}$ and assume that $B(x, r) \cup B(y, r) \subseteq E\left(\bmod \mathcal{H}^{2}\right)$ for some $r>0$. Then, for any $\mathcal{H}^{1}$-negligible set $N \subseteq \mathbb{R}^{2} \backslash(B(x, r) \cup B(y, r))$ there exists an open set $A \subseteq E$ with finite perimeter such that $N \cup \partial^{\mathrm{S}} E \subseteq A$ and $x, y$ belong to the same $M$-connected component of $E \backslash A$. Moreover, given any $\delta>0$ and any open set $S$ such that $\mathcal{H}^{1}\left(\partial^{\mathrm{M}} E \cap \partial S\right)=0$, we can choose A so that

$$
P(E \backslash A, S) \leq P(E, S)+\delta .
$$

Proof. Assuming with no loss of generality that $r<|x-y|$, we will first build a sequence of open sets $A_{h}$ not intersecting $B(x, r / 2) \cup B(y, r / 2)$, such that $\left|E \cap A_{h}\right| \rightarrow 0, P\left(E \backslash A_{h}\right) \rightarrow P(E)$ and $N \cup \partial^{\mathrm{S}} E \subseteq A_{h}$.

Let $J_{0}, J_{i}$ be as in Lemma 6 and let us denote by $L$ the $\mathcal{H}^{1}$-negligible set $N \cup \partial^{\mathrm{S}} E \backslash \cup_{i} J_{i}$. Given $\epsilon>0$, by (29) we can find $r_{0}<0$ and positive numbers $r_{i}$ such that

$$
\begin{aligned}
& \left|\mathcal{H}^{1}\left(\left\{x \in \mathbb{R}^{2}: \operatorname{sdist}\left(x, J_{0}\right)=r_{0}\right\}\right)-\mathcal{H}^{1}\left(J_{0}\right)\right| \leq \epsilon \\
& \left|\mathcal{H}^{1}\left(\left\{x \in \mathbb{R}^{2}: \operatorname{sdist}\left(x, J_{i}\right)=r_{i}\right\}\right)-\mathcal{H}^{1}\left(J_{i}\right)\right| \leq 2^{-i} \epsilon \quad \forall i \in[1, p) .
\end{aligned}
$$

We also choose balls $B\left(x_{j}, \eta_{j}\right)$ such that their union contains $L$ and $\sum_{j} \eta_{j}<\epsilon$. Choosing $\epsilon=1 / h$, we define

$$
\begin{aligned}
A_{h}:=\left\{x \in \mathbb{R}^{2}: \operatorname{sdist}\left(x, J_{0}\right)>r_{0}\right\} & \cup \bigcup_{1 \leq i<p}\left\{x \in \mathbb{R}^{2}: \operatorname{sdist}\left(x, J_{i}\right)<r_{i}\right\} \\
& \cup \bigcup_{j=1}^{\infty} B\left(x_{j}, \eta_{j}\right) .
\end{aligned}
$$

By construction $A_{h}$ contains $\partial^{\mathrm{S}} E$ and does not intersect $B(x, r / 2) \cup B(y, r / 2)$ for $h$ large enough. Moreover, since $\sum_{j} \pi \eta_{j}^{2} \leq \pi \epsilon^{2}$ and

$$
E \cap A_{h} \subseteq \bigcup_{0 \leq i<p}\left\{x \in \mathbb{R}^{2}:\left|\operatorname{sdist}\left(x, J_{i}\right)\right| \leq\left|r_{i}\right|\right\} \cup \bigcup_{j=0}^{\infty} B\left(x_{j}, \eta_{j}\right)
$$

choosing smaller $r_{i}$ if necessary (again, this is possible due to (29)) we obtain that $\left|E \cap A_{h}\right| \rightarrow 0$. In order to prove that $P\left(E \backslash A_{h}\right)$ converge to $P(E)$ it suffices, by the lower semicontinuity of perimeter, to estimate $P\left(E \backslash A_{h}\right)$ from above. Since $\partial^{\mathrm{M}}\left(E \backslash A_{h}\right) \subset \partial^{\mathrm{M}} E \cup \partial^{\mathrm{M}} A_{h}$ and $\partial^{\mathrm{M}} E \subset \partial^{\mathrm{S}} E \subseteq A_{h}$ we obtain

$$
\begin{aligned}
P\left(E \backslash A_{h}\right) & \leq \mathcal{H}^{1}\left(\partial^{\mathrm{M}} A_{h}\right)=P\left(A_{h}\right) \\
& \leq \sum_{0 \leq i<p} \mathcal{H}^{1}\left(\left\{x \in \mathbb{R}^{2}: \operatorname{sdist}\left(x, J_{i}\right)=r_{i}\right\}\right)+\sum_{j=0}^{\infty} 2 \pi \eta_{j} \\
& \leq \sum_{0 \leq i<p} \mathcal{H}^{1}\left(J_{i}\right)+\frac{2 \pi+2}{h}=P(E)+\frac{2 \pi+2}{h}
\end{aligned}
$$


Now we claim that for $h$ large enough both $x$ and $y$ belong to the same $M$ connected component of $E \backslash A_{h}$; indeed, if this is not true we can find partitions $\left(A_{h}^{1}, A_{h}^{2}\right.$ ) of $E \backslash A_{h}$ (union of suitable $M$-connected components of $E \backslash A_{h}$, see (10)) such that $B(x, r / 2) \subseteq A_{h}^{1}, B(y, r / 2) \subseteq A_{h}^{2}, P\left(E \backslash A_{h}\right) \geq P\left(A_{h}^{1}\right)+P\left(A_{h}^{2}\right)$ and $\left|A_{h}^{1} \cap A_{h}^{2}\right|=0$. Possibly passing to a subsequence, we can assume that $A_{h}^{i}$ locally converge in measure to disjoint sets $A^{i}$ whose union is $E$; the lower semicontinuity of perimeter gives

$$
P(E) \geq P\left(A^{1}\right)+P\left(A^{2}\right)
$$

and, since both $A^{1}$ and $A^{2}$ contain a ball and $E$ is indecomposable, this gives a contradiction.

The final claim follows noticing that the convergence of perimeters implies that $P\left(E \backslash A_{h}, S\right)$ converge to $P(E \backslash A, S)$ as $h \rightarrow \infty$ (see for instance [29], Appendix A).

Finally, we need the following lemma, showing that many circles centered at points in $\stackrel{\circ}{E}^{\mathrm{M}} \backslash \partial^{\mathrm{S}} E$ are fully contained in $\stackrel{\circ}{E}^{\mathrm{M}}$.

Lemma 8. Let $E$ be a set of finite perimeter, let $x \in \stackrel{\circ}{E}^{\mathrm{M}} \backslash \partial^{\mathrm{S}} E$ and define

$$
R:=\left\{t>0: \partial B(x, t) \subset \stackrel{\circ}{E}^{\mathrm{M}}\right\} .
$$

Then $|R \cap(0, r)| / r$ tends to 1 as $r \rightarrow 0^{+}$.

Proof. Let us define $\phi$ equal to 1 on $\stackrel{\circ}{E}^{\mathrm{M}}$, equal to $1 / 2$ on $E^{1 / 2}$ and equal to 0 on ${\overparen{\mathbb{R}^{2} \backslash E}}^{\mathrm{M}}$. Notice that $\phi$ is undefined only on the $\mathcal{H}^{1}$-negligible set $\partial^{\mathrm{M}} E \backslash E^{1 / 2}$, and hence is everywhere defined on almost every circle $\partial B(x, t)$.

Since $x \in \stackrel{\circ}{E}^{\mathrm{M}}$, a simple application of Fubini theorem shows that the set

$$
R_{1}:=\left\{t>0: \mathcal{H}^{1}\left(\partial B(x, t) \cap \stackrel{\circ}{E}^{\mathrm{M}}\right)>0\right\}
$$

satisfies $\left|R_{1} \cap(0, r)\right| / r \rightarrow 1$ as $r \rightarrow 0^{+}$.

Let $\phi_{t}(\theta)=\phi\left(x_{1}+t \cos \theta, x_{2}+t \sin \theta\right)$ and let $\operatorname{Var}\left(\phi_{t}\right)$ be its pointwise variation. The statement would be proved if we show that also the set

$$
R_{2}:=\left\{t>0: \operatorname{Var}\left(\phi_{t}\right)=0\right\}
$$

satisfies $\left|R_{2} \cap(0, r)\right| / r \rightarrow 1$ as $r \rightarrow 0^{+}$, because any $t \in R_{1} \cap R_{2}$ belongs to $R$. To this aim, notice that $\operatorname{Var}\left(\phi_{t}\right) \geq 1 / 2$ for any $t \in(0, \infty) \backslash R_{2}$, hence the density property of $R_{2}$ follows by the inequality

$$
\frac{1}{2}\left|(0, r) \backslash R_{2}\right| \leq \int_{0}^{r} \operatorname{Var}\left(\phi_{t}\right) d t
$$

if we prove that $\int_{0}^{r} \operatorname{Var}\left(\phi_{t}\right) d t / r$ is infinitesimal as $r \rightarrow 0^{+}$. Eventually, this fact follows by the assumption that $x \notin \partial^{\mathrm{S}} E$ and the inequality

$$
\int_{0}^{r} \operatorname{Var}\left(\phi_{t}\right) d t \leq \mathcal{H}^{1}\left(\partial^{\mathrm{M}} E \cap \bar{B}(x, r)\right) \quad \forall r>0 .
$$


In order to prove (31) we first notice that a polar change of coordinates gives

$$
\int_{0}^{r} \operatorname{Var}\left(\varphi_{t}\right) d t=\int_{0}^{r} \int_{0}^{2 \pi}\left|\frac{\partial \varphi_{t}}{\partial \theta}\right| d \theta d t \leq \int_{B(x, r)}|\nabla \varphi| d y
$$

for any $\varphi \in C^{\infty}\left(\mathbb{R}^{\mathrm{N}}\right)$. Now we choose a radial convolution kernel $\rho$ and apply the identity above to the mollified functions $\varphi_{\epsilon}=\phi * \rho_{\epsilon}$; taking into account that $\varphi_{\epsilon}$ pointwise converge to $\phi$ in its domain (see for instance Theorem 4.5.9(24) in [24]), the lower semicontinuity of the variation under pointwise convergence and the inequality (see for instance Proposition 1.15 in [29])

$$
\limsup _{\epsilon \rightarrow 0^{+}} \int_{B(x, r)}\left|\nabla \varphi_{\epsilon}\right| d x \leq P(E, \bar{B}(x, r))=\mathcal{H}^{1}\left(\partial^{\mathrm{M}} E \cap \bar{B}(x, r)\right)
$$

we obtain

$$
\begin{aligned}
\int_{0}^{r} \operatorname{Var}\left(\phi_{t}\right) d t & \leq \int_{0}^{r} \liminf _{\epsilon \rightarrow 0^{+}} \operatorname{Var}\left(\varphi_{\epsilon t}\right) d t \leq \liminf _{\epsilon \rightarrow 0^{+}} \int_{0}^{r} \operatorname{Var}\left(\varphi_{\epsilon t}\right) d t \\
& \leq \liminf _{\epsilon \rightarrow 0^{+}} \int_{B(x, r)}\left|\nabla \varphi_{\epsilon}\right| d y \leq \mathcal{H}^{1}\left(\partial^{\mathrm{M}} E \cap \bar{B}(x, r)\right) .
\end{aligned}
$$

This proves (31) and the lemma.

Proof of Theorem 8. We have proved in (9) that any $x \in \stackrel{\circ}{E}^{\mathrm{M}} \backslash \partial^{\mathrm{S}} E$ is a point of density 1 for some set $E_{i}$.

Let now $x \in \stackrel{\circ}{E}_{i}^{\mathrm{M}} \backslash \partial^{\mathrm{S}} E, y \in \stackrel{\circ}{E}_{j}^{\mathrm{M}} \backslash \partial^{\mathrm{S}} E$, with $i \neq j$. By (27) we obtain that $x$ does not belong neither to the Jordan curve $J_{0}$ corresponding to $\operatorname{sat}\left(E_{i}\right)$ nor to the Jordan curves $J_{k}$ corresponding to the holes of $E_{i}$, and the same holds for $y$. Hence, if $\operatorname{sat}\left(E_{i}\right)$ and $\operatorname{sat}\left(E_{j}\right)$ are disjoint, we conclude that $x \in \operatorname{int}\left(J_{0}\right)$ and $y \in \operatorname{ext}\left(J_{0}\right)$, so that they cannot be connected by a continuous curve not intersecting $J_{0} \subset \partial^{\mathrm{S}} E$. If $\operatorname{sat}\left(E_{j}\right) \subset \operatorname{sat}\left(E_{i}\right)$ then $E_{j}$ is contained in some hole of $E_{i}$ and the same argument applies for some curve $J_{k}$. If $\operatorname{sat}\left(E_{i}\right) \subset \operatorname{sat}\left(E_{j}\right)$ the argument is similar, reversing the roles of $i$ and $j$.

Conversely, given a $M$-connected component $E_{i}$ of $E$, we will prove that any pair of points $x, y \in \stackrel{\circ}{E}_{i}^{\mathrm{M}} \backslash \partial^{\mathrm{S}} E$ can be connected by a rectifiable curve contained in $\stackrel{\circ}{E}_{i}^{\mathrm{M}} \cup \partial^{\mathrm{S}} E$. To this aim, we first choose, according to Lemma 8, strictly decreasing sequences of positive numbers $\eta_{h}, \gamma_{h}$ such that $\partial B\left(x, \eta_{h}\right) \cup \partial B\left(y, \gamma_{h}\right) \subset \stackrel{\circ}{E}_{i}^{\mathrm{M}} \backslash \partial^{\mathrm{S}} E$ (recall that $\stackrel{\circ}{E}_{i}^{\mathrm{M}} \cap \partial^{\mathrm{S}} E$ is $\mathcal{H}^{1}$-negligible), and $2 \pi \sum_{h}\left(\eta_{h}+\gamma_{h}\right)<\delta / 2$. For any integer $h \geq 1$ we define

$$
S_{h}:=\left[B\left(x, \eta_{h-1}\right) \backslash \bar{B}\left(x, \eta_{h}\right)\right] \cup\left[B\left(y, \gamma_{h-1}\right) \backslash \bar{B}\left(y, \gamma_{h}\right)\right],
$$

and

$$
S_{0}=\mathbb{R}^{2} \backslash\left(B\left(x, \eta_{0}\right) \cup B\left(x, \gamma_{0}\right)\right) .
$$

Setting $F_{h}=E_{i} \cup B\left(x, \eta_{h}\right) \cup B\left(y, \gamma_{h}\right)$, the sets $F_{h}$ are still indecomposable (see Proposition 5(i)), hence we can apply Lemma 7 with $N_{h}=\left(\partial^{\mathrm{S}} E \cap \stackrel{\circ}{E}_{i}^{\mathrm{M}}\right) \backslash$ $\left(B\left(x, \eta_{h}\right) \cup B\left(y, \gamma_{h}\right)\right)$ to obtain open sets $A_{h} \supset N_{h} \cup \partial^{\mathrm{S}} F_{h}$ such that $x, y$ belong 
to the same $M$-connected component $G_{h}$ of $F_{h} \backslash A_{h}$. Moreover, since $\partial S_{h} \subset \stackrel{\circ}{F}_{h}^{\mathrm{M}}$, we can also assume that

$$
P\left(F_{h} \backslash A_{h}, S_{h}\right)<P\left(F_{h}, S_{h}\right)+2^{-h-2} \delta .
$$

Finally, we can apply Lemma 6 to $G_{h}$ to obtain a rectifiable curve $\Gamma_{h}$ joining $x$ to $y$, contained in $\stackrel{G}{h}_{h}^{\mathrm{M}} \cup \partial^{\mathrm{S}} G_{h}$ and also in $L \cup \partial^{\mathrm{S}} G_{h}$, where $L$ is the segment joining $x$ to $y$. Since $\partial^{\mathrm{S}} G_{h} \subset \partial^{\mathrm{S}}\left(F_{h} \backslash A_{h}\right)$ we have

$$
\Gamma_{h} \cap S_{h} \subset\left(L \cap S_{h}\right) \cup\left(\partial^{S} G_{h} \cap S_{h}\right) \subset\left(L \cap S_{h}\right) \cup\left(\partial^{S}\left(F_{h} \backslash A_{h}\right) \cap S_{h}\right) .
$$

Since $\partial^{\mathrm{S}} F_{h} \subset \partial^{\mathrm{S}} E_{i}$, using (32), we obtain

$$
\begin{aligned}
\mathcal{H}^{1}\left(\Gamma_{h} \cap S_{h}\right) & \leq \mathcal{H}^{1}\left(\partial^{\mathrm{M}}\left(F_{h} \backslash A_{h}\right) \cap S_{h}\right)+\left(\eta_{h-1}-\eta_{h}\right)+\left(\gamma_{h-1}-\gamma_{h}\right) \\
& \leq \mathcal{H}^{1}\left(\partial^{\mathrm{M}} E_{i} \cap S_{h}\right)+\left(\eta_{h-1}-\eta_{h}\right)+\left(\gamma_{h-1}-\gamma_{h}\right)+2^{-h-2} \delta
\end{aligned}
$$

for any $h \geq 1$. For $h=0$, we have

$$
\begin{aligned}
\mathcal{H}^{1}\left(\Gamma_{0} \cap S_{0}\right) & \leq \mathcal{H}^{1}\left(\partial^{\mathrm{M}}\left(F_{0} \backslash A_{0}\right) \cap S_{0}\right)+\mathcal{H}^{1}\left(L \cap S_{0}\right) \\
& \leq \mathcal{H}^{1}\left(\partial^{\mathrm{M}} E_{i} \cap S_{0}\right)+|x-y|-\left(\eta_{0}+\gamma_{0}\right)+\frac{1}{2^{2}} \delta .
\end{aligned}
$$

Since $\partial^{\mathrm{S}} E \cap \stackrel{\circ}{E}_{i}^{\mathrm{M}} \subseteq A_{h}$ and $\partial^{\mathrm{S}} G_{h} \subset \partial^{\mathrm{S}}\left(F_{h} \backslash A_{h}\right) \subset \mathbb{R}^{2} \backslash A_{h}$, we have $\partial^{\mathrm{S}} G_{h} \subset$ $\mathbb{R}^{2} \backslash\left(\partial^{\mathrm{S}} E \cap \stackrel{\circ}{E}_{i}^{\mathrm{M}}\right)$, and by our choice of $\eta_{h}$ and $\gamma_{h}$ the curves $\Gamma_{h}$ are contained in $\stackrel{\circ}{F}_{h}^{\mathrm{M}} \backslash\left(\partial^{\mathrm{S}} E \cap \stackrel{\circ}{E}_{i}^{\mathrm{M}}\right)$ and hence in $\stackrel{\circ}{E}_{i}^{\mathrm{M}} \backslash \partial^{\mathrm{S}} E$ out of $\bar{B}\left(x, \eta_{h}\right) \cup \bar{B}\left(y, \gamma_{h}\right)$. Using again our choice of $\eta_{h}$ and $\gamma_{h}$ we can build from $\Gamma_{h}$ a locally rectifiable curve $\Gamma$ contained in $\stackrel{\circ}{E}_{i}^{\mathrm{M}} \backslash \partial^{\mathrm{S}} E$ as in Fig. 4 (we have drawn for simplicity the construction only near to $x$ ).

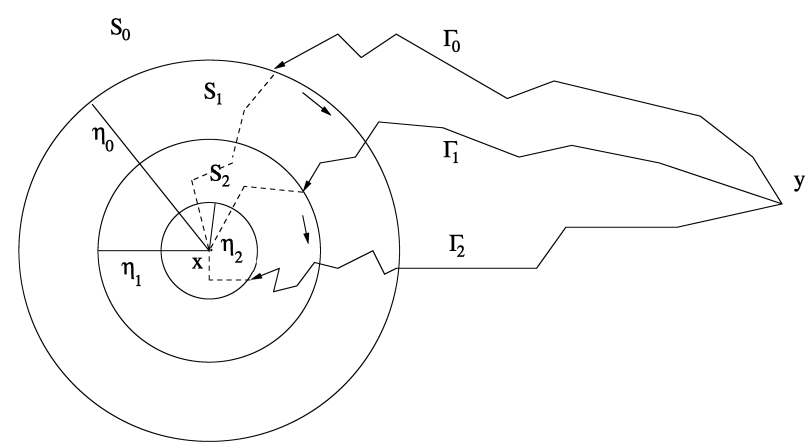

Fig. 4. Recursive construction of $\Gamma$ near to $x$

The estimate on $\mathcal{H}^{1}(\Gamma)$ follows by (33), (34) and by the inclusion

$$
\Gamma \backslash\{x, y\} \subset \bigcup_{h=0}^{\infty}\left(\Gamma_{h} \cap S_{h}\right) \cup \bigcup_{h=0}^{\infty} \partial B\left(x, \eta_{h}\right) \cup \partial B\left(y, \gamma_{h}\right) .
$$




\section{Connected operators for image denoising}

We call "connected operator" any contrast-invariant operator acting on the connected components of level sets. These operators could be defined on BV but we actually do not need neither the finiteness of the total variation nor the summability property. We need only to know that almost every level set has finite perimeter, so that its $M$-connected components can be defined. We therefore introduce a new space of functions that we shall call functions of weakly bounded variation.

Definition 7. We say that a Borel function $u: \Omega \rightarrow[-\infty,+\infty]$ has weakly bounded variation in $\Omega$ if

$$
P(\{u>t\}, \Omega)<\infty \quad \text { for a.e. } t \in \mathbb{R} .
$$

The space of such functions will be denoted by $\mathrm{WBV}(\Omega)$. We call total variation of $u$ and denote by $|D u|$ the measure defined on every Borel subset $B \subseteq \Omega$ as

$$
|D u|(B):=\int_{-\infty}^{+\infty} P(\{u>t\}, B) d t .
$$

It follows from the properties of the perimeter that $|D u|$ is a $\sigma$-additive measure on $\mathcal{B}(\Omega)$. Remark that, by Lemma $1, \operatorname{BV}(\Omega) \subseteq \operatorname{GBV}(\Omega) \subseteq \mathrm{WBV}(\Omega)$ as soon as $\Omega$ is bounded. Furthermore, if $\Omega$ is bounded, connected and with Lipschitz boundary, $u \in \operatorname{WBV}(\Omega)$ and $|D u|(\Omega)<\infty$ then, by Lemma $1, u \in \mathrm{BV}(\Omega)$ and, by the coarea formula, $|D u|$ coincides with the total variation of $u$.

It must be emphasized that WBV is a lattice (because sets of finite perimeter are closed under union and intersection) but is not a vector space. Take indeed the two functions $u(x)=1 / x$ and $v(x)=1 / x-\sin (1 / x)$ defined on $(-1,1)$. Then, clearly, $u, v \in \mathrm{WBV}(-1,1)$ whereas $u-v \notin \mathrm{WBV}(-1,1) \operatorname{since} \sin (1 / x)$ assumes infinitely many times any value $t \in[-1,1]$. However, a strong motivation for the introduction of $\mathrm{WBV}(\Omega)$ is the following result, showing that $\mathrm{WBV}(\Omega)$ is the smallest space containing $\mathrm{BV}(\Omega)$ and invariant under any continuous and strictly increasing contrast change; notice that, by Vol'pert chain rule for distributional derivatives, $\mathrm{BV}(\Omega)$ is stable only under Lipschitz contrast changes.

Theorem 9. Assume that $\Omega$ is bounded, connected and with Lipschitz boundary. For any $u \in \mathrm{WBV}(\Omega)$ there exists a bounded, continuous and strictly increasing function $\phi:[-\infty,+\infty] \rightarrow \mathbb{R}$ such that $\phi \circ u \in \mathrm{BV}(\Omega)$.

Proof. Let $\phi$ be the primitive of $\exp \left(-s^{2}\right) /\left(1+m_{u}(s)\right)$ such that $\phi(-\infty)=0$. Then, since $\phi \circ u$ is bounded and takes its values in $[0, \phi(+\infty)]$,

$$
\begin{aligned}
\int_{-\infty}^{+\infty} m_{\phi \circ u}(t) d t=\int_{0}^{\phi(+\infty)} m_{\phi \circ u}(t) d t & =\int_{-\infty}^{+\infty} m_{u}(s) \phi^{\prime}(s) d s \\
& \leq \int_{-\infty}^{+\infty} \exp \left(-s^{2}\right) d s<\infty
\end{aligned}
$$

hence $\phi \circ u \in \operatorname{BV}(\Omega)$ by Lemma 1(ii). 
Notice that Theorem 9 could be used to extend to $\operatorname{WBV}(\Omega)$ many results of Sect. 3, as for instance the existence of the approximate differential $\nabla u$, the rectifiability of the approximate discontinuity set $S_{u}$, the fact that $\mathcal{H}^{N-1}$-a.e. $x \in S_{u}$ is an approximate jump point, the structure of $D u$ and so on. However, this analysis goes beyond the main goals of this paper and it will not be pursued here.

The space $\operatorname{WBV}(\Omega)$ can be endowed with the following distance (identifying as usual the functions which coincide almost everywhere in $\Omega$ ):

$$
\begin{aligned}
d\left(u_{1}, u_{2}\right):=\int_{\mathbb{R}} e^{-t^{2}} \mid \arctan m_{u_{1}} & -\arctan m_{u_{2}} \mid d t \\
& +\int_{\Omega} e^{-|x|^{2}}\left|\arctan u_{1}-\arctan u_{2}\right| d x .
\end{aligned}
$$

Since $\arctan$ is a homeomorphism between $[-\infty,+\infty]$ and $[-\pi / 2, \pi / 2]$, it is easy to prove that the convergence with respect to $d$ is equivalent to local convergence in measure of both $u$ and $m_{u}$, hence $(\operatorname{WBV}(\Omega), d)$ is a complete metric space.

\section{Vincent's filters}

Luc Vincent introduced in [64] a class of connected operators for denoising an image corrupted by a noise that creates small spots, like for instance impulse noise. Our motivation for the study of such filters is, in addition to the fact that they may be considered as the reference connected operators, their great ability to remove impulse noise. The key idea is to remove connected components of level sets whose Lebesgue measure does not exceed some threshold $\theta$. Luc Vincent defined his filters as operators acting on the space of upper semicontinuous functions, in the framework of Mathematical Morphology. We shall now propose a definition adapted to the space WBV which involves the notion of $M$-connected components. We shall derive new properties of Vincent's filters, regarding in particular the behavior of the total variation. In addition, we shall prove that these filters map SBV onto SBV, Sobolev spaces onto Sobolev spaces and Lipschitz functions onto Lipschitz functions.

First remark that we shall from now assume $\Omega$ bounded with Lipschitz boundary. This is motivated by the fact that an image is generally given on a bounded domain. However, all the definitions and results stated above remain valid since any set $E \subset \Omega$ of finite perimeter in $\Omega$ has finite perimeter in $\mathbb{R}^{\mathrm{N}}$ (see for instance Remark 2.14 in [29]). For the sake of simplicity, we shall write $\partial^{\mathrm{M}} E$ instead of $\partial^{\mathrm{M}} E \cap \Omega$. We start now by defining the action of Vincent's filters on sets of finite perimeter.

Definition 8. Let $E \subset \Omega$ be a set of finite perimeter in $\Omega$ and $\theta \geq 0$. We define $\mathrm{T}_{\theta} E$ as the union of the $M$-connected components $E_{i}$ of $E$ such that $\left|E_{i}\right|>\theta$.

Note that $T_{0} E=E$ and that $\mathrm{T}_{\theta} E$ is well defined up to Lebesgue negligible sets. Moreover, by Proposition 3, it follows that

$$
P\left(\mathrm{~T}_{\theta} E, \Omega\right) \leq P(E, \Omega)
$$

with equality only if $\mathrm{T}_{\theta} E=E\left(\bmod \mathcal{H}^{N}\right)$. 
Proposition 10. Let $E, F \subset \Omega$ be two sets of finite perimeter in $\Omega$. If $E \subseteq F$ $\left(\bmod \mathcal{H}^{N}\right)$, then $\mathrm{T}_{\theta} E \subseteq \mathrm{T}_{\theta} F\left(\bmod \mathcal{H}^{N}\right)$.

Proof. If $E_{i}$ is a $M$-connected component of $E$ with $\left|E_{i}\right|>\theta$, then by Theorem 1 there is a $M$-connected component $F_{j}$ of $F$ such that $E_{i} \subseteq F_{j}\left(\bmod H^{N}\right)$. Since $\left|F_{j}\right|>\theta$, we conclude that $\mathrm{T}_{\theta} E \subseteq \mathrm{T}_{\theta} F\left(\bmod \mathcal{H}^{N}\right)$.

Now we want to extend $\mathrm{T}_{\theta}$ to WBV functions; to this aim, the following lemma will be useful.

Lemma 9. For any monotone family of sets $X_{\lambda}, \lambda \in \mathbb{R}$, there exists a countable set $D \subseteq \mathbb{R}$ such that

$$
\lim _{\mu \rightarrow \lambda} X_{\mu}=X_{\lambda} \quad \text { for all } \lambda \in \mathbb{R} \backslash D,
$$

where convergence means convergence with respect to the finite measure $\mu=$ $e^{-|x|^{2}} \mathcal{L}^{N}$ (or, equivalently, local convergence in measure in $\mathbb{R}^{\mathrm{N}}$ ).

Proof. First remark that the map $\lambda \rightarrow \mu\left(X_{\lambda}\right)$ is real-valued since $\mu(\Omega)=$ $\int_{\Omega} e^{-|x|^{2}} d x<\infty$. Then it is enough to note that this map is monotone, thus has at most countably many discontinuity points, and to choose $D$ as the set of those discontinuity points. We call $D$ the set of discontinuity points of $X_{\lambda}$.

Theorem 10. Let $u \in \operatorname{WBV}(\Omega)$ and $\theta \geq 0$. Then there exists a function $\mathrm{S}_{\theta} u \in$ $\mathrm{WBV}(\Omega)\left(\right.$ resp. $\left.\mathrm{I}_{\theta} u \in \mathrm{WBV}(\Omega)\right)$ such that

$$
\left\{\mathrm{S}_{\theta} u>\lambda\right\}=\mathrm{T}_{\theta}\{u>\lambda\} \quad\left(\text { resp. }\left\{\mathrm{I}_{\theta} u<\lambda\right\}=\mathrm{T}_{\theta}\{u<\lambda\}\right) \quad\left(\bmod H^{N}\right)
$$

with at most countably many exceptions. Any other measurable function $v$ with the same property coincides with $\mathrm{S}_{\theta} u\left(r e s p . \mathrm{I}_{\theta} u\right)$ almost everywhere in $\Omega$. In addition,

$$
\left|D \mathrm{~S}_{\theta} u\right|(B) \leq|D u|(B) \quad \text { and } \quad\left|D \mathrm{I}_{\theta} u\right|(B) \leq|D u|(B) \quad \text { for any Borel set } B \subset \Omega
$$

Proof. Let $X_{\lambda}=\{u>\lambda\}$. By definition of WBV, for almost every $\lambda \in \mathbb{R}, X_{\lambda}$ has finite perimeter and we can define $Y_{\lambda}=\mathrm{T}_{\theta} X_{\lambda}$. Since $\lambda<\lambda^{\prime}$ implies that $X_{\lambda} \supseteq X_{\lambda^{\prime}}$, we infer from Proposition 10 that $\left(Y_{\lambda}\right)$ is a decreasing family. Let $D$ be the set of discontinuity points of $Y_{\lambda}$. Let $D^{*} \subseteq \mathbb{R}$ be countable and dense and define

$$
\mathrm{S}_{\theta} u(x)=\sup \left\{\lambda \in D^{*}: x \in Y_{\lambda}\right\}
$$

We now prove that $\left\{\mathrm{S}_{\theta} u>\lambda\right\}=Y_{\lambda}\left(\bmod H^{N}\right)$ for any $\lambda \notin D$. In fact, we clearly have

$$
Y_{\eta} \subseteq\left\{\mathrm{S}_{\theta} u>\lambda\right\} \subseteq Y_{\rho}
$$

for any $\eta, \rho \in D^{*}, \rho<\lambda<\eta$. If we choose sequences $\eta_{k} \rightarrow \lambda$ and $\rho_{k} \rightarrow \lambda$ in $D^{*}$, Lemma 9 proves that $Y_{\lambda}$ coincides with $\left\{\mathrm{S}_{\theta} u>\lambda\right\}\left(\bmod H^{N}\right)$. In particular, 
$\left\{\mathrm{S}_{\theta} u>\lambda\right\}$ is measurable for any $\lambda \notin D$. By approximation, the same is true for any $\lambda \in \mathbb{R}$. Hence, $\mathrm{S}_{\theta} u$ is measurable.

The uniqueness of $S_{\theta} u$ can be proved by checking, with a similar argument, that if $u_{1}, u_{2}$ are two measurable functions such that $\left\{u_{1}>\lambda\right\}=\left\{u_{2}>\lambda\right\}\left(\bmod H^{N}\right)$ for a dense set of $\lambda$, then $u_{1}=u_{2}$ almost everywhere in $\Omega$.

Remark now that, by assumption, $\{u>\lambda\}$ is a set of finite perimeter in $\Omega$ for almost every $\lambda \in \mathbb{R}$, thus $P(\{u>\lambda\}, B)<+\infty$ for any Borel set $B \subseteq \Omega$. Since $\mathcal{C C}^{\mathrm{M}}\left\{\mathrm{S}_{\theta} u>\lambda\right\} \subseteq \mathcal{C C}^{\mathrm{M}}\{u>\lambda\}$ we deduce by Proposition 3 that $\partial^{\mathrm{M}}\left\{\mathrm{S}_{\theta} u>\lambda\right\} \subseteq$ $\partial^{\mathrm{M}}\{u>\lambda\}\left(\bmod \mathcal{H}^{N-1}\right)$. Recalling that $P(E, B)=\mathcal{H}^{N-1}\left(B \cap \partial^{\mathrm{M}} E\right)$ whenever $E$ has finite perimeter in $B$, it follows that $P\left(\left\{\mathrm{~S}_{\theta} u>\lambda\right\}, B\right) \leq P(\{u>\lambda\}, B)<\infty$ for every Borel subset $B \subseteq \Omega$ and for almost every $\lambda \in \mathbb{R}$. Thus $S_{\theta} u \in \operatorname{WBV}(\Omega)$ and $\left|D S_{\theta} u\right|(B) \leq|D u|(B)$ for any Borel set $B \subseteq \Omega$.

The proof of the existence and the uniqueness of $\mathrm{I}_{\theta} u$ is analogous to the one for $\mathrm{S}_{\theta} u$, by noting that the sets $X_{\lambda}=\{u<\lambda\}$, hence also $Y_{\lambda}=\mathrm{T}_{\theta} X_{\lambda}$, form an increasing family and defining $\mathrm{I}_{\theta} u(x)=\inf \left\{\lambda \in D^{*}: x \in Y_{\lambda}\right\}$. Remark now that $\{u>\lambda\}=\{-u<-\lambda\}$, thus

$$
\mathrm{S}_{\theta} u=-\mathrm{I}_{\theta}(-u) \quad \text { a.e. in } \Omega .
$$

and it follows that $\mathrm{I}_{\theta} u \in \operatorname{WBV}(\Omega)$ and $\left|D \mathrm{I}_{\theta} u\right|(B) \leq|D u|(B)$ for any Borel set $B \subseteq \Omega$.

Remark 11. Recall that, since $u, \mathrm{~S}_{\theta} u$ and $\mathrm{I}_{\theta} u$ are measurable, it is equivalent in the previous theorem to deal with upper level sets instead of strictly upper level sets for both essentially coincide except for at most countably many exceptions.

Since $\mathrm{T}_{\theta}\{u>\lambda\} \subset\{u>\lambda\}$ and $\mathrm{T}_{\theta}\{u<\lambda\} \subset\{u<\lambda\}$ we infer that $\left\{\mathrm{S}_{\theta} u>\lambda\right\} \subset$ $\{u>\lambda\}$ and $\left\{\mathrm{I}_{\theta} u<\lambda\right\} \subset\{u<\lambda\}$ for almost every $\lambda$, hence

$$
\mathrm{S}_{\theta} u \leq u \leq \mathrm{I}_{\theta} u \quad \text { a.e. in } \Omega \text {. }
$$

In order to study the properties of $\mathrm{S}_{\theta}$ and $\mathrm{I}_{\theta}$ in the classical functions spaces BV and $\mathrm{W}^{1, p}$ the following lemma will be useful.

Lemma 10. Let $u, v \in \mathrm{BV}(\Omega)$ such that $|D u|(B) \leq|D v|(B)$ for every Borel set $B \subset \Omega$. Then

(i) $|\nabla u| \leq|\nabla v|$ a.e. in $\Omega$;

(ii) $S_{u} \subseteq S_{v}\left(\bmod \mathcal{H}^{N-1}\right)$;

(iii) $\left|u^{+}-u^{-}\right| \leq\left|v^{+}-v^{-}\right| \mathcal{H}^{N-1}$-a.e. in $\Omega$;

(iv) $\left|D^{c} u\right| \leq\left|D^{c} v\right|$.

Proof. Recall that $|D u|=|\nabla u| \mathcal{L}^{N}+\left|u^{+}-u^{-}\right| \mathcal{H}^{N-1}\left\llcorner J_{u}+\left|D^{c} u\right|\right.$. More precisely, setting

$$
\begin{aligned}
\mathcal{N}_{u} & :=\left\{x \in \Omega: \lim _{r \downarrow 0} r^{-N}|D u|\left(B_{r}(x)\right)=\infty\right\} \\
\text { and } \Theta_{u} & :=\left\{x \in \Omega: \liminf _{r \downarrow 0} r^{1-N}|D u|\left(B_{r}(x)\right)>0\right\}
\end{aligned}
$$


then (see for instance [5]) $\Theta_{u} \subseteq \mathcal{N}_{u},\left|\mathcal{N}_{u}\right|=0, \Theta_{u}$ is $\sigma$-finite with respect to $\mathcal{H}^{N-1}$ and

$D^{a} u=D u\left\llcorner\left(\Omega \backslash \mathcal{N}_{u}\right), \quad D^{j} u=D u\left\llcorner S_{u}=D u\left\llcorner\Theta_{u}\right.\right.\right.$ and $D^{c} u=D u\left\llcorner\left(\mathcal{N}_{u} \backslash \Theta_{u}\right)\right.$. Let $\mathcal{N}=\mathcal{N}_{u} \cup \mathcal{N}_{v}$. Then $|\mathcal{N}|=0$ and for every Borel set $B \subset \Omega \backslash \mathcal{N},|D u|(B)=$ $\left|D^{a} u\right|(B)$ and $|D v|(B)=\left|D^{a} v\right|(B)$. Therefore

$$
\left|D^{a} u\right|(B)=\int_{B}|\nabla u| d x \leq\left|D^{a} v\right|(B)=\int_{B}|\nabla v| d x
$$

and (i) follows since the inequality is true for every Borel set $B \subset \Omega \backslash \mathcal{N}$.

(ii) Let $B=S_{u} \backslash S_{v}$. Then $|D u|(B) \leq|D v|(B),|B|=0$ and $B \subset \Omega \backslash S_{v}$ is $\sigma$-finite with respect to $\mathcal{H}^{N-1}$ so that $D^{a} v \mid(B)=0$ and $\left|D^{c} v\right|(B)=0$ (see [5]). Thus $|D u|(B)=|D v|(B)=0$ and, therefore,

$$
\int_{B}\left|u^{+}-u^{-}\right| d \mathcal{H}^{N-1}=0 .
$$

Since $\left|u^{+}-u^{-}\right|>0$ on $J_{u}$ and $\mathcal{H}^{N-1}\left(S_{u} \backslash J_{u}\right)=0$ we deduce that $\mathcal{H}^{N-1}(B)=0$ thus

$$
S_{u} \subseteq S_{v} \quad\left(\bmod \mathcal{H}^{N-1}\right)
$$

(iii) For every Borel set $B \subseteq J_{u}$

$$
\int_{B}\left|u^{+}-u^{-}\right| d \mathcal{H}^{N-1} \leq \int_{B}\left|v^{+}-v^{-}\right| d \mathcal{H}^{N-1}
$$

and we deduce that

$$
\left|u^{+}-u^{-}\right| \leq\left|v^{+}-v^{-}\right| \quad \mathcal{H}^{N-1} \text {-a.e. in } J_{u} .
$$

The result follows by simply remarking that $\left|u^{+}-u^{-}\right|=0$ for $\mathcal{H}^{N-1}$-a.e. $x \in$ $\Omega \backslash J_{u}$.

(iv) Let $\tilde{\mathcal{N}}=\mathcal{N}_{u} \backslash\left(\Theta_{u} \cup \Theta_{v}\right)$. Since $\Theta_{v}$ is $\sigma$-finite with respect to $\mathcal{H}^{N-1}$ we deduce that $D^{c} u=D u\llcorner\tilde{\mathcal{N}}$. It is a straightforward consequence of the definitions that $\mathcal{N}_{u} \subset \mathcal{N}_{v}$ thus $D v \mathcal{L} \tilde{\mathcal{N}}=D^{c} v\llcorner\tilde{\mathcal{N}}$. For every Borel subset $B \subset \tilde{\mathcal{N}}$ we get

$$
\left|D^{c} u\right|(B)=|D u|(B) \leq|D v|(B)=\left|D^{c} v\right|(B)
$$

and (iv) follows.

The following proposition is a straightforward consequence of the previous lemma and Theorem 10.

Proposition 11. Let $u \in \mathrm{BV}(\Omega)$ and $\theta \geq 0$. Let $\mathrm{T}_{\theta}$ denote any of the operator $\mathrm{S}_{\theta}$ or $\mathrm{I}_{\theta}$. Then

(i) $\left|\nabla \mathrm{T}_{\theta} u\right| \leq|\nabla u|$ a.e. in $\Omega$;

(ii) $S_{\mathrm{T}_{\theta} u} \subseteq S_{u}\left(\bmod \mathcal{H}^{N-1}\right)$;

(iii) $\left|\mathrm{T}_{\theta} u^{+}-\mathrm{T}_{\theta} u^{-}\right| \leq\left|u^{+}-u^{-}\right| \mathcal{H}^{N-1}$-a.e. in $\Omega$;

(iv) $\left|D^{c} \mathrm{~T}_{\theta} u\right| \leq\left|D^{c} u\right|$. 
Remark 6. An interesting consequence of this proposition is that $\mathrm{S}_{\theta}$ and $\mathrm{I}_{\theta}$ map $\operatorname{SBV}(\Omega)$ onto itself in such a way that the jump set is reduced as well as the "height" of the jumps. It is therefore easily seen that any Sobolev space $\mathrm{W}^{1, p}(\Omega)$, $1 \leq p \leq \infty$, is mapped onto itself by $\mathrm{I}_{\theta}$ and $\mathrm{S}_{\theta}$ with a decay of the gradient norm at almost every point. Analogously, any Lipschitz function is mapped onto a Lipschitz function with the same Lipschitz constant.

Finally, we conclude this section with some additional properties of the filters $\mathrm{S}_{\theta}$ and $\mathrm{I}_{\theta}$.

Proposition 12. Let $\theta \geq 0$. Then $\mathrm{S}_{\theta}, \mathrm{I}_{\theta}, \mathrm{I}_{\theta} \mathrm{S}_{\theta}, \mathrm{S}_{\theta} \mathrm{I}_{\theta}$ are monotone and idempotent operators acting on $\mathrm{WBV}(\Omega)$. Moreover, they are covariant with respect to any real continuous and strictly increasing contrast change.

Proof. The monotonicity of the operators is a simple application of the monotonicity of $\mathrm{T}_{\theta}$ on level sets. Observe that if $E$ is a set of finite perimeter in $\Omega$, then $\mathrm{T}_{\theta}\left(\mathrm{T}_{\theta} E\right)=\mathrm{T}_{\theta} E$. Therefore, if $u \in \mathrm{WBV}(\Omega)$, then, for almost every $\lambda \in \mathbb{R}$, $\{u>\lambda\}$ has finite perimeter in $\Omega$ and we have $\mathrm{T}_{\theta}\left(\mathrm{T}_{\theta}\{u>\lambda\}\right)=\mathrm{T}_{\theta}\{u>\lambda\}$. By the uniqueness property stated in Theorem 10, we deduce that $\mathrm{S}_{\theta}\left(\mathrm{S}_{\theta} u\right)=\mathrm{S}_{\theta} u$ almost everywhere in $\Omega$. Equation (36) implies that $\mathrm{I}_{\theta}$ is idempotent as well. Now, let us prove that

$$
\mathrm{S}_{\theta} \mathrm{I}_{\theta} \mathrm{S}_{\theta} u=\mathrm{I}_{\theta} \mathrm{S}_{\theta} u .
$$

Indeed, let $\lambda \in \mathbb{R}$ be such that $\{u>\lambda\}$ is a set of finite perimeter in $\Omega,\left\{\mathrm{S}_{\theta} u \leq \lambda\right\}=$ $\left\{\mathrm{S}_{\theta} u<\lambda\right\}$, and $\left\{\mathrm{I}_{\theta} \mathrm{S}_{\theta} u \leq \lambda\right\}=\left\{\mathrm{I}_{\theta} \mathrm{S}_{\theta} u<\lambda\right\}\left(\bmod H^{N}\right)$. By Theorem 10, $\left\{\mathrm{S}_{\theta} u>\lambda\right\}$ $=\mathrm{T}_{\theta}\{u>\lambda\},\left\{\mathrm{I}_{\theta} \mathrm{S}_{\theta} u<\lambda\right\}=\mathrm{T}_{\theta}\left\{\mathrm{S}_{\theta} u<\lambda\right\},\left\{\mathrm{S}_{\theta} \mathrm{I}_{\theta} \mathrm{S}_{\theta} u>\lambda\right\}=\mathrm{T}_{\theta}\left\{\mathrm{I}_{\theta} \mathrm{S}_{\theta} u>\lambda\right\}$ $\left(\bmod H^{N}\right)$. Then we prove that

$$
\left\{\mathrm{S}_{\theta} \mathbf{I}_{\theta} \mathrm{S}_{\theta} u>\lambda\right\}=\left\{\mathrm{I}_{\theta} \mathrm{S}_{\theta} u>\lambda\right\} \quad\left(\bmod H^{N}\right) .
$$

Otherwise, there exists a $M$-connected component $Q$ of $\left\{\mathrm{I}_{\theta} \mathrm{S}_{\theta} u>\lambda\right\}$ with $0<$ $|Q| \leq \theta$. Thus $Q$ is a $M$-connected component of $\mathbb{R}^{\mathrm{N}} \backslash\left\{\mathrm{I}_{\theta} \mathrm{S}_{\theta} u \leq \lambda\right\}=\mathbb{R}^{\mathrm{N}} \backslash$ $\left\{\mathrm{I}_{\theta} \mathrm{S}_{\theta} u<\lambda\right\}=\mathbb{R}^{\mathrm{N}} \backslash \mathrm{T}_{\theta}\left\{\mathrm{S}_{\theta}<\lambda\right\}=\mathbb{R}^{\mathrm{N}} \backslash \mathrm{T}_{\theta}\left\{\mathrm{S}_{\theta} \leq \lambda\right\}$ and, according to Theorem 1, we may write

$$
\partial^{M} Q=\cup_{k=1}^{p} \partial^{M} F_{k} \quad\left(\bmod H^{N-1}\right),
$$

where $F_{k}, k=1, \ldots, p$, denote the $M$-connected components of $\mathrm{T}_{\theta}\left\{\mathrm{S}_{\theta} u \leq \lambda\right\}$ such that $\partial^{M} F_{k} \cap \partial^{M} Q \neq \varnothing\left(\bmod H^{N-1}\right)$. In particular, $F_{k}, k=1, \ldots, p$, are $M$-connected components of $\left\{\mathrm{S}_{\theta} u \leq \lambda\right\}$ such that $\left|F_{k}\right|>\theta$. It follows that $Q$ cannot be contained in $\left\{\mathrm{S}_{\theta} u \leq \lambda\right\}$. Hence, $Q$ contains at least a $M$-connected component of $\left\{\mathrm{S}_{\theta} u>\lambda\right\}$ and, therefore, $|Q| \geq \theta$. This contradiction proves (39) and, as a consequence, (38). Since $\mathrm{I}_{\theta}$ is idempotent, we obtain

$$
\mathrm{I}_{\theta} \mathrm{S}_{\theta} \mathrm{I}_{\theta} \mathrm{S}_{\theta} u=\mathrm{I}_{\theta} \mathrm{I}_{\theta} \mathrm{S}_{\theta} u=\mathrm{I}_{\theta} \mathrm{S}_{\theta} u .
$$

Let us prove the covariance of $\mathrm{S}_{\theta}$ with respect to any real continuous increasing contrast change. This is due to the fact the family of level sets is globally invariant by such a contrast change. Let $u \in \operatorname{WBV}(\Omega)$ and let $g: \mathbb{R} \rightarrow \mathbb{R}$ be a real continuous 
increasing function. Then, for almost every $\lambda \in \mathbb{R},\{g(u)>g(\lambda)\}=\{u>\lambda\}$, hence, $\mathrm{T}_{\theta}\{g(u)>g(\lambda)\}=\mathrm{T}_{\theta}\{u>\lambda\}$ and, by definition, $\left\{\mathrm{S}_{\theta} g(u)>g(\lambda)\right\}=$ $\left\{\mathrm{S}_{\theta} u>\lambda\right\}$. Thus $\left\{g^{-1} \mathrm{~S}_{\theta} g(u)>\lambda\right\}=\left\{\mathrm{S}_{\theta} u>\lambda\right\}$. From the uniqueness statement of Theorem 10, we conclude that $\mathrm{S}_{\theta} g(u)=g\left(\mathrm{~S}_{\theta} u\right)$ a.e. in $\Omega$. The corresponding statements for $\mathrm{I}_{\theta}, \mathrm{I}_{\theta} \mathrm{S}_{\theta}, \mathrm{S}_{\theta} \mathrm{I}_{\theta}$ are proved in the same way. The monotonicity assertion is straightforward and we shall omit the details.

\section{Experiments}

First recall that an image can be naturally represented as a piecewise constant function, each pixel being considered as a square with measure one. We have illustrated in Fig. 5 the internal and external boundaries of some level sets of an image (see Sect. 7). For the sake of simplicity, we shall also use the terms topographic map to refer to this representation. It is a straightforward consequence of Theorem 6 and the reconstruction formula $u(x)=\sup \{t: x \in\{u>t\}\}=$ $\inf \{t: x \in\{u<t\}\}$ that the topographic map is a complete and contrast-invariant representation of the image. Remark that, for the sake of readability, we have actually illustrated in Fig. 5 the partial topographic map obtained by taking into account only those level sets separated by at least 10 grey levels.

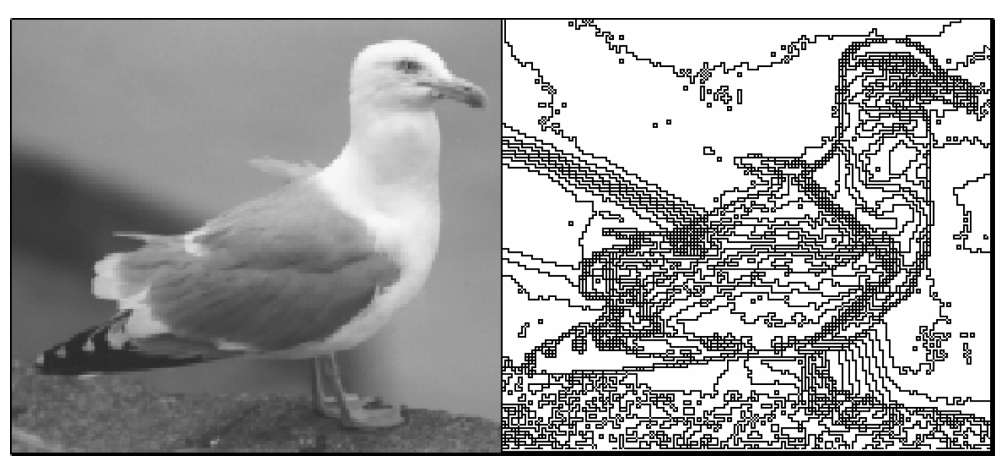

Fig. 5. An image and its partial topographic map (grey level step $=10$ )

Figure 6 illustrates the ability of the Vincent's filter $\mathrm{I}_{\theta} \mathrm{S}_{\theta}$ to remove impulse noise in an image. Recall that impulse noise replaces the value of a prescribed number of pixels, uniformly distributed in the image, by a random value taken between 0 and 255, according to a uniform distribution law. The algorithm for computing the action of $\mathrm{I}_{\theta}$ is the following: let $x_{0}$ be a pixel where the image, denoted by $u$, assumes a local minimum and $\lambda=u\left(x_{0}\right)$. Adding progressively pixels in the neighborhood of $x_{0}$, one can construct the connected component $I(\lambda)$ containing $x_{0}$ of the set $\{x, u(x) \leq \lambda\}$. Then, setting $\lambda:=\lambda+1$, the process is iterated until $|I(\lambda)| \geq \theta$. Finally, each pixel in $I(\lambda)$ is given the value $\lambda$. The whole process is performed for each local minimum of $u$.

The algorithm for $S_{\theta}$ is stricly analogous, starting from a local maximum and computing iteratively the connected component $S(\lambda)$ containing $x_{0}$ of the set 


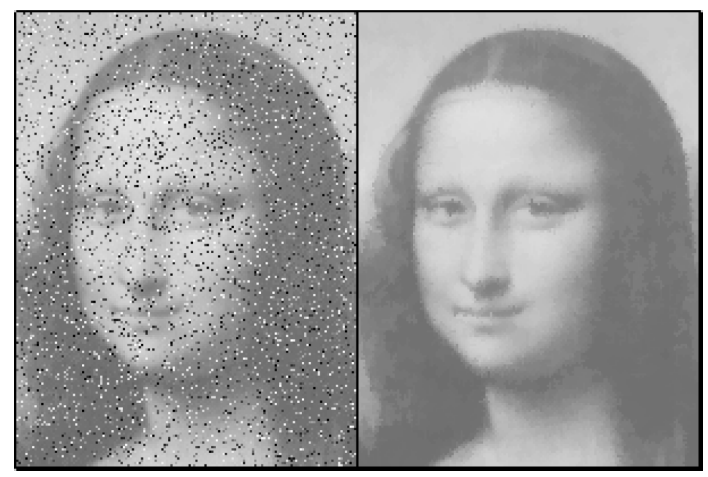

Fig. 6. An image corrupted by an impulse noise with frequency $15 \%$ and the result of the denoising performed by $\mathrm{I}_{10} \mathrm{~S}_{10}$

$\{x, u(x) \geq \lambda\}$, where $\lambda$ is initially given the value $u\left(x_{0}\right)$ and is lowered until $|S(\lambda)| \geq \theta$. Again, each pixel in the ultimate $S(\lambda)$ is given the value $\lambda$.

We shall not address here the problem of the consistency of these algorithms, that is the question whether they converge to the operator $\mathrm{I}_{\theta} \mathrm{S}_{\theta}$ as defined for functions, when the discrete grid tends to the continuous plane. This question is obviously far beyond the scope of this paper.

Three properties of $\mathrm{I}_{\theta} \mathrm{S}_{\theta}$ are particularly relevant in view of an automated denoising: the idempotence, which prevents from caring about the number of iterations, the dependence on a single parameter $\theta$, which makes the filter much easier to handle with and, finally, the ability of $\mathrm{I}_{\theta} \mathrm{S}_{\theta}$ to preserve the unnoisy parts of the image (see Fig. 7) which ensures that only noise is processed.

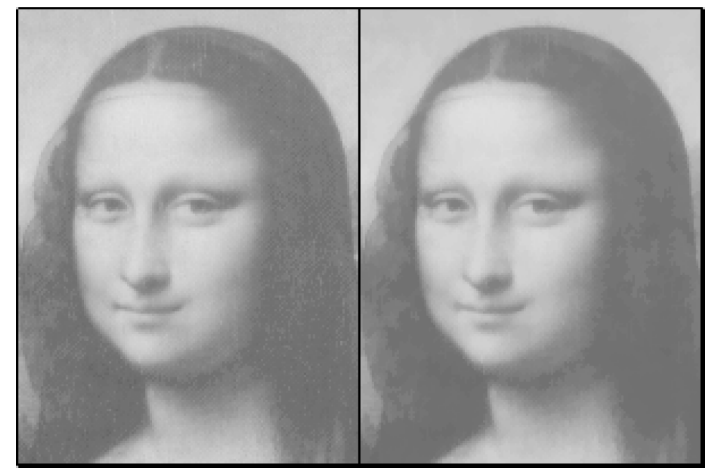

Fig. 7. An uncorrupted image and the result of the filtering by $\mathrm{I}_{10} \mathrm{~S}_{10}$. This experiment illustrates the ability of Vincent's filter to preserve uncorrupted parts of an image

Acknowledgements. We all acknowledge partial support by the TMR European project "Viscosity solutions and their applications", reference FMRX-CT98-0234. The first author acknowledges partial support from the MURST project "Equazioni Differenziali e Calcolo delle Variazioni”, reference 9701226040 . The second, third and fourth authors acknowledge 
partial support of CNRS through a PICS project. The second author acknowledges partial support by EC Project PAVR, reference ERB FMRX-CT96-0036.

\section{References}

1. Alvarez, L., Gousseau, Y., Morel, J.M.: The size of objects in natural and artificial images. Adv. Imaging Electr. Phys. 111 (1999)

2. Alvarez, L., Guichard, F., Lions, P.L., Morel, J.M.: Axioms and fundamental equations of image processing. Arch. Rat. Mech. Anal. 16, 200-257 (1993)

3. Ambrosio, L.: A compactness theorem for a new class of functions of bounded variation. Boll. Un. Mat. Ital. B(7) 3, 857-881 (1989)

4. Ambrosio, L.: Existence theory for a new class of variational problems. Arch. Rat. Mech. Anal. 111, 291-322 (1990)

5. Ambrosio, L., Fusco, N., Pallara, D.: Functions of Bounded Variation and Free Discontinuity Problems. Oxford University Press, 2000

6. Ballester, C., Cubero-Castan, E., Gonzalez, M., Morel, J.M.: Image intersection and applications to satellite imaging. Preprint, C.M.L.A., Ecole Normale Supérieure de Cachan, 1998

7. Brady, M.: Criteria for representation of shape. Chapter in: Human and Machine Vision. Beck et al. eds., New York-Orlando, FL: Academic Press, 1983

8. Brice, C., Fennema, C.: Scene analysis using regions. Artificial Intelligence 1, 205-226 (1970)

9. Canny, J.F.: A computational approach to edge detection. IEEE Trans. Patt. Anal. Machine Intel. 8, 769-798 (1986)

10. Carriero, M., De Giorgi, E., Leaci, A.: Existence theorem for a minimum problem with free discontinuity set. Arch. Rat. Mech. Anal. 108, 195-218 (1989)

11. Caselles, V., Coll, B., Morel, J.M.: A Kanizsa programme. Preprint Ceremade, Univ. Paris-Dauphine, 1995

12. Caselles, V., Coll, B., Morel, J.M.: Topographic maps. Int. J. Comp. Vision (to appear)

13. Congedo, G., Tamanini, I.: Problemi di partizioni ottimali con dati illimitati. Atti Accad. Naz. Lincei Cl. Sci. Fis. Mat. Natur., Rend. Lincei (9) Mat. Appl. 4, 103-108 (1993)

14. Davis, L.: A survey of edge detection techniques. Comput. Graph. Image Proc. 4, 248-270 (1975)

15. De Giorgi, E.: Su una teoria generale della misura $(r-1)$-dimensionale in uno spazio ad $r$ dimensioni. Ann. Mat. Pura Appl., IV. Ser. 36, 191-213 (1954)

16. De Giorgi, E.: Sulla proprietà isoperimetrica dell'ipersfera, nella classe degli insiemi aventi frontiera orientata di misura finita. Atti Accad. Naz. Lincei Mem. Cl. Sci. Fis. Mat. Nat. Sez. I, (8) 5, 33-44 (1958)

17. De Giorgi, E., Ambrosio, L.: Un nuovo tipo di funzionale del Calcolo delle Variazioni. Atti Accad. Naz. Lincei, s. 8 82, 199-210 (1988)

18. De Giorgi, E.: Introduzione ai problemi di discontinuità libera. In: Symmetry in Nature. A volume in honour of Luigi A. Radicati di Brozolo, I, Scuola Norm. Sup. Pisa, 265-285, 1989

19. Donoho, D.L., Johnstone, I.M., Kerkyacharian, G., Picard, D.: Wavelet shrinkage: asymptopia? J. R. Statist. Soc. B 57, 301-369 (1995)

20. Durand, S., Malgouyres, F., Rougé, B.: Image deblurring, spectrum interpolation and application to satellite imaging. Submitted to Math. Model. Num. Anal., 1999

21. Dolzmann, G., Müller, S.: Microstructures with finite surface energy: the two-well problem. Arch. Rat. Mech. Anal. 132, 101-141 (1995)

22. Evans, L.C., Gariepy, R.F.: Measure Theory and Fine Properties of Functions. Studies in Advanced Math., CRC Press 1992

23. Falconer, K.J.: The Geometry of Fractal Sets. Cambridge: Cambridge University Press 1985

24. Federer, H.: Geometric Measure Theory. Berlin, Heidelberg, New York: Springer 1969 
25. Fleming, W.H.: Functions with generalized gradient and generalized surfaces. Ann. Matematica 44, 93-103 (1957)

26. Fleming, W.H.: Functions whose partial derivatives are measures. Ill. J. Math. 4, 452478 (1960)

27. Froment, J.: A functional analysis model for natural images permitting structured compression. COCV 4, 473-495 (1999)

28. Giaquinta, M., Modica, G., Souček, J.: Cartesian currents in the calculus of variations, Part I: Cartesian currents. Berlin, Heidelberg, New York: Springer 1998

29. Giusti, E.: Minimal Surfaces and Functions of Bounded Variation. Basel: Birkhäuser 1994

30. Goffman, C., Waterman, D.: Approximately continuous transformations. Proc. Am. Math. Soc. 12, 116-121 (1961)

31. Guichard, F., Morel, J.M.: Partial differential equations and image iterative filtering. State of the Art in Numerical Analysis. Oxford: Oxford University Press 1997

32. Kanizsa, G.: Organization in Vision. New York: Praeger 1979

33. Kimia, B.B., Tannenbaum, A., Zucker, S.W.: On the evolution of curves via a function of curvature, 1: the classical case. J. Math. Anal. Appl. 163, 438-458 (1992)

34. Koenderink, J.J., van Doorn, A.J.: Dynamic shape. Biol. Cyber. 53, 383-396 (1986)

35. Kuratowski, C.: Topologie I, II. Editions J. Gabay 1992

36. Kirchheim, B.: Lipschitz minimizers of the 3-well problem having gradients of bounded variation (Forthcoming)

37. Lantuéjoul, C., Beucher, S.: On the use of geodesic metric in image analysis. J. Microscopy 121, 39-49 (1981)

38. Lantuéjoul, C., Maisonneuve, F.: Geodesic methods in image analysis. Pattern Recognition 17, 117-187 (1984)

39. Leonardi, G.P.: Optimal subdivisions of $n$-dimensional domains. PhD Thesis, Università di Trento, 1998

40. Lowe, D.G.: Organization of smooth image curves at multiple scales. Int. J. Comput. Vision 3, 119-130 (1989)

41. Mackworth, A., Mokhtarian, F.: Scale-Based description and recognition of planar curves and two-dimensional shapes. IEEE Trans. Patt. Anal. Machine Intel. 8, 1986

42. Marr, D.: Vision. Freeman and Co. 1981

43. Marr, D., Hildreth, E.: Theory of edge detection. Proc. R. Soc. London B 207, 187-217 (1980)

44. Masnou, S.: Filtrage et désocclusion d'images par méthodes d'ensembles de niveau, $\mathrm{PhD}$ Thesis, Ceremade, Université Paris-Dauphine 1998

45. Matheron, G.: Random sets and integral geometry. New York: John Wiley and Sons 1975

46. Mattila, P.: Geometry of Sets and Measures in Euclidean Spaces. Cambridge Studies in Advanced Mathematics. Cambridge: Cambridge University Press 1995

47. Meyer, F., Beucher, S.: Morphological Segmentation. J. Visual Commun. Image Representation 1, 21-46 (1990)

48. Monasse, P.: Contrast invariant image registration. Proc. of the International Conf. on Acoustics, Speech and Signal Processing, Phoenix, Arizona, 6, 3221-3224 (1999)

49. Monasse, P., Guichard, F.: Fast computation of a contrast invariant image representation. IEEE Trans. Image Proc. (to appear 1999)

50. Montaneri, G.H.: On the optimal detection of curves in noisy pictures. Comm. Assoc. Comput. Mach. 14, 335-345 (1971)

51. Morel, J.M., Solimini, S.: Variational Methods in Image Processing. Basel: Birkhäuser 1994

52. Mumford, D., Shah, J.: Optimal approximations by piecewise smooth functions and associated variational problems. Commun. Pure Appl. Math. 17, 577-685 (1989)

53. Nitzberg, M., Mumford, D.: The 2.1-D sketch. Preprint, Department of Mathematics. Harvard University Cambridge MA

54. Pallara, D.: Some new results on functions of bounded variation. Rend. Accad. Naz. Sci. XL Mem. Mat. 14, 295-321 (1990) 
55. Rudin, L.I.: Images, numerical analysis of singularities and shock filters. PhD dissertation, Caltech, Pasadena, California no. 5250:TR, 1987

56. Rudin, L.I., Osher, S., Fatemi, E.: Nonlinear total variation based noise removal algorithms. Physica D 60, 259-269

57. Salembier, P.: Morphological multiscale segmentation for image coding. Signal Processing. Special Issue on Nonlinear Signal Processing 38, 359-386 (1994)

58. Salembier, P., Serra, J.: Flat zones filtering, connected operators and filters by reconstruction. IEEE Trans. Image Proc. 4, 1153-1160 (1995)

59. Salembier, P., Brigger, P., Casas, J.R., Pardàs, M.: Morphological operators for image and video compression. IEEE Trans. Image Proc. 5, 881-897 (1996)

60. Serra, J.: Image analysis and mathematical morphology. London: Academic Press 1982

61. Serra, J.: Image analysis and mathematical morphology. Volume 2: Theoretical Advances. London: Academic Press 1988

62. Serra, J., Salembier, P.: Connected operators and pyramids. Proc. SPIE Image Algebra Math. Morphology, San Diego, CA, SPIE 2030, 65-76 (1993)

63. Simon, L.: Lectures on geometric measure theory. Proc. Centre Math. Anal., Australian Nat. Univ. 3, 1983

64. Vincent, L.: Morphological area openings and closings for grey-scale images. Proc. of the Workshop "Shape in Picture", 1992, Driebergen, The Netherlands, Springer-Berlin, 197-208, 1994

65. Vincent, L.: Grey scale area openings and closings, their efficient implementation and applications. Proc. Workshop Mathematical Morphology and Applications to Signal Processing, Barcelona, Spain, May 1993, 22-27

66. Vincent, L., Soille, P.: Watersheds in digital spaces: An efficient algorithm based ion immersion simulations. IEEE Trans. Patt. Anal. Machine Intel. 13, 583-598 (1991)

67. Vol'pert, A.I.: Spaces BV and quasi-linear equations. Math. USSR Sb. 17, 225-267 (1967)

68. Wertheimer, M.: Untersuchungen zur Lehre der Gestalt, II. Psychologische Forschung 4, 301-350 (1923)

69. Weszka, J.S.: A survey of threshold selection techniques. Comput. Graph. Image Proc. 7, 259-265 (1978)

70. Yaroslavsky, L.P., Eden, M.: Fundamentals of digital optics. Boston: Birkhäuser 1996

71. Zhikov, V.V.: Connectedness and homogenization. Examples of fractal conductivity. Mat. Sbornik 187, 1109-1147 (1996)

72. Ziemer, W.P.: Weakly Differentiable Functions. GTM 120, Berlin, Heidelberg, New York: Springer 1989 